No. 21-3

\title{
Who Gets Medication-assisted Treatment for Opioid Use Disorder, and Does It Reduce Overdose Risk? Evidence from the Rhode Island All-payer Claims Database Mary A. Burke, Riley Sullivan, Katherine Carman, Hefei Wen, J. Frank Wharam, and Hao Yu
}

\begin{abstract}
:
This paper uses the all-payer claims database (APCD) for Rhode Island to study three questions about the use of medication-assisted treatment (MAT) for opioid use disorder (OUD): (1) Does MAT reduce the risk of opioid overdose; (2) are there systematic differences in the uptake of MAT by observable patient-level characteristics; and (3) how successful were federal policy changes implemented in 2016 that sought to promote increased use of buprenorphine, one of three medication options within MAT? Regarding the first question, we find that MAT as practiced in Rhode Island is associated with a reduced risk of repeated opioid overdose among patients who had an initial nonfatal opioid overdose, consistent with the strong endorsement of MAT by public health officials. Concerning the second, we find that factors such as age, gender, health insurance payer, and the poverty rate in one's residential Zip code are associated with significant differences in the chance of receiving methadone and/or buprenorphine, suggesting that certain groups may face unwarranted disparities in access to MAT. About the third question, we find that a 2016 federal rule change enabled at least some experienced Rhode Island buprenorphine prescribers to reach more patients, and a separate 2016 policy aimed at recruiting new buprenorphine prescribers was also found to be effective. However, the data also suggest that many more patients in the state could be treated with buprenorphine if prescribers took full advantage of their prescribing limits.
\end{abstract}

JEL Classifications: I18, I13, I14, I12

Keywords: medication-assisted treatment, opioid use disorder, overdose, Rhode Island, all-payer claims database, methadone, buprenorphine

Mary A. Burke is a senior economist and policy advisor in the Federal Reserve Bank of Boston Research Department; her email is MaryBurke@bos.frb.org. Riley Sullivan is a senior policy analyst in the Boston Fed's Research Department. Katherine Carman is a senior economist at the RAND Corporation and a professor at the Pardee RAND Graduate School. Hefei Wen, J. Frank Wharam, and Hao Yu are faculty members of Harvard Medical School and Harvard Pilgrim Health Care Institute.

The authors wish to thank Samuel Makikalli, Morgan Klaeser, David Schramm, Melissa Gentry, and Bret Fontecchio for research assistance and data analysis; Darcy Saas, Delia Sawhney, and Stephanie Zierten for administrative and legal support; and Jones George and Bret Fontecchio for technical support. For helpful comments and feedback, they thank Jeffrey Thompson, Geoff Tootell, and participants in a Federal Reserve Bank of Boston seminar, as well as various staff members at the Brown Policy Lab, the Rhode Island Department of Health, and the Rhode Island Department of Behavioral Health, Developmental Disabilities, and Hospitals.

Data for this analysis were obtained through an approved request to HealthFacts RI, Rhode Island's all-payer claims database, as administered by the Rhode Island Department of Health (RIDOH). Data were obtained for the months April 2011 through May 2019. RIDOH is not responsible for the authors' analysis, opinions, and conclusions contained in this document. The views expressed herein are those of the authors and do not indicate concurrence by the Federal Reserve Bank of Boston, the principals of the Board of Governors, or the Federal Reserve System. This paper presents preliminary analysis and results intended to stimulate discussion and critical comment. This paper, which may be revised, is available on the website of the Federal Reserve Bank of Boston at http://www.bostonfed.org/economic/wp/index.htm. 


\section{Motivation}

The high prevalence of opioid use disorder (OUD) in the United States remains an urgent public health concern. After increasing dramatically in the 2010s, national opioid-related mortality levelled off between 2017 and 2019, but it experienced a resurgence in 2020 amid the COVID19 pandemic. ${ }^{1}$ Since the early 2000s, Rhode Island has ranked among the states experiencing an especially severe and protracted crisis of opioid abuse - see Figure 1. In 2018 the state's opioidrelated overdose death rate was 10th highest in the United States among the 38 states that were ranked by the Centers for Disease Control and Prevention (CDC) ${ }^{2}$ Like many other states, Rhode Island experienced an apparent increase in opioid-related mortality in $2020{ }^{3}$

Medication-assisted treatment (MAT) for opioid use disorder consists of administering any of the US Food and Drug Administration (FDA)-approved medications methadone, buprenorphine, or naltrexone in conjunction with behavioral therapy. MAT is strongly endorsed by the World Health Organization and the United States Department of Health and Human Services, based on a broad body of clinical evidence demonstrating its effectiveness in reducing the abuse of opioids and all-cause mortality. ${ }^{4}$ Despite the strong support for MAT in the medical and public health communities, according to some estimates fewer than 10 percent of US patients with diagnosed OUD receive MAT, ${ }^{5}$ and many OUD sufferers receive no treatment of any kind for their condition (Saloner and Karthikeyan 2015). A variety of factors may inhibit the uptake of MAT, such as regulations that limit the availability of the medications involved, gaps in health

\footnotetext{
${ }^{1}$ More than 40 states have reported increases in opioid-related fatalities in 2020 compared with 2019. See American Medical Association, "Reports of Increases in Opioid- and Other Drug-related Overdose and Other Concerns during COVID Pandemic" December 9, 2020, https://www.ama-assn.org/system/files/2020-12/issue-brief-increases-inopioid-related-overdose.pdf.

${ }^{2}$ Three other New England states - New Hampshire, Massachusetts, and Connecticut—had even higher opioid death rates per capita than Rhode Island in 2018 (Kaiser Family Foundation 2020).

3 This statement is based on Rhode Island data on overdose deaths from all drugs combined. Given previous patterns, opioid-related overdoses likely account for a large majority of those combined deaths. See Rhode Island Department of Health, "Drug Overdose Deaths"; and Edward Fitzpatrick, "Another Pandemic Fallout: Deaths from Accidental Drug Overdoses Are Soaring in Rhode Island," Boston Globe, August 5, 2020.

${ }^{4}$ The medical literature supporting the use of MAT for OUD is vast. See, for example, Connery (2015), Gibson et al. (2008), and Ma et al. (2018). In addition to its benefits for individual OUD patients, MAT has been found to offer broader benefits to public health, such as reductions in HIV and hepatitis C risk behaviors as well as reductions in criminal behavior (Evans et al. 2019). Observational studies from Massachusetts (Larochelle et al. 2018) and Vermont (Mohlman et al. 2016) find, respectively, that MAT is associated with lower risk of fatal opioid overdose and lower health-care expenditures.

${ }^{5}$ See Emma Sandoe, Carrie E. Fry, and Richard Frank, "Policy Levers That States Can Use to Improve Opioid Addiction Treatment and Address the Opioid Epidemic," Health Affairs Blog, October 2, 2018.
} 
insurance coverage, and social stigmas surrounding substance abuse in general and around these medications specifically.

Since 2002 a variety of federal and state policies have aimed to help more OUD patients gain access to MAT. ${ }^{6}$ Rhode Island has adopted notably robust, innovative policies to promote comprehensive care for OUD that includes MAT, and the available evidence suggests that the state has an above-average rate of uptake of $\mathrm{MAT}^{7}$ The fact that opioid-related mortality rates remain elevated, in Rhode Island and on average in the United States, raises questions concerning the success of recent policy measures in achieving sufficient increases in the uptake of MAT and concerning the practical efficacy of MAT in reducing overdose risk. This paper uses the all-payer claims database (APCD) for Rhode Island — known as HealthFacts RI — to study (1) the effectiveness of MAT as practiced in Rhode Island in preventing opioid overdoses, (2) the factors at the patient level that either inhibit or facilitate the uptake of MAT, and (3) the impact on buprenorphine prescribing patterns in Rhode Island of federal policies implemented in 2016 that aimed to expand access to buprenorphine. Rhode Island's high prevalence of opioid use disorder and the data set's broad coverage of the state's population amplify the relevance of our results to policy discussions both within Rhode Island and beyond. Very few previous studies use large administrative data sets to address these questions.

First, we find evidence that MAT as practiced in Rhode Island helps to reduce the risk of opioid overdose, consistent with previous observational studies of patients in Massachusetts (Larochelle et al. 2018) and England (Pierce et al. 2015). Specifically, we show that among patients who had an initial (nonfatal) overdose, those who had received MAT in the preceding three months were significantly less likely to experience a second overdose that may or may not have been fatal, controlling for a variety of potentially confounding factors.

Second, we find that among patients diagnosed with opioid dependence, members of certain groups may face unwarranted disparities in treatment access, even after we control for numerous confounding factors. Women are less likely to receive MAT (either methadone or

\footnotetext{
${ }^{6}$ In New England, Vermont pioneered the "hub and spoke" system for treating OUD, which enabled otherwise underserved rural citizens to access evidence-based treatments for opioid use disorder, including MAT, and Massachusetts also has taken strong actions to promote access to MAT. See Manchester and Sullivan (2019). ${ }^{7}$ See Section II.C for details.
} 
buprenorphine) than men, and individuals with comorbid alcohol use disorder are less likely to be treated with methadone than other patients. Buprenorphine treatment is less likely, but methadone more likely, among individuals residing in Zip codes with elevated poverty rates. Medicaid patients are much more likely to receive methadone, but not more likely to be treated with buprenorphine, than non-Medicaid patients. Patients in older age groups - especially those ages 65 and over-exhibit significantly lower MAT rates than younger patients.

Third, we find that a 2016 federal rule change that raised the limit on the number of buprenorphine patients per provider (from 100 to 275) may have been critical in enabling some prescribers to reach more patients. A separate federal policy from 2016 that enabled mid-level practitioners (such as physician assistants) to obtain permission to prescribe buprenorphine was also found to be effective in that newly eligible practitioners in Rhode Island did start prescribing the drug, drawing in patients in high-poverty Zip codes in particular. However, the data also suggest that many more patients in the state could be treated with buprenorphine under current policies if existing prescribers took full advantage of their prescribing limits.

The remainder of the paper is organized as follows. Section II provides relevant background information. Section III describes the data source and preliminary sample selection criteria. Section IV describes the methods and results of the analysis of opioid overdose risk. Section V presents the analysis of disparities in MAT uptake. Section VI presents evidence from Rhode Island concerning the effectiveness of policies to promote increased prescribing of buprenorphine for OUD. Section VII concludes with a discussion of policy implications and questions for future research.

\section{Scientific and Policy Background Pertaining to MAT}

\section{A. Opioid Use Disorder and Medication-assisted Treatment}

The Diagnostic and Statistical Manual of Mental Disorders, Fifth Edition (DSM-5) defines opioid use disorder (OUD) as "a problematic pattern of opioid use that leads to serious impairment or distress." (American Psychiatric Association 2013). The condition is also characterized as a chronic brain disease rooted in neurobiology (Volkow et al. 2016). The DSM-5 includes a list of symptoms used to diagnose an individual with either mild, moderate, or severe OUD according to the number and type of symptoms they display. A diagnosis of opioid 
dependence - a category that captures those with either moderate or severe OUD — is often required by insurance companies before they will cover for medication-assisted treatment (MAT) and other treatments for OUD (American Society of Addiction Medicine 2017). According to research, many cases of OUD go undiagnosed (Barocas et. al 2018).

MAT refers to a class of treatments for OUD involving the FDA-approved medications methadone, buprenorphine, and naltrexone, used either alone or in combination. The term "medication-assisted treatment" refers to the fact that such medications are typically applied in conjunction with individual and/or group counselling and other recovery support services. ${ }^{8}$ Methadone and buprenorphine suppress cravings for opioids by occupying the same receptors in the brain that opioid drugs would otherwise occupy, but without producing a euphoric high when used as directed. Naltrexone suppresses cravings for opioid drugs by blocking, rather than occupying, the brain's opioid receptors and cannot produce euphoria. ${ }^{9}$ Daily treatment with methadone or buprenorphine for an extended time period (following an initial detoxification period from other opioids) is referred to as opioid agonist treatment (OAT), and it has been found to be most effective for achieving long-term abstinence from opioids of abuse (World Health Organization 2009; Krantz and Mehler 2004). ${ }^{10}$ The decision of whether to prescribe methadone, buprenorphine, or naltrexone will be specific to each patient and will take into account, for example, the risks of side effects and interactions with other medications (McCanceKatz, Sullivan, and Nallani 2010). Due to data limitations we do not include naltrexone in the present analysis. ${ }^{11}$

\footnotetext{
${ }^{8}$ See "MAT Medications, Counseling, and Related Conditions," Substance Abuse and Mental Health Services Administration.

${ }^{9}$ The injectable form of naltrexone (brand name Vivitrol) has been found to reduce illicit drug use, while the pill form of naltrexone has not been found to consistently improve patient outcomes. See, for example, Lee et al. (2018) and National Institute on Drug Abuse (2020).

${ }^{10}$ The use of medications for opioid use disorder is also abbreviated as MOUD, especially in cases when complementary behavioral treatments are not applied or when the status of complementary treatments is unknown. MOUD includes methadone, buprenorphine, and naltrexone, whereas OAT includes only methadone and buprenorphine.

${ }^{11}$ According to official Rhode Island data, only about 2 percent as many patients in the state receive naltrexone for OUD as receive either methadone or buprenorphine. Therefore, our estimates of the extent of MAT in Rhode Island are not grossly distorted as a result of omitting naltrexone patients.
} 


\section{B. Barriers to Treatment}

Both methadone and buprenorphine are long-acting opioid agonists. Although these drugs differ from short-acting opioids such as heroin and oxycodone that produce a sudden euphoric high, they are nonetheless capable of being abused for non-therapeutic purposes. Methadone and buprenorphine therefore have street value and are subject to diversion. ${ }^{12}$ Accordingly, strict federal and state regulations govern their use in medical applications (Institute of Medicine 1995). These regulations have the effect of limiting patients' ability to access these medications - for example, by requiring that patients travel daily to a specialized opioid treatment program (OTP) to receive methadone and by requiring that health-care providers undergo extra training and obtain a waiver from the Drug Enforcement Administration (DEA) in order to prescribe buprenorphine. ${ }^{13}$ Also due to regulations, OTPs that dispense methadone and office-based practices that prescribe buprenorphine face more expensive staffing requirements compared with those that do not offer these medications (Knudsen et al. 2011). Some states impose caps on the number of facilities that can be certified to dispense methadone, and even without such caps, few communities are willing to permit methadone clinics. For all of these reasons, many patients, especially those in rural areas, face geographic barriers to accessing facilities that dispense methadone and/or providers that are qualified to prescribe buprenorphine (Pullen and Oser 2014; Johnson, Mund, and Joudrey 2018). Despite relaxation over time of policies governing prescribing of buprenorphine, it is estimated that fewer than 1 in 10 primary care providers nationally have waivers (McBain et al. 2020).

Also stemming from the fact that methadone and buprenorphine are opioid agonists, MAT suffers from the stigma that it substitutes one addiction for another. To cite just one powerful example of this stigma, methadone and buprenorphine use is discouraged within the 12-step recovery community, and patients taking these medications are not considered "truly sober."14

\footnotetext{
${ }^{12}$ Fatal overdoses involving methadone do occur, although the rate of such overdoses declined significantly between 2007 and 2014 (Faul et al. 2017).

${ }^{13}$ Some patients can get take-home doses of methadone, but only on a limited basis (Department of Health and Human Services 2001; Walley et al. 2012). Depending on their professional credentials, not all practitioners need to complete special training in order to prescribe buprenorphine, but all would-be prescribers must apply for and obtain a waiver from the DEA. For more details on the regulations governing buprenorphine prescribing, see Burke and Sullivan (2020).

${ }^{14}$ See Katrine J. Andersen and Cecilie M. Kallestrup, "Rejected by A.A.: How the 12 Step Program and Its Decades-old Philosophy Are Exacerbating the Opioid Crisis," The New Republic, June 27, 2018.
} 
To counter this stigma, health authorities, including the National Institute on Drug Abuse (NIDA), stress that although patients on opioid agonist therapy exhibit physical dependence on these drugs - they will experience withdrawal symptoms if they stop taking them - they are not addicted to them in a way that causes self-destructive or criminal behavior and can therefore regain normal social functioning. Furthermore, to combat the broader social stigma that prevents substance abuse sufferers from seeking treatment, the NIDA and other authorities argue that OUD is a chronic brain disease, not a moral failure, and that the treatment of OUD should be viewed through the same lens used to view the treatment of other chronic conditions, such as diabetes and hypertension. ${ }^{15}$

Cost is another inhibiting factor, as not all health insurance plans cover MAT. Before 2020 Medicare plans did not cover outpatient methadone treatments, ${ }^{16}$ and Medicaid plans do not cover methadone in the 12 states that have not adopted the Medicaid expansion. One source estimated that, without insurance, the average cost of methadone treatments and complementary services came to \$126 per week as of 2018 (National Institute on Drug Abuse 2020). The retail price of Suboxone, a popular formulation of buprenorphine, runs from $\$ 6$ to $\$ 24$ per day. ${ }^{17}$ Even when insurance covers MAT, some providers choose not to accept patients with some types of insurance (often Medicaid) due to inadequate reimbursement rates, and some refuse to accept any form of health insurance (Knudsen and Studts 2019; Flavin et al. 2020). Many states, including Rhode Island, offer targeted subsidies for methadone to uninsured residents, although such subsidies are not on par with comprehensive health insurance benefits for substance abuse treatment. $^{18}$

\footnotetext{
${ }^{15}$ See National Institute on Drug Abuse (2018).

${ }^{16}$ See Centers for Medicare and Medicaid Services, "Trump Administration Takes Steps to Expand Access to Treatment for Opioid Use Disorder," November 1, 2019.

${ }^{17}$ See, for example, OpioidTreatment.net, "Does Insurance Cover the Cost of Suboxone (Buprenorphine) Treatment?" July 22, 2018. Suboxone also includes the opioid antagonist Naloxone to prevent abuse. Although methadone alone is inexpensive, the out-of-pocket cost of comprehensive treatment in an OTP, including methadone administration and related treatment services, may run as high as $\$ 125$ per week. See, for example, Alison Knopf, "How Treatment in an OTP Is Paid for: It Costs a Lot More than the Price of the Medication," Addiction Treatment Forum, April 18, 2016.

${ }^{18}$ The federal government's Substance Abuse Prevention and Treatment Block Grant (SABG) program makes funds available to states for the prevention and treatment of opioid use disorder and other substance abuse disorders. In Rhode Island, some portion of these funds are used to help uninsured patients pay for methadone and related services. For more information on the SABG program, see https://www.samhsa.gov/grants/block-grants/sabg and https://bhddh.ri.gov/sections/block_grant.php.
} 


\section{Policies Promoting Increased Access to MAT}

In the past decade, a variety of policies at the federal level and in Rhode Island have been enacted with the goal of helping more patients gain access to health insurance in general and to expand access to medication-assisted treatment in particular. These policies include the Patient Protection and Affordable Care Act (ACA) and its associated Medicaid expansion, and a series of policies related to the prescribing of buprenorphine for OUD. As a result of the ACA, the number of Rhode Islanders without health insurance declined by more than half between 2013 and 2016. ${ }^{19}$ Most of that decline was accounted for by the Medicaid expansion, which brought in nearly 90,000 new enrollees. ${ }^{20}$ These enrollees gained access to generous benefits for mental health and substance abuse treatment services, including full coverage for methadone treatment in specialized opioid treatment programs (OTPs) and full coverage of buprenorphine prescriptions. Mental health parity requirements under the ACA increased the coverage of substance abuse treatment among commercial insurance plans as well. ${ }^{21}$

In 2002, the FDA approved buprenorphine for the treatment of OUD, and the Drug Addiction Treatment Act of 2000 (DATA 2000, which took effect in 2002) allowed psychiatrists and primary care physicians (MDs) to become qualified to prescribe the drug to a limited number of patients. Subsequent federal policies in 2016 (discussed in detail in Section VI) and 2018 expanded the set of providers that can seek permission to prescribe buprenorphine and increased the limits on the number of patients allowed per provider. By enabling office-based practitioners to prescribe an OUD medication for take-home use, these policies gave patients a much more convenient and discreet option for accessing MAT compared with having to make daily visits to an OTP for methadone.

\footnotetext{
${ }^{19}$ See US Census Bureau, "Health Insurance in the United States: 2018 Tables," September 10, 2019.

${ }^{20}$ Under the expansion, the state extended Medicaid managed care coverage to childless adults with incomes at or below 138 percent of the poverty level, pregnant women with household incomes as high as 253 percent of the poverty level, and children in households with incomes as high as 261 percent of the poverty level. Previously, Medicaid was available only to parents with incomes at or below 138 percent of the poverty level, disabled adults, low-income seniors (as a supplement to Medicare), and others needing long-term care and special supports. Also, prior to the expansion, children in families with incomes as high as 261 percent of the poverty level and pregnant women with similar income levels received benefits under the Children's Health Insurance Program, or CHIP. See Louise Norris, "Rhode Island and the ACA's Medicaid Expansion," Healthinsurance.org, October 10, 2020.

${ }^{21}$ For details, see Centers for Medicare \& Medical Services, The Mental Health Parity and Addiction Equity Act (MHPAEA), https://www.cms.gov/CCIIO/Programs-and-Initiatives/Other-Insurance-Protections/mhpaea factsheet.
} 
Under the leadership of Governor Gina Raimondo, Rhode Island enacted a variety of measures to expand access to MAT. ${ }^{22}$ The state in 2016 became the first in the country to offer MAT to prison inmates, a measure that has been credited with reducing overdose deaths (Green et al. 2018). Between 2016 and 2020, Rhode Island established 14 centers of excellence offering a high standard of care for OUD, including MAT and other evidence-based treatments. In 2017 the state formed a partnership with Brown University's Warren Alpert Medical School to incorporate training in addiction treatment into the curriculum and allow graduates who remain in Rhode Island to earn automatic waivers to prescribe buprenorphine; the program became the model for a similar provision included in federal legislation in $2018 .{ }^{23}$ In 2019 one of the largest MAT providers in the state began allowing patients to initiate buprenorphine treatment via a video call, ${ }^{24}$ and subsequent suspension of federal restrictions in response to the COVID-19 pandemic means that patients in Rhode Island can initiate buprenorphine through an audio-only call. ${ }^{25}$

In addition to its proactive policy stance, the state receives significant federal funds for fighting the opioid crisis and allocates a large share of these funds to treatment and recovery programs. ${ }^{26}$ As of 2018, Rhode Island was among the top seven states in the country for methadone clinics per capita, ${ }^{27}$ and as of 2012 the state had more than twice the national average of buprenorphine prescribers per capita (Jones et al. 2015). According to the National Survey of Substance Abuse Treatment Services (N-SSATS), in 2019 roughly 35 percent of all US patients treated for any substance use disorder received MAT, whereas in Rhode Island the rate was more than 59

\footnotetext{
${ }^{22}$ See also Charles Townley, "Rhode Island Becomes Latest State to Pass Opioid Legislation in 2016," National Academy for State Health Policy (blog), July 11, 2016.

${ }^{23}$ See Gary Enos, "Partnership in R.I. Will Accelerate Buprenorphine Training," Addiction Professional, July 13, 2017. The federal legislation that adopted a version of this program was the Substance Use Disorder Prevention that Promotes Opioid Recovery and Treatment for Patients and Communities (SUPPORT) Act of 2018 (Shapiro et al. 2019).

${ }^{24}$ See G. Wayne Miller, "Program Offers Teleconferencing to Treat Substance-abuse Disorders," Providence Journal, June 24, 2019; and "CODAC Behavioral Healthcare and Thundermist Health Center Deploy Technology to Expand Access to Treatment Amid Opioid Crisis," CODAC Behavioral Healthcare, June 17, 2019.

${ }^{25}$ For full directive, see "Electronic Prescriptions for Controlled Substances," US Department of Justice, Drug Enforcement Administration, Diversion Control Division.

${ }^{26}$ See Bipartisan Policy Center, "Tracking Federal Funding to Combat the Opioid Crisis," March 2019, https://bipartisanpolicy.org/wp-content/uploads/2019/03/Tracking-Federal-Funding-to-Combat-the-OpioidCrisis.pdf.

${ }^{27}$ See Christine Vestal, "Long Stigmatized, Methadone Clinics Multiply in Some States," Stateline, October 31, 2018.
} 
percent. Burke (2019) finds that between 2000 and 2017 the share of treatment episodes for OUD that included MAT was consistently higher in Rhode Island than in the United States and also higher than in the other five New England states combined.

\section{Data, Methods, and Sample Selection}

HealthFacts RI is the official name of the state of Rhode Island's all-payer claims database (APCD), a collection of health insurance claims and enrollment data that in any given month intends to capture most Rhode Island residents who hold medical and/or pharmacy insurance. The sample we analyze covers the period April 2011 through May 2019. ${ }^{28}$ Exceptions include people enrolled in plans with fewer than 3,000 total enrollees and, beginning in late 2015, those enrolled in self-insured plans. ${ }^{29}$ The data were obtained under an agreement with the Rhode Island Department of Health (RIDOH), and the study was approved by RIDOH's institutional review board (IRB) and is not considered human subjects research.

For each enrollee observed in a given month the database provides the individual's age, gender, and residential Zip code $;{ }^{30}$ information about their medical and/or pharmacy insurance plans; and extensive information from insurance claims pertaining to the health-care services and prescription medications they received in the month. ${ }^{31}$ The claims data also include information about the providers of the given services and medications, including professional credentials and practice-location Zip codes. ${ }^{32}$ A given individual (whether a patient or a health-care provider) can be followed over time in the data using a consistent numerical code, but the patient data have been stripped of any identifying information in order to protect confidentiality. Data on race and ethnicity are not available. Treatments and medications paid for out of pocket, even by

\footnotetext{
${ }^{28}$ Later data exist but are not currently available to the researchers.

${ }^{29}$ In late 2015 the Supreme Court ruled that self-insured plans could not be mandated to provide claims data to state APCD systems. A self-insured or self-funded plan is a type of coverage offered by some employers where the employer covers the cost of claims as they accrue rather than paying an insurance premium to an insurance carrier. ${ }^{30}$ Throughout the analysis, all Zip codes are converted into their respective Zip code tabulation areas (ZCTAs). ZCTAs are creations of the US Census Bureau that are less prone to variation over time for a fixed physical address; nonetheless in most cases a location's ZCTA is identical to its Zip code. Throughout the text the term "Zip code" can be taken to mean ZCTA. For more information about ZCTAs, see https://www.census.gov/programssurveys/geography/guidance/geo-areas/zctas.html.

${ }^{31}$ To cite just a few data items, we observe detailed codes and descriptions of all procedures, treatments, and diagnoses; standardized drug codes and the quantity supplied for each prescription; payment information such as the enrollee's copay and the full amount charged for each service or medication; and information about the provider that rendered the services.

${ }^{32} \mathrm{We}$ use the provider Zip code only to determine whether a provider is in-state or out-of-state.
} 
individuals carrying insurance, are not included, nor is any information related to dental insurance or dental care.

In the claims data, the different levels of opioid use disorder are coded as either opioid abuse (indicating cases of mild OUD) or opioid dependence (for moderate or severe OUD). A third diagnostic category of "opioid use" (added to the claims coding system in 2015) describes individuals using prescription opioids under medical supervision but not having a disorder, although such individuals may be at risk of developing a disorder. In our broadest definition of OUD we include individuals diagnosed with either opioid dependence, opioid abuse, or opioid use (as in Section IV), although in analyzing uptake of MAT we restrict the sample to those diagnosed with opioid dependence. ${ }^{33}$ In identifying treatment with methadone or buprenorphine, we exclude formulations of either drug used to treat pain. The selection of overdose diagnoses follows the consensus recommendations of the Council of State and Territorial Epidemiologists (CSTE) ${ }^{34}$ See the appendix for the list of diagnostic and procedure codes used in the analysis; the codes are based on the International Classification of Diseases, $9^{\text {th }}$ Revision, Clinical Modification (ICD-9-CM) and the ICD-10-CM (10 ${ }^{\text {th }}$ revision) systems.

We attach information from the American Community Survey (ACS) to construct an indicator of residing in a high-poverty Zip code, which is defined as one where 20 percent or more of the households had income at or below the federal poverty level in the given year-all other Zip codes are deemed "lower-poverty." An individual's Zip code poverty status can change across months or years if they move across Zip codes or if the same Zip code's poverty status changes across years. We also use the ACS to indicate living in a rural Zip code, which is one where fewer than 50 percent of residents live in a town or city with a population of at least 50,000.

Figure 2 illustrates patterns in the coverage of the data relative to the overall Rhode Island population and the insured population from 2012 through 2018 (as fiscal years). The total population of Rhode Island stayed relatively constant in those years, while the share of the population covered by health insurance increased starting in 2014 owing to the Affordable Care

\footnotetext{
${ }^{33}$ Although the opioid use diagnosis does not require disordered use, it is included in our broad definition of OUD because it captures those who may be at risk for more severe disease, and because practitioners may vary in the criteria used to assign different diagnoses. See Barocas et al. (2018).

${ }^{34}$ See Council of State and Territorial Epidemiologists, Drug Overdose Indicator, accessed February 11, 2021, https://resources.cste.org/ICD-10-CM/Drug\%20Overdose\%20Indicator/Drug\%20Overdose\%20Indicator.pdf.
} 
Act and the associated Medicaid expansion. In fiscal 2012, HealthFacts RI enrollees represented 76 percent of all insured Rhode Island residents; by fiscal 2015 that figure had increased to 88 percent, but by fiscal 2018 it had fallen to 77 percent, due to the exit from the sample of enrollees in self-insured plans in fiscal 2016. The share of HealthFacts RI enrollees with Medicaid coverage also increased significantly over the time period, mostly due to the large increase in the number of Medicaid enrollees starting in January 2014. ${ }^{35}$

For the later portions of our sample, external sources can be used to assess the validity of the HealthFacts RI data regarding the number of buprenorphine patients per month, the number of methadone patients per month, and the number of opioid overdoses per month. From January 2016 through December 2018, the HealthFacts RI data capture on average about 81 percent of the total number of buprenorphine patients recorded by Rhode Island's prescription drug monitoring program (PDMP)—-see Figure 3. The PDMP should offer a near census of buprenorphine patients receiving the drug as a prescription. ${ }^{36}$ The coverage rate is lower on average in 2018 than in either 2016 or 2017, because as of January 2018 the HealthFacts RI data lack the prescription drug claims for patients with Medicare fee-for-service insurance. ${ }^{37}$

Our sample's coverage of methadone patients is less complete, as seen in Figure 4. For January 2016 the data capture 59 percent of Rhode Island methadone patients, and that figure declines to roughly 50 percent by June 2016 and hovers close to the latter rate for the remainder of the period. The lower coverage rate for methadone compared with buprenorphine most likely indicates that there are more out-of-pocket payments for methadone than for buprenorphine, consistent with uninsured patients being able to access state block grant funds to pay for

\footnotetext{
${ }^{35}$ In this figure and in most subsequent analyses, the set of Medicaid enrollees excludes those who carry both Medicare and Medicaid insurance simultaneously, a group referred to as dual eligibles. The only exceptions occur in appendix Table A4 and Figure A1.

36 The PDMP observes buprenorphine prescriptions regardless of the form of payment, including out-of-pocket payments. Both the PDMP numbers and the HealthFacts RI numbers exclude individuals receiving buprenorphine in person at an opioid treatment program or in a state correctional facility (American Society of Addiction Medicine 2018). However, most buprenorphine recipients obtain the drug through a prescription. See Cathie E. Alderks, "Trends in the Use of Methadone, Buprenorphine, and Extended Release Naltrexone at Substance Abuse Treatment Facilities: 2003-2015 (Update)," the CBHSQ Report, August 22, 2017.

${ }^{37}$ Starting in January 2019, the HealthFacts RI data also lack medical claims for Medicare fee-for-service patients. The missing data are to be furnished to us at a later date.
} 
methadone treatment. ${ }^{38}$ However, another contributing factor is the apparent redactions —or "scrubbing" - of methadone treatments and OUD diagnoses in our data, especially among claims associated with the insurer United Healthcare. These redactions appear to account for most of the decline in our sample's coverage of methadone treatments in the first half of $2016 .{ }^{39}$ To ensure that these irregularities do not drive our results, we either exclude all observations associated with United Healthcare (as in Section V), or we present results that include such observations and check that those results are robust to their exclusion, as in Section IV.

From February 2016 through December 2018 the monthly counts of opioid overdoses treated in the emergency department (ED) in our data capture 52 percent to 83 percent of all ED-treated overdoses in Rhode Island, depending on the month, or roughly 68 percent on average for that nearly three-year period (Figure 5). The most likely explanation for this undercount is that a disproportionate share of overdose patients lack health insurance and therefore don't appear in our sample. ${ }^{40}$ Some nontrivial number of opioid overdose patients are never transferred to a hospital but instead receive treatment in an ambulance or on the street or receive no treatment whatsoever (Larochelle et al. 2018). Our data do not capture such cases, but neither do the official numbers of ED-treated overdoses.

To build the samples for the following analyses, we first impose restrictions that remove data irregularities and capture an extended picture of each individual's medical history. This process excludes observations in which the medical insurance type is unknown and then requires that the individual (a) is observed in most months from January 2013 through December 2015 (missing as many as three) in addition to any other months, (b) was at least 19 years of age and residing in Rhode Island as of January 2013, (c) did not move out of state after January 2013, and (d) did not switch between Medicaid and non-Medicaid insurance plans (in either direction) before

\footnotetext{
${ }^{38}$ The block grant funds were obtained by Rhode Island through the federal government's Substance Abuse Prevention and Treatment Block Grant (SABG) program. For more information on the SABG program see https://www.samhsa.gov/grants/block-grants/sabg and https://bhddh.ri.gov/sections/block grant.php.

${ }^{39}$ There might be additional redactions in our data set that we are unable to detect, for example if the rate of redactions were consistent over time rather than arising abruptly in selected time periods. Researchers have found evidence of scrubbing of substance use disorder-related claims in other data sets (Rough et al. 2016).

${ }^{40}$ Another potential source of undercounting in our numbers relates to how we count multiple overdoses for the same patient that occur within a short time span. In our method, to count as a new overdose for the same patient, the event must have occurred at least two days after the previous event. We do not know how such cases are counted in the official numbers. We also omit overdoses involving patients who were younger than 18 years old at the time.
} 
January 2017. ${ }^{41}$ A small number of individuals are excluded—less than 1 percent of either the unrestricted sample or the incumbent sample - because they exhibit inconsistent age changes or gender changes over time. The resulting incumbent panel data set includes more than 36 million person-by-month observations pertaining to over 418,000 unique individuals. The term incumbent refers to the fact that all selected individuals had health insurance in Rhode Island prior to the Affordable Care Act and Medicaid expansion of 2014. As described below, the analysis samples used in the overdose analysis and the MAT analysis, respectively, represent subsets of the incumbent panel. The analysis of buprenorphine prescribing draws on a less restricted sample that is described in Section VI.

\section{Repeated Overdose Analysis}

Previous research based on clinical trials indicates that treatment with either methadone or buprenorphine promotes abstinence from illicit opioids and reduces the risks of opioid misuse and all-cause mortality (Connery 2015). Accordingly, it is expected that medication-assisted treatment (MAT) should also reduce the risks of opioid overdose, whether fatal or nonfatal (National Academies of Sciences, Engineering, and Medicine 2019). However, evidence on the association between MAT and opioid overdose risk comes from a relatively small set of observational studies, and while some find that MAT reduces overdose risk (Larochelle et al. 2018; Pierce et al. 2015, Ma et al. 2018), others yield mixed results (Sordo et al. 2017; Kelty and Hulse 2017). Therefore, more research is needed to assess the effectiveness of MAT in reducing overdose risk.

Our approach analyzes repeated overdose risk among patients with at least one nonfatal overdose, in order to eliminate unobserved differences in illness severity that would also predict a higher chance of receiving MAT. ${ }^{42}$ However, this restriction means that the results may not generalize to the question of whether MAT can reduce the risk of having at least one overdose as

\footnotetext{
${ }^{41}$ Some enrollees have non-Rhode Island Zip codes for all or part of the sample period, despite being covered by an insurer that reports to the Rhode Island all-payer claims database. It cannot be determined why these individuals are covered by reporting insurers, but valid reasons could include working in Rhode Island or having insurance through parents living in the state.

${ }^{42}$ Our data indicate that individuals who have had at least one overdose are also much more likely to have ever received MAT compared with those who have never had an overdose. This association could arise because (a) having an overdose reveals the severity of an individual's opioid disorder and prompts health-care providers to recommend MAT, and/or (b) those at higher risk of having an overdose may exhibit signs of a more severe disorder — and therefore be prescribed MAT at higher rates — even before having their first overdose.
} 
opposed to none. We label an overdose as nonfatal if the patient is observed in the sample at least once subsequent to the month of the initial overdose event; otherwise the outcome of the overdose is considered unknown. ${ }^{43}$ The nonfatal opioid overdose sample consists of members of the incumbent panel (described above) who had at least one nonfatal opioid overdose and who were observed (at a minimum) in each of the three months immediately following the initial overdose. The sample contains 70,886 patient-by-month observations ${ }^{44}$ pertaining to 2,079 unique individuals, 539 of whom (about 26 percent) are observed to have had two or more opioid overdoses. ${ }^{45}$ All sample members were at least 18 years of age as of their first overdose.

We estimate the association between recent treatment with either methadone or buprenorphine and the risk of repeated overdose using Cox proportional hazard models. The model assumes that a given factor - such as having ever received medication-assisted treatment (MAT) — multiplies a baseline hazard function by a constant value for any amount of elapsed time. The baseline hazard is estimated nonparametrically setting all explanatory factors at their designated baseline values (given below). Assuming all explanatory factors are fixed over time for an individual, the instantaneous hazard function, $h_{i}($.$) , at analysis time t$ and for a fixed vector of individual characteristics, $\boldsymbol{X}_{\boldsymbol{i}}$, can be written as follows:

$$
h_{i}\left(t \mid \boldsymbol{X}_{\boldsymbol{i}}\right)=h_{0}(t) \exp \left\{\boldsymbol{X}_{\boldsymbol{i}}^{\prime} \boldsymbol{\beta}\right\}
$$

In the above equation $h_{0}(t)$ stands for the baseline hazard function, $\exp$ stands for the natural exponential function, and $\boldsymbol{\beta}$ is the parameter vector to be estimated. The model is readily extended to allow for explanatory factors that vary over time for an individual (Cleves, Gould, and Marchenko 2016). ${ }^{46}$

\footnotetext{
${ }^{43}$ We include overdose events treated either in emergency departments or in hospital settings. The appendix provides the complete set of diagnostic codes used to identify opioid overdoses in the claims data.

${ }^{44}$ The analysis sample size refers to just the observations dated after the first overdose for each person. In defining the medical diagnosis variables and other factors, we also draw on observations from before the first overdose; including all observations before and after the first overdose, there are more than 191,000 person-month observations.

${ }^{45}$ All overdose diagnoses in the same calendar month are treated as the same overdose event. Across different calendar months the overdose diagnoses must be separated by at least two days (with no intervening diagnoses) to be considered separate events. Cases of second overdoses in our sample may or may not have been fatal - it cannot be determined from the data.

${ }^{46}$ As our overdose dates are discretized to the month, we have multiple repeated overdoses occurring at the same time, called "tied failures." As recommended when using discrete failure times, we use the exact partial likelihood
} 
The explanatory factor of interest is the number of months out of the three months immediately preceding the overdose in which the individual either received methadone maintenance treatment or filled a prescription for buprenorphine. For those who ever receive MAT, this variable can change over time, ranging from a minimum of zero to a maximum of three; for those who never receive MAT, the value is always zero. ${ }^{47}$ The model also includes several (time-invariant) indicators of the patient's diagnostic and treatment history, such as ever receiving MAT; ever being diagnosed with alcohol use disorder, opioid use disorder, ${ }^{48}$ another substance use disorder, hepatitis C, any mental illness, depression, or anxiety; ever filling a high-dose opioid drug prescription; ${ }^{49}$ ever filling both an opioid prescription and a benzodiazepine prescription in the same month; and ever receiving behavioral therapy. ${ }^{50}$ Other time-invariant patient factors include having entered the HealthFacts RI sample in 2012 (as opposed to 2011), and the patient's age range as of their first opioid overdose. Gender is accounted for via stratification-separate baseline hazard functions are estimated for women and men - as diagnostic tests indicate that gender does not satisfy the proportional hazard assumption.

The model also includes the time-varying indicators of filling a high-dose opioid prescription in the current month, filling both an opioid and a benzodiazepine prescription in the current month (regardless of dosages), and the number of months out of the preceding three months in which the individual received behavioral therapy. We include an indicator of having Medicaid insurance (as opposed to a non-Medicaid plan) in a given month, although Medicaid status does not change over time for 95 percent of sample members. ${ }^{51}$ Finally, we include fiscal year

\footnotetext{
method of tiebreaking. However, this method precludes obtaining robust standard errors. As a robustness check, we run alternative versions of the models using the Breslow tiebreaking method, which is compatible with obtaining robust standard errors. Results are not meaningfully different under those alternative specifications.

${ }^{47}$ Given the lack of variation in the recent MAT variable among never-MAT recipients, as a robustness test we estimate the model excluding those who never received MAT, and results are very similar.

${ }^{48}$ The opioid use disorder group includes anyone who ever had a diagnosis of either opioid use, opioid abuse, or opioid dependence. The overwhelming majority of this group - 92 percent—received a diagnosis of opioid dependence at least once. Including separate indicators for opioid dependence and opioid use or abuse does not significantly alter the regression results.

${ }^{49} \mathrm{~A}$ high-dose opioid prescription is defined as one involving a morphine milligram equivalent dose of 90 or greater, not including opioid agonist medications used to treat OUD.

${ }^{50}$ The complete set of ICD-9 and ICD-10 codes used to identify the conditions and treatments listed in this paragraph are given in the appendix. The list of drug codes used to identify opioids and benzodiazepines can be obtained upon request from the authors.

${ }^{51}$ This statement refers to changes in Medicaid status during the period of time following the first overdose, as Medicaid status in the time period before the first overdose does not enter the hazard model estimation.
} 
indicators to control for secular trends in the risks of repeated overdose not captured by other variables; their coefficients are in most cases insignificant and are suppressed from all tables.

For all binary indicators in the model, the baseline value is zero. Baseline values are also zero for number of last three months with MAT and number of last three months with behavioral therapy. The baseline insurance category is non-Medicaid, the baseline age range as of the first overdose is ages 18 through 30 , the baseline fiscal year is 2012 , and the baseline sample entry year is 2011.

Table 1 describes the sample means of the variables used in the model (and some additional indicators) for the entire sample combined and then separating those with a single opioid overdose from those with two or more opioid overdoses. These measures draw on all of an individual's observations, including those dated prior to the first overdose. Close to half of all sample members received MAT (either methadone or buprenorphine) at least once, but the share of MAT recipients is significantly greater among patients with at least two overdoses than among those with a single overdose. This association suggests that eventual overdose repeaters are identified as having more severe disorders even in advance of their second overdose and are therefore prescribed MAT at a higher rate. This possibility underlines the importance of controlling for "ever-MAT" status when estimating the relationship between recent MAT and repeated overdose risk. Among all sample members who received MAT, more than two-thirds initiated MAT prior to their first opioid overdose.

Also as seen in Table 1, overdose sample members are highly likely -85 percent or more, depending on the condition - to have been diagnosed with anxiety, depression, another substance use disorder, or another mental illness besides opioid use disorder (OUD), and nearly one-third have been diagnosed with hepatitis $\mathrm{C}$. The sample is not restricted to those who have been diagnosed with OUD, but close to 70 percent displayed a diagnosis of opioid dependence and about 6 percent had a less severe diagnosis of either opioid use or opioid abuse. More than half of the sample members have on at least one occasion filled prescriptions in the same month for an opioid and an anti-anxiety drug (benzodiazepine), which are known to be highly dangerous when combined (Gudin et al. 2013). More than half of the sample members are male, and more than one-third had their first observed overdose at age 53 or older. About 44 percent had 
Medicaid insurance as of January 2015 - compared with roughly one-fourth of all HealthFacts RI enrollees as of the same date..$^{52}$

Table 2 shows the estimated hazard ratios from four different specifications of the stratified proportional hazard model described above. The instantaneous risk of having a second overdose (any number of months after the first overdose) decreases with the number of MAT treatments in the preceding three months. However, when we do not control for whether the patient ever received MAT (column 1), this association is weak and statistically insignificant, most likely due to confounding with the omitted risk factors that drive selection into receiving MAT. Based on the model in column 3, which includes the full set of controls, a patient who received MAT in exactly one of the preceding three months is expected to face a 15 percent lower risk of repeated overdose compared with a patient with no MAT in the preceding three months (but who received MAT at least once outside of that period); and a patient with MAT in all of the preceding three months would have a 38 percent lower risk relative to a patient in that same comparison group. Characteristics associated with significantly elevated risk of repeated overdose include ever receiving MAT, ever having an opioid use disorder diagnosis, ever having an alcohol use disorder diagnosis, and ever having a hepatitis $\mathrm{C}$ diagnosis. Age at first overdose (measured as a discrete age range) is negatively associated with repeated overdose risk. To cite the starkest difference, those ages 43 to 52 as of their first overdose experience a nearly 40 percent lower (instantaneous) risk of repeated overdose compared with those ages 18 to 30.

Figures 6 shows estimates of the cumulative risks of repeated overdose for women as a function of the months elapsed since the first overdose and assuming various combinations of characteristics, based on the model in column 3 of Table 2 . All the curves in the figure pertain to a female patient who was diagnosed with opioid use disorder and received MAT at least once, but alcohol use disorder status and the number of recent MAT treatments varies. All other variables are held at their baseline values. ${ }^{53}$ Among the types represented, the patient who was

\footnotetext{
${ }^{52}$ Based on how the sample is constructed, those with Medicaid insurance as of January 2015 would have held Medicaid insurance in all or most of their observations, although about 5 percent of sample members switched away from Medicaid sometime after December 2016.

${ }^{53}$ For all binary indicators in the model, the baseline value is zero. Baseline values are also zero for the number of last three months with MAT and the number of last three months with behavioral therapy. The baseline insurance
} 
never diagnosed with alcohol use disorder and who received MAT in all three of the preceding three months faces the lowest cumulative risk of repeated overdose any number of months after the first overdose. In contrast, a patient with alcohol use disorder who has not received any MAT in the preceding three months experiences the highest risks. The model predicts a roughly 16 percent chance that the latter patient will have had a second overdose within 10 months of the first overdose and only an 8 percent chance that the former patient will have done the same. The two intermediate curves — which are nearly coincident — show that the effect of having alcohol use disorder on repeated overdose risk is roughly equivalent to the effect of having no recent MAT treatments. This offsetting effect means that any risk-lowering effects of MAT would be hard to detect if one were to compare patients without taking into account whether they have alcohol use disorder.

The cumulative risks of repeated overdose for men (Figure 7) differ in their overall shape from those estimated for women, in that risks increase less rapidly for men (and are therefore lower) within the first 20 months. However, after 40 months or more the cumulative risks for male and female patients with the same sets of characteristics are quite close. Consistent with how the model was estimated, the multiplicative effects of the different factors on repeated overdose risk are equivalent to those seen among women.

These results suggest that individuals with more consistent recent MAT treatments experience substantial reductions in repeated overdose risk. The repeated overdose risk profile is steepest in the first 12 months following an initial overdose, suggesting that early treatment intervention is important. Indeed, among overdose repeaters in our sample, almost 14 percent had their second overdose less than one month after their first, 29 percent had their second overdose within less than three months, and the median time lapse between the first and second overdoses is just under nine months. ${ }^{54}$

\footnotetext{
category is non-Medicaid, the baseline age range as of the first overdose is ages 18 through 30 , the baseline fiscal year is 2012, and the baseline sample entry year is 2011.

54 These calculations employ the exact dates of the first and second overdoses, rather than just the month of each overdose. Months between overdoses are calculated as days divided by 30.4 and rounded to the nearest whole number.
} 
Unobserved shocks to health status could confound the association between recent MAT and overdose risk. For example, someone who experiences a life setback might be less capable of showing up to the methadone clinic or filling their buprenorphine prescription and, also due to the setback, might be more likely to resume abusing opioids at levels that pose a higher risk of overdose. As shown in column 4 of Table 2, we control for such shocks by conducting a placebo test of whether overdose risk responds to MAT in the upcoming three months and/or to behavioral therapy in the upcoming three months, either of which might also be indicative of current life circumstances or health status but should not have a causal effect on present overdose risk. The coefficient on the number of recent MAT treatments is highly robust - the point estimate would indicate that if anything, the reduction in risk from recent MAT is greater in this model—whereas the association between either of the placebo treatments and overdose risk is null.

As additional robustness checks we estimate similar models (a) using the Breslow tiebreaking method with robust standard errors, (b) requiring a minimum of six months of follow-up time after the first overdose instead of just three months, (c) on an alternative sample that excludes observations from United Healthcare, and (d) on just the set of individuals who ever received MAT. In all cases we observe a statistically significant negative association between the number of recent MAT treatments and the risk of having a second overdose. (See appendix Table A1 for complete results.)

\section{Treatment Disparities}

The analysis above reinforces medical guidelines that endorse the use of methadone and buprenorphine to treat opioid use disorder (OUD). The next step is to identify factors that might increase or decrease the chances that an OUD patient will receive either of these medications, as this information can inform policies seeking to promote greater uptake of medication-assisted treatment (MAT) to improve patient outcomes.

The literature on disparities in MAT is quite scarce in terms of studies using large administrative data sets, and ours is the first study we are aware of that uses an all-payer claims database to address this issue. Based on a national survey of ambulatory care, researchers find that African American patients are less likely to receive buprenorphine than are white patients, when the 
analysis controls for age, sex, and payment method but not for OUD status (Lagisetty, Ross, and Bohnert 2019). A study of young adults (ages 13 to 25) based on a national commercial health insurance database and adjusting for other factors, finds that among those diagnosed with OUD, Black (non-Hispanic) and Hispanic youths have lower MAT rates (including either buprenorphine or naltrexone) than white youth, and females have rates that are lower than those of otherwise similar males. Using Medicaid claims for the state of New York, Neighbors et al. (2019) find that young adults with OUD are less likely to receive MAT than are older adults with OUD. Analyses of provider location data and small-scale provider surveys indicate that residents of rural areas face limited geographic access to MAT. ${ }^{55}$

To identify the factors that are associated with receiving MAT, we estimate multivariate probit regressions in which the outcome is defined as being treated with methadone (or, separately, buprenorphine) in at least three separate calendar months. ${ }^{56}$ The analysis is restricted to the patients in the incumbent panel (described above) who have ever been diagnosed with opioid dependence, the most severe form of disorder under the OUD umbrella. This restriction reduces the potential heterogeneity in illness severity in the sample, and it controls for the fact that many insurance companies require a diagnosis of opioid dependence as a prerequisite for coverage of MAT. ${ }^{57}$ The set of predictive factors for either outcome consists of the following: the patient's sex, the year of the patient's first observation in the database, the patient's age group as of the first observation, having Medicaid insurance as opposed to non-Medicaid insurance,${ }^{58}$ living in a

\footnotetext{
55 See, for example, Andrilla et al. (2018), Grimm (2020), Pullen and Oser (2014).

${ }^{56}$ A methadone month is one in which the patient has one or more claims that include the procedure code for methadone maintenance treatment (H0020). A buprenorphine month is one in which the pharmacy claims data indicate that the patient filled at least one buprenorphine prescription in that month or had an "open prescription" for buprenorphine on at least one day in that month. A prescription is considered open if the days elapsed since it was filled are less than the total intended days' supply of the prescription as indicated in the claim. We exclude denied claims and claims involving buprenorphine formulations used to treat any condition other than OUD. The list of excluded formulations is given in the appendix, and the list of national drug codes for the allowed formulations can be obtained from the authors upon request.

${ }^{57}$ Before imposing the incumbent panel restrictions and selecting those who have at least one observation with a diagnosis of opioid dependence, we exclude observations in which the individual was younger than 18 , in which the Zip code was not in Rhode Island, and in which the medical insurance payer was either United Healthcare or an unknown payer.

${ }^{58}$ Age group and Medicaid insurance status are based on the patient's insurance as of their earliest observation in 2013. The sample excludes individuals who switched between Medicaid and non-Medicaid plans before December 2016, such that Medicaid status as of early 2013 will apply to most if not all of each patient's observations.
} 
high-poverty Zip code ${ }^{59}$ and indicators of having a diagnosis (ever) of alcohol use disorder, substance use disorder (not including alcohol or opioids), depression, anxiety, mental illness (not including OUD), hepatitis C, and opioid overdose, respectively. We analyze treatment with methadone separately from treatment with buprenorphine because we find that these two outcomes differ in terms of how each is associated with the predictive factors.

The probit model for either binary outcome, denoted $Y_{i}$, can be expressed as follows:

$$
P\left(Y_{i}=1 \mid \boldsymbol{X}_{\boldsymbol{i}}\right)=\Phi\left(\boldsymbol{X}_{\boldsymbol{i}}^{\prime} \boldsymbol{\beta}\right)
$$

In the equation above, $\boldsymbol{X}_{\boldsymbol{i}}$ is the vector of explanatory factors (fixed over time for the individual and including the constant 1$), \boldsymbol{\beta}$ is the parameter vector to be estimated, and $\Phi($.$) stands for the$ cumulative normal distribution function. ${ }^{60}$

Column 2 of Table 3 shows the sample means of the outcomes and the predictive factors over the regression sample, along with sample means for the larger set of enrollees in the newly restricted incumbent panel. The nearly 3,000 enrollees in the opioid dependence subsample comprise about 3 percent of the total enrollees in the restricted incumbent panel. Within the opioid dependence group, just under 20 percent received methadone treatments in at least three separate months, about 29 percent were on buprenorphine in at least three separate months, and almost 43 percent received at least one of these medications in three or more months. ${ }^{61}$ Women are underrepresented in the opioid dependence sample relative to their share of the larger sample, as are those who entered the sample at age 65 or older. Members of the opioid dependence sample are more likely to reside in a high-poverty Zip code and are more likely to have Medicaid insurance compared with the broader sample population. They are also much more likely to have any of the comorbid diagnoses of alcohol use disorder, other substance use disorder, hepatitis C,

\footnotetext{
${ }^{59}$ Using the American Community Survey, we define a Zip code (ZCTA) as "high poverty" in a given year if 20 percent or more of the households had income at or below the federal poverty level in the given year. As each patient is observed in multiple periods and may move across Zip codes over time, a given patient is said to reside in a high-poverty Zip code if they lived in such a Zip code in more than half of their observations.

${ }^{60}$ Results of logit models using the same variables are very similar both quantitatively and qualitatively. However, postestimation analysis indicates that the probit models offer a better fit with the data and stronger predictive power. ${ }^{61}$ Although we do not have measures for the United States that are directly comparable to these MAT rates for Rhode Island, the figure of 43 percent (receiving either methadone or buprenorphine) exceeds the estimate from the 2019 N-SSATS that roughly 35 percent of all US patients treated for any substance use disorder received MAT. Furthermore, the 43 percent figure for Rhode Island is not restricted to patients who were otherwise treated for a substance use disorder, and that restriction would likely lead to an even higher share receiving MAT.
} 
depression, anxiety, and other mental illness. The differences in the diagnosis rates between these groups may overstate the true differences in the prevalence of these conditions, however, as patients diagnosed with opioid dependence may simply be more likely to have had those other conditions diagnosed.

The table also shows typical durations of MAT, calculated as the sample median of the withinperson average number of consecutive months on a given medication. ${ }^{62}$ (Each individual may have multiple spells.) The median treatment duration is longer for methadone (26 months) than for buprenorphine (15 months), but both of these figures conform to or exceed common medical recommendations that patients should stay on MAT for a minimum of 12 months and that longer durations (15 months or more) are associated with improved outcomes (Williams et al. 2020). At the same, time these figures obscure the fact that a nontrivial share (3.5 percent) of patients in the opioid dependence sample had an average MAT duration of just one month, and more than 30 percent had an average duration shorter than 12 months.

Table 4 shows the estimated coefficients from the probit models. All standard errors were estimated using the robust option. Factors with significant positive coefficients indicate that patients with that characteristic are more likely to have been treated with methadone (in column 1) or buprenorphine (in column 2), and vice-versa for negative coefficients. Figure 8 shows the average predicted chance of receiving methadone at selected values of characteristics as estimated by the regression model in column 1 of Table $4 .{ }^{63}$ Opioid dependence patients on Medicaid insurance are more than twice as likely to be treated with methadone compared with patients on non-Medicaid plans. Patients living in high-poverty Zip codes have a higher predicted chance of receiving methadone than do patients in Zip codes with lower poverty rates, by about 2 percentage points on average. Women are less likely than men to receive methadone, but by an expected margin of less than 2 percentage points. In addition, patients with comorbid alcohol use disorder face a much lower chance of receiving methadone compared with patients

\footnotetext{
${ }^{62}$ Each individual may have multiple spells on MAT. To be considered distinct, two methadone spells must be separated by at least one month with no claims involving methadone, and two buprenorphine spells must be separated by at least one month in which the patient had no open prescription or filled prescription for the drug. ${ }^{63}$ For example, the average predicted probability of a Medicaid-insured patient receiving methadone is calculated as follows: A predicted probability of the outcome is generated for each patient in the sample assuming the patient has Medicaid insurance (regardless of their actual insurance) and setting all other characteristics at their actual values and applying the regression coefficients. The respective predicted probabilities are then averaged over all patients in the sample.
} 
who don't have that condition, and patients in older age groups (as of their first observation) are less likely to receive methadone compared with younger patients. ${ }^{64}$ Other factors associated with a higher expected chance of receiving methadone (as seen in Table 4 but not depicted in Figure 8) include having had an opioid overdose, a hepatitis $\mathrm{C}$ diagnosis, or a diagnosis of another substance use disorder. All of the contrasts just described are statistically significant at the 5 percent level or better.

The factors that predict buprenorphine treatment are in some cases similar to those observed for methadone but in other cases strikingly different - refer to Table 4 and Figure 9. Again, women are somewhat less likely to receive buprenorphine compared with men, and older age also predicts a lower buprenorphine treatment rate. In contrast with methadone treatment patterns, patients in high-poverty Zip codes are less likely to receive buprenorphine than are patients in lower-poverty Zip codes, having Medicaid insurance does not predict a significantly greater tendency to be treated with buprenorphine, ${ }^{65}$ and alcohol use disorder is not associated with any difference in the chance of receiving the medication.

The sample was constructed so that each individual had a large number of observations over a similar time period, ensuring accurate measurement of the variables of interest and minimizing the potential influence of time trends in treatment patterns. However, the share of patients with Medicaid insurance in our sample is significantly lower than it would be in a sample that included people who entered after January 2014, when the Medicaid expansion took effect in Rhode Island. Using a less restricted sample that includes later entrants produces results that are mostly very similar. Results are also robust in models in which the dependent variables are defined as having at least one month on methadone and one month on buprenorphine, respectively, rather than at least three months on either medication. ${ }^{66}$

The associations just described are not necessarily causal, and multiple interpretations are possible. Concerning the higher rate of methadone receipt among Medicaid patients, previous research suggests a role for the plan itself, which offers full coverage of methadone (and

\footnotetext{
${ }^{64}$ Differences in sample entry age ranges are good indicators of age differences at a given point in time, as most patients were first observed in April 2011 and all sample members had entered by December 2012.

65 There is a small positive coefficient on the Medicaid indicator, but we aren't highly confident that the effect is actually greater than zero.

66 The results of the models described in this paragraph are shown in appendix Table A2.
} 
buprenorphine) and associated behavioral therapy. ${ }^{67}$ However, methadone treatment facilities are disproportionately likely to be located in high-poverty Zip codes, where Medicaid patients are also more likely to reside. ${ }^{68}$ By controlling for residing in a high-poverty Zip code—which does indeed predict a higher rate of methadone treatment-we reduce the chance that proximity to a methadone facility explains the effect of Medicaid on methadone treatment. ${ }^{69}$ The fact that having Medicaid insurance predicts a higher methadone rate but not also a higher buprenorphine rate suggests that Medicaid patients may find it easier to access methadone relative to buprenorphine. Again, geography may play a role, consistent with the fact that living in a highpoverty Zip code is negatively associated with the chance of receiving buprenorphine. However, prior research also finds that buprenorphine providers may be reluctant to accept Medicaid patients (Knudsen and Studts 2019).

Concerning women's lower chances of receiving MAT, we find that the difference derives entirely from women who had no observed pregnancies (even when we restrict to women of childbearing age), whereas women with at least one pregnancy had similar chances of receiving MAT compared with otherwise similar men. Therefore, pregnancy does not appear to be a deterrent to receiving MAT. Also, although women in our sample are more likely to have taken benzodiazepine drugs (which can interact negatively with either methadone or buprenorphine), that fact does not explain the gender gap in MAT, as the effect of being female is robust to our including an indicator of ever having taken benzodiazepines. ${ }^{70}$

Medicare did not cover the cost of outpatient methadone treatment until 2020, a disadvantage that would have restricted methadone access for those age 65 and older (as well as for younger Medicare beneficiaries). Further investigation reveals that methadone rates decline sharply with age even among Medicaid enrollees, suggesting that Medicare insurance alone can’t explain the

\footnotetext{
${ }^{67}$ See, for example, Maclean and Saloner (2017), Clemans-Cope et al. (2017), and Wen et al. (2017).

${ }^{68}$ Using data from the Substance Abuse and Mental Health Services Administration (SAMHSA) indicating the Zip codes (ZCTAs) of the 23 OTPs listed in Rhode Island in 2020, together with our indicators of Zip code (ZCTA) poverty status as of 2018, we find that as of 2020 two-thirds (4 out of 6) of the state's high-poverty ZCTAs had at least one OTP, while fewer than 20 percent (14 out of 71) of the state's lower-poverty ZCTAs housed at least one such facility.

${ }^{69}$ Another possibility is that non-Medicaid insurance plans were more prone to redact or suppress methadone treatments from the claims data they submitted to HealthFacts RI, although there is no way to test that hypothesis.

${ }^{70}$ Results are shown in appendix Table A3.
} 
observed age disparities in methadone treatment. ${ }^{71}$ Furthermore, buprenorphine rates also decline sharply with age (as seen in Figure 9), even though buprenorphine coverage is relatively uniform across the insurance plans in our data. The literature on older age and MAT is quite sparse, but at least two studies note that older OUD patients may experience even greater stigmatization for their condition than younger patients, a factor that may deter their seeking treatment. ${ }^{72}$ Recall also that the analysis above finds that repeated overdose risks are significantly lower for patients who were older as of their first overdose, even after we condition on recent MAT status and numerous comorbidities. If older OUD patients generally face lower risks of overdose and providers are aware of this fact, their lower rates of MAT may be at least partly explained on that basis.

The fact that there are significant health risks from mixing alcohol and methadone may explain why OUD patients with alcohol use disorder (AUD) are less likely to receive methadone. However, it remains puzzling that AUD patients are not less likely to receive buprenorphine, which also may interact adversely with alcohol. It is possible that OUD patients with AUD are being treated with the opioid antagonist drug naltrexone, which does not cause dangerous interactions with alcohol. However, naltrexone use is likely to explain only a small fraction of the methadone gap among AUD patients, as the number of OUD patients in Rhode Island treated with naltrexone is very low according to the official state numbers. ${ }^{73}$

Finally, we did not find evidence of treatment disparities based on living in a rural Zip code, and so we dropped that indicator from the model—see Appendix table A5 for results including that indicator. The proportion of patients in the regression sample residing in such Zip codes (during a majority of their months in sample) was just over 5 percent.

\section{Effectiveness of Policies to Expand Access to Buprenorphine}

Two significant changes to federal policies governing the prescribing of buprenorphine were enacted in mid-2016; both had the goal of helping more opioid use disorder (OUD) patients gain access to buprenorphine. In late July of 2016, the US Congress passed the Comprehensive

\footnotetext{
${ }^{71}$ See appendix Table A4 and Figure A1 for these results.

72 See Conner and Rosen (2008) and Anderson and Levy (2003).

${ }^{73}$ See Prevent Overdose RI, Medication-assisted Treatment Data, accessed February 5, 2021, https://preventoverdoseri.org/medication-assisted-therapy.
} 
Addiction and Recovery Act (CARA), which enables nurse practitioners and physician assistants to obtain waivers to prescribe buprenorphine-previously only MDs and psychiatrists could get such waivers. In early August of 2016, the Substance Abuse and Mental Health Services Administration (SAMHSA) enacted a final rule that allows qualified incumbent prescribers to increase their maximum patient limit to 275 after gaining one year of experience treating 100 patients. See Burke and Sullivan (2020) for a detailed timeline of federal buprenorphine policy changes. The former policy is aimed at increasing the number of buprenorphine providers, and the latter targets the number of patients served per provider-offering two channels for boosting the total number of patients treated with the drug. An increase in the number of waivered providers of buprenorphine has been associated with increased rates of buprenorphine prescribing as well as decreased rates of opioid prescribing (Wen et al. 2018). The analysis that follows seeks to determine whether, during the sample period, these policies delivered the intended effects, and whether either policy proved more important than the other in terms of helping to increase patients' access to buprenorphine in Rhode Island. ${ }^{74}$

We focus on changes in prescribing activity on either side of the policy changes. Allowing for a one-month lag until the changes had any effects, we let the "post-policy" period start in September 2016 and run through August 2017, ${ }^{75}$ a total of 12 months, and we restrict the "prepolicy" period to September 2015 through August 2016, a 12-month period that includes the two months when these laws were enacted. Although the choice of these time intervals is somewhat arbitrary, considering symmetric time periods on either side of the policy changes is desirable for its own sake, and setting the first month of the pre-policy period as September 2015 should also help to minimize the influence of the Affordable Care Act (ACA) and Medicaid expansion on pre-policy trends - the ACA and Medicaid expansion led to a surge in enrollments in 2014 and also helped expand coverage for buprenorphine.

To make comprehensive estimates of prescribing activity, we use a sample with only minimal restrictions as opposed to employing the incumbent panel used in the preceding analyses. In

\footnotetext{
${ }^{74}$ The SUPPORT Act of October 2018 further expanded the pool of eligible providers and allows for higher initial patient limits for qualified providers. However, our data set extends only through May 2019 and is missing some pharmacy claims and insurance enrollment information beginning in January 2018. Therefore, we are not in a position to evaluate the effects of the SUPPORT Act.

75 The pharmacy claims of Medicare fee-for-service patients are missing from the data from January 2018 forward, and as a result the trends in buprenorphine prescribing are also somewhat distorted from that same date on.
} 
addition to the date restrictions noted above, we removed observations in which the patient resided outside of Rhode Island or was not at least 18 years of age, in which the claim for buprenorphine was denied or involved a non-allowable formulation, ${ }^{76}$ and in which the prescribing activity came from an out-of-state providers based on the practice's reported Zip code. $^{77}$

The number of buprenorphine patients per month fluctuated during the 12-month pre-policy period, but on balance it was almost flat; ${ }^{78}$ see Figure 10. In contrast, the monthly patient load increased in September 2016 and trended upward throughout the post-policy period. There were roughly 400 additional buprenorphine patients in an average month in the post-policy period compared with an average month in the pre-policy period. ${ }^{79}$ This significant increase in the number of buprenorphine patients in the post-policy period reflects two underlying developments: The number of providers dispensing the drug increased, as did the average number of patients served per prescriber, as seen in Figure 11. (The dashed vertical line in the figure marks September 2016, the start of the post-policy period.) These facts suggest that both policies achieved some measure of success.

To assess the relative importance of each of these two margins - the stock of prescribers and patients per prescriber - in boosting patient loads, we construct two counterfactual scenarios. In the first scenario we calculate the patient counts (by month) that would have arisen with no change in the average number of patients per prescriber (from its pre-policy six-month average) while allowing the number of prescribers to evolve as observed. The changes in the patient load over time under this scenario reflect only the contribution of changes in the prescriber stock. To

\footnotetext{
${ }^{76}$ An allowable buprenorphine formulation is one that is FDA-approved to treat OUD, and non-allowable formulations are those used to treat pain or another condition. See the appendix for a list of the non-allowable formulations.

${ }_{77}$ We also excluded providers listed as organizations rather than individuals, and we excluded the activity of a single prescriber with exceedingly high patient loads that would have distorted all statistics.

${ }^{78}$ Patients per month is calculated as the number of unique sample members aged 18 and older who filled an allowable buprenorphine prescription in the given month. All results are qualitatively similar if we define a buprenorphine patient as someone with an open buprenorphine prescription during the month. A prescription is considered open if the days elapsed since it was filled are less than the total intended days' supply of the prescription as indicated in the claim.

${ }^{79}$ Over a much longer pre-policy period spanning January 2012 through August 2016, patient loads increased significantly. Nonetheless, the average increase per month in the number of buprenorphine patients (scaled per total enrollees) is modestly greater in the (extended) post-policy period of September 2016 through December 2017 than in the extended pre-policy period of January 2012 through August 2016.
} 
isolate instead the contribution of changes in the average number of patients per prescriber, we hold the prescriber stock fixed at its initial (pre-policy) six-month average and apply the observed monthly values of patients per prescriber.

Figure 12 shows the hypothetical patient load under each of these two scenarios, together with the actual patient load, for each month of the pre-policy and post-policy periods. The analysis reveals that increases in the average number of patients per prescriber account for more than half of the increases in the number of buprenorphine patients between the pre-policy and post-policy periods. Comparing average outcomes in the first six months of the post-policy period (September 2016 through February 2017) with average outcomes in either the first half or the second half of the pre-policy period, about 60 percent of the rise in the total number of patients receiving buprenorphine can be attributed to the uptick in the average number of patients per prescriber, and about 40 percent owes to the increase in the number of prescribers. Regarding average outcomes in the second half of the post-policy period (March 2017 through August 2017) compared with either the first half or the second half of the pre-policy period, again roughly 60 percent of the increase in the patient load can be linked to an increase in patients per prescriber, and about 30 percent reflects an increase in the stock of prescribers - the remaining 10 percent reflects a residual interaction effect between the two margins. ${ }^{80}$

The increase along either margin was modest — the average number of patients per prescriber increased 8.4 percent, from roughly 16.9 in the pre-policy period to 18.3 in the post-policy period, while the average number of prescribers per month increased just 4.7 percent, from 171 to $179 .{ }^{81}$ Recall that before August 2016 a prescriber could obtain permission to serve at most 100 patients, whereas starting in that month, per a SAMHSA rule change, some prescribers (depending on their existing patient limit and their experience or qualifications) became eligible

\footnotetext{
${ }^{80}$ Some commercial insurers became exempt from reporting to HealthFacts RI in late 2015, which results in steep declines in the total number of enrollees in our sample in the first four months of 2016. To control for any distortions in trends caused by this issue, we replicate the counterfactual analysis after dropping from the sample all those who were ever enrolled in commercial medical insurance plans. The revised results are qualitatively robustincreases in the number of patients per prescriber still account for about 60 percent of the increases in patient loads between the post-policy and pre-policy periods.

${ }^{81}$ The increase of 4.7 percent represents the increase in the exact 12-month average number of prescribers between the pre-policy and post-policy periods. Between the first half of the pre-policy period and the second half of the post-policy period, the average monthly prescriber stock rose by a slightly larger margin, from 170 to 181 . Also, as the prescriber stock experiences both entry and exit over time, these changes in the number of active prescribers per month represent net changes that understate the gross number of new entrants over the period.
} 
to seek permission to serve as many as 275 patients. Because the average number of patients served per prescriber in our sample falls well below the threshold of 100 patients, both before and after the rule change of August 2016, it might appear that the rule change was not critical in facilitating the observed increase in that number.

However, examining changes in patient loads per prescriber at a more granular level suggests that some prescribers did take advantage of the increase in the patient limit and that high-volume prescribers were in fact the key drivers of the increase in the average number of patients served per prescriber. Figure 13 shows trends in various percentiles of the patients per prescriber distribution over the pre-policy and post-policy periods. ${ }^{82}$ Only the $95^{\text {th }}$ percentile exhibits a noticeable upward trend, while the lower percentiles - including the median - remain effectively flat or even decline across the periods. Most notably, the $95^{\text {th }}$ percentile crosses the threshold of 100 patients per prescriber beginning in May 2017. That is, the top 5 percent of prescribers in terms of patient volume (a select group of fewer than 10 prescribers) as of the second half of 2017 served more than 100 patients apiece on average.

Even though our data indicate that there was only a modest increase in the number of buprenorphine prescribers in Rhode Island following the passage of CARA - the law that enabled physician assistants and nurse practitioners (so-called mid-level practitioners) to prescribe the drug - the policy appears to have been important in helping to expand buprenorphine prescribing to previously underserved patients or areas. Figure 14 shows the distribution of provider types among patient-provider pairs in the post-policy period, among incumbent patients - those who received buprenorphine before September 2016 - and among new patients - those who first received buprenorphine in the post-policy period. Mid-level practitioners, who effectively had zero market share in the pre-policy period, achieved a significant market share, among incumbent patients as well as new patients. However, they achieved a larger market share among new patients, suggesting that these newly eligible providers may have succeeded in reaching patients who previously had difficulty accessing a buprenorphine provider.

\footnotetext{
${ }^{82}$ The percentile calculations are based on the number of patients (by provider) who filled buprenorphine prescriptions in the given month. Providers with zero patients in a given month are omitted from the percentile calculation in the given month.
} 
Furthermore, as seen in Figure 15, in the post-policy period, the newly eligible providers achieved a greater market share in high-poverty Zip codes than in lower-poverty Zip codes, suggesting that these new providers may have enabled increased access to buprenorphine for patients residing in high-poverty areas. Recall that the analysis in Section VI finds that, on average from 2011 through 2018, such patients were significantly less likely to be treated with buprenorphine compared with patients in lower-poverty Zip codes.

Despite these positive responses of buprenorphine prescribing in Rhode Island to recent federal policies, prescribing activity appears to fall below potential for the typical provider. On average in 2017, the median active prescriber in our sample served an estimated 12 patients in a given month. When we adjust that number upward to account for the fact that our sample captured only about 85 percent of Rhode Island buprenorphine patients on average in 2017 (see Figure 3), the resulting value of 14 still represents less than half of the minimum patient limit of 30 per provider. ${ }^{83}$

Even more concerning, some waivered buprenorphine providers may not use their prescribing privileges at all, or they may not accept health insurance as payment for their services. As seen in Figure 16, in either 2014 or 2015 the number of unique Rhode Island prescribers of buprenorphine observed in our data coincides nearly exactly with the official Rhode Island number reported for the same year, but in 2016 and 2017 our numbers fall short. The official numbers are based on observing waiver status directly, whereas our own counts are based on whether a provider wrote a buprenorphine prescription for at least one patient in our data set during the year and reported a Rhode Island Zip code. ${ }^{84}$ These patterns suggest that in 2014 and

\footnotetext{
${ }^{83}$ The prescribing limits for buprenorphine refer to the number of patients being treated with buprenorphine at any given time by a given individual provider. In this exercise we estimate a prescriber's patient load based on the number of patients who filled a prescription in a given month; prescribers may have had additional patients under their care who were taking buprenorphine as a result of prescriptions that were filled in an earlier month. However, the median patient loads increase by less than half of one patient if we count all patients with an open prescription in a given month. Furthermore, our estimates may be too generous, as the percentile calculations for each month exclude prescribers with zero patients in the month (even if the same prescriber resumed having patients at a later date). Including the zeroes, the median monthly patient load per prescriber was roughly three on average in 2017 , regardless of whether we count patients who filled prescriptions or patients with open prescriptions.

${ }^{84}$ For 2014 and 2015, we observe slightly greater numbers of buprenorphine prescribers than are given in the official state data - by one prescriber and four prescribers, respectively. Since providers without waivers should not show up as buprenorphine prescribers in our data set, this discrepancy most likely owes to our location data - that is, some providers who report Rhode Island Zip codes might not have been identified as Rhode Island-based practitioners in the official Rhode Island counts of waivered providers.
} 
2015 all waivered providers were active prescribers to insured patients, but that in 2016 and 2017 significant numbers of waivered providers either wrote no buprenorphine prescriptions or wrote prescriptions only to patients who paid out of pocket for the drug or who were enrolled in plans that are exempt from reporting to HealthFacts RI. ${ }^{85}$ Based on any of those reasons, access to buprenorphine might be less than what is indicated by the official tally of waivered prescribers. ${ }^{86}$

Separate research (Flavin et al. 2020) finds that of the prescribers listed in SAMHSA's buprenorphine treatment locator in 2019 and practicing in any of 10 states including Rhode Island, 30 percent could not be reached by phone after repeated attempts, and more than onequarter of those that were contacted had stopped prescribing buprenorphine. The same study finds that large shares of active providers do not accept either Medicaid or private health insurance. ${ }^{87}$ These independent findings suggest that the apparent inactivity of buprenorphine providers in our sample is not merely a shortcoming of the data.

\section{Discussion}

This paper is the first to use the HealthFacts RI database to analyze treatment patterns and outcomes related to opioid use disorder, and it belongs to a relatively small group of studies using large administrative panel data sets to analyze similar issues. Our results indicate that individuals adhering recently to medication-assisted treatment (MAT) experience significant reductions in their risk of having a second opioid overdose following an initial (nonfatal) overdose. Although it is possible that the association is not causal, the finding appears to support the effectiveness of MAT in Rhode Island, and it agrees with similar findings from previous studies that use data from other geographic contexts (Larochelle et al. 2018; Pierce et al. 2015). Furthermore, the results are consistent with a much wider body of research, including

\footnotetext{
${ }^{85}$ Because some plans observed before 2016 became exempt in late 2015, the decreased coverage of HealthFacts RI relative to insured Rhode Islanders may account for some of the apparent inactivity of buprenorphine prescribers in our data beginning in 2016. However, these were mostly medical plans, not pharmacy plans, and patients with exempt medical plans continue to exhibit pharmacy claims beyond 2016.

${ }^{86}$ Nearly all of the prescribers in our data set who appeared to cease prescribing buprenorphine beyond a certain date nonetheless continued to prescribe other medications within the succeeding year. Cessation of buprenorphine prescribing should not reflect expiration of waivers, as a waiver remains valid as long as the physician is in compliance with its terms. However, prescribers approved to treat as many as 275 patients with buprenorphine need to renew that elevated patient limit every three years or revert to a lower patient limit.

${ }^{87}$ In addition to Flavin et al. 2020, see also Lila Flavin and J. Wesley Boyd, "A Government Database Is Supposed to Help People Seeking Treatment for Opioid Addiction. It Often Doesn’t,” STAT News, January 9, 2020.
} 
randomized clinical trials, indicating that MAT promotes abstinence from illicit opioid use and reduces other health risks such as HIV.

The results offer support for the policy recommendation that MAT be initiated immediately following an opioid overdose, in the hospital if possible, because emergency department visits may be the only point of medical care for some patients (see Jaeger and Fuehrlein 2020). In 2019, Massachusetts became the first state in the country to require hospitals to offer MAT to patients admitted to the emergency room for overdose and other complications of opioid use disorder (OUD) ${ }^{88}$ Outside of Massachusetts the policy has been adopted on a hospital-byhospital basis, and it is estimated that only 27 percent of hospitals nationwide offer this option. Although Rhode Island does not require hospitals to offer MAT immediately after an overdose, in 2016 the state passed a law (the first of its kind in the United States) that requires that all patients arriving at a hospital due to an overdose be provided with a discharge plan that includes referrals to providers offering MAT. ${ }^{89}$

Our findings also emphasize the need to help patients stay on medications for OUD rather than just helping them to initiate treatment, as merely having taken MAT ever (as opposed to recently) had no protective effect against overdose. Analyzing MAT durations in the sample of patients diagnosed with opioid dependence who ever received MAT, we find that the typical treatment duration complies with medical recommendations suggesting at least 12 to 15 months on MAT; nonetheless, a large share of patients tended to stay on MAT for fewer than 12 months at a time. Policies that have been proposed to facilitate retention in methadone treatment in particular include relaxing restrictions on take-home doses, deploying mobile methadone vans, and establishing "medication-only" sites. ${ }^{90}$

Our analysis reveals a number of apparent disparities in access to and/or uptake of MAT for OUD. Within the sample used to analyze the receipt of MAT, patients with Medicaid insurance had a much greater chance of receiving methadone treatment than patients with non-Medicaid

\footnotetext{
${ }^{88}$ See Martha Bebinger, "Now Mandated to Offer Meds for Opioid Addiction in the ER, Mass. Hospitals Get 'HowTo’ Guidelines," WBUR, January 7, 2019.

${ }^{89}$ For details, see "Levels of Care for Rhode Island Emergency Departments and Hospitals for Treating Overdose and Opioid Use Disorder," Rhode Island Department of Health, Department of Behavioral Healthcare, Developmental Disabilities and Hospitals.

${ }^{90}$ See Alaina McBournie, Alexandra Duncan, Elizabeth Connolly, and Josh Rising, "Methadone Barriers Persist, Despite Decades of Evidence," Health Affairs Blog, September 23, 2019.
} 
plans. This finding agrees with previous evidence that Medicaid promotes increased access to MAT among OUD patients (see, for example, Clemans-Cope et al. 2017) and supports federal policies that would incentivize the expansion of Medicaid in states that have not yet done so. At the same time, Medicaid patients in our sample were not more likely to be treated with buprenorphine compared with non-Medicaid patients. This set of outcomes requires further investigation, as it suggests that among such patients access to buprenorphine may be limited relative to methadone access. If reimbursement rates discourage buprenorphine providers from accepting Medicaid patients, as has been suggested based on a national study (Knudsen and Studts 2019), a review of such rates would be an important subject for policy debate.

We find that alcohol use disorder (AUD) is highly prevalent among OUD patients and carries a heightened risk of a second opioid overdose for patients who have had a first overdose. Despite these facts, OUD patients with comorbid AUD are significantly less likely to receive methadone (but not buprenorphine). Therefore, identifying best practices for the coordinated treatment of OUD and AUD should be an urgent concern. The shortfall in the MAT rate (for either methadone or buprenorphine) among women compared with men, although modest, also deserves attention. The reasons for this disparity are not readily apparent, as the analysis controls for numerous comorbidities and other factors, and our model of repeated overdose indicates that women face higher cumulative risks of a repeated overdose than otherwise similar men within the first four years after an initial overdose.

Geography may also differentially affect access to medications for OUD, as we find that living in a high-poverty Zip code is associated with a higher chance of receiving methadone and a lower chance of receiving buprenorphine. Previous research identifies disparities in the supply of buprenorphine providers between urban and rural areas (Andrilla et al. 2018), although we find no association between living in a rural area and being treated with either methadone or buprenorphine. However, information on the practice locations of buprenorphine providers is known to be inaccurate (Flavin et al. 2020). ${ }^{91}$ Accordingly, policymakers might work to enhance the accuracy of treatment location information so that they can better assess which communities

\footnotetext{
${ }^{91}$ See also Lila Flavin and J. Wesley Boyd, “A Government Database Is Supposed to Help People Seeking Treatment for Opioid Addiction. It Often Doesn't,” STAT News, January 9, 2020.
} 
are underserved and help patients connect with active prescribers that are located close to where they live.

Our analysis of changes in buprenorphine prescribing practices following federal policy changes enacted in 2016 supports the view that raising patient limits can enable select prescribers to serve more patients and as a result expand the total patient pool. However, further increases in patient limits should be less effective at the margin, as it is unlikely that an individual prescriber can accommodate more than 275 buprenorphine patients at a time. The analysis also finds that the CARA act of 2016, by allowing nurse practitioners and physician assistants to prescribe buprenorphine, may have helped more Rhode Island patients in high-poverty Zip codes gain access to the drug. This latter finding offers support to proposed policies that would further expand the pool of buprenorphine prescribers, such as one that would empower pharmacists to obtain waivers to prescribe the medication and a more sweeping change that would remove the waiver requirement altogether. Without the waiver requirement, practitioners in eligible groups could get permission to prescribe buprenorphine simply by verifying their professional credentials, rather than undertaking extra training and submitting a lengthy application. ${ }^{92}$

A key concern revealed by the analysis is that as of 2017, the typical prescriber of buprenorphine in Rhode Island served an estimated 14 patients at a given time--less than half of the 30-patient limit afforded to most practitioners and well below the higher patient limits that practitioners can eventually obtain. Research on other states also finds that most buprenorphine providers have caseloads far below their patient limits (Thomas et al. 2017), a situation that points to the reported challenges faced by non-specialist practitioners in treating OUD patients, who tend to have complex medical conditions (Knudsen et al. 2011; Netherland et al. 2009). Vermont's huband-spoke treatment model for OUD, which links office-based practitioners (spokes) with support services from specialized opioid treatment programs (hubs), has proven effective at increasing patient density per buprenorphine prescriber (Brooklyn and Sigmon 2017). Accordingly, other states might do more to emulate that model. Rhode Island has opioid

\footnotetext{
${ }^{92}$ See UMass School of Medicine Specialists, "Remove the Waiver Requirement for Buprenorphine Prescribing," Boston Globe, February 22, 2021, https://www.bostonglobe.com/2021/02/22/opinion/remove-waiver-requirementbuprenorphine-prescribing/.
} 
treatment programs that resemble hubs, but not every practitioner in Rhode Island is formally tied with a hub.

Various other policies to promote greater access to MAT have been advocated in recent years, such as expanding telemedicine for the delivery of MAT and relaxing restrictions on take-home doses of methadone. Both of these policies received a boost as a result of the COVID-19 pandemic. In April 2020 the federal government suspended a law against telemedicine delivery of MAT, and patients in Rhode Island (and many other states) can initiate buprenorphine treatment through an audio-only call as long as the COVID-19 public health emergency remains in effect. ${ }^{93}$ Telehealth-delivered MAT was found to increase treatment retention in small-scale studies conducted before the pandemic (Ho and Argáez 2018), and the current experiment should offer opportunities to assess the approach on a broader scale, potentially leading to permanent changes in policy. The pandemic also occasioned a temporary relaxation of rules governing the number of take-home doses of methadone and buprenorphine, and patient advocates are keen to see if the more liberal policies can achieve increased treatment retention without resulting in greater misuse and diversion (del Pozo and Rich 2020). Also in response to the pandemic, several states (including Rhode Island) and the federal government took temporary measures to ensure access to health insurance regardless of employment status, such as extending the open enrollment periods for non-employer-based insurance plans. ${ }^{94}$

Rhode Island has made much progress in helping a large share of patients to access MAT for the treatment of OUD. At the same time the state continues to struggle with elevated overdose rates. Although this combination of facts might seem discouraging, our findings suggest that in Rhode Island, MAT appears to be working to significantly reduce overdose risk, provided patients have

\footnotetext{
${ }^{93}$ Prior to the pandemic (June 2019) one of the largest providers of MAT in Rhode Island began offering the option to initiate buprenorphine treatment via a video call. See G. Wayne Miller, "Program Offers Teleconferencing to Treat Substance-abuse Disorders," Providence Journal, June 24, 2019. For information on the federal directive, see "Electronic Prescriptions for Controlled Substances," US Department of Justice, Drug Enforcement Administration, Diversion Control Division.

${ }^{94}$ See "When Can I Enroll in Private Health Plan Coverage through the Marketplace?" Kaiser Family Foundation, https://www.kff.org/faqs/faqs-health-insurance-marketplace-and-the-aca/when-can-i-enroll-in-private-health-plancoverage-through-the-marketplace/; "Extension of Certain Timeframes for Employee Benefit Plans, Participants, and Beneficiaries Affected by the COVID-19 Outbreak," The Federal Register, May 4, 2020 , https://www.federalregister.gov/documents/2020/05/04/2020-09399/extension-of-certain-timeframes-for-employeebenefit-plans-participants-and-beneficiaries-affected; and Tami Luhby, "Biden Signs Executive Order to Reopen Affordable Care Act Enrollment," CNN, January 28, 2021, https://www.cnn.com/2021/01/28/politics/bidenexecutive-orders-health-care-aca-medicaid/index.html.
} 
taken the medications recently. In sum, the evidence argues strongly for policies that would focus on improved treatment retention and not just initiation. 


\section{References}

American Psychiatric Association. 2013. Diagnostic and Statistical Manual of Mental Disorders (5th ed.). Washington, DC.

American Society of Addiction Medicine. 2017. "Billing and Coding: Medication-assisted Treatment." American Society of Addiction Medicine.

American Society of Addiction Medicine. 2018. "Public Policy Statement on Prescription Drug Monitoring Programs (PDMPs)" American Society of Addiction Medicine. April 11, 2018.

Anderson, Tammy L., and Judith A. Levy. 2003. "Marginality among Older Injectors in Today's Illicit Drug Culture: Assessing the Impact of Ageing.” Addiction 98: 761-770.

Barocas, Joshua A., Laura F. White, Jianing Wang, Alexander Y. Walley, Marc R. LaRochelle, Dana Bernson, Thomas Land, Jake R. Morgan, Jeffrey H. Samet, and Benjamin P. Linas, 2018. "Estimated Prevalence of Opioid Use Disorder in Massachusetts, 20112015: A Capture-Recapture Analysis.” American Journal of Public Health 108: 16751681. https://doi.org/10.2105/AJPH.2018.304673.

Brooklyn, John R., and Stacey C. Sigmon. 2017. "Vermont Hub-and-Spoke Model of Care for Opioid Use Disorder: Development, Implementation, and Impact.” Journal of Addiction Medicine 11(4): 286-292. https://doi.org/10.1097/ADM.0000000000000310

Burke, Mary A. 2019. “Access to Medication-assisted Treatment for Opioid Use Disorder: Is Rhode Island Different, and Why?" Federal Reserve Bank of Boston Current Policy Perspectives No. 19-2.

Choo, Christie. 2009. "Medications Used in Opioid Maintenance Treatment." US Pharmacist 34(11): 40-53.

Clemans-Cope, Lisa, Victoria Lynch, Marni Epstein, and Genevieve Kenney. 2017. "Medicaid Coverage of Effective Treatment for Opioid Use Disorder: Trends in State Buprenorphine Prescriptions and Spending since 2011.” Urban Institute Research Report. June 8, 2017.

Connery, Hilary Smith MD. 2015. "Medication-assisted Treatment of Opioid Use Disorder." Harvard Review of Psychiatry 23(2): 63-75. 10.1097/HRP.0000000000000075

Conner, Kyaien O., and Daniel Rosen. 2008. “You're Nothing But a Junkie': The Multiple Experiences of Stigma in an Aging Methadone Maintenance Population." Journal of Social Work Practice in the Addictions 8: 244-264. https://doi.org/10.1080/15332560802157065

del Pozo, Bandon, and Josiah D. Rich. 2020. "Revising Our Attitudes towards Agonist Medications and Their Diversion in a Time of Pandemic." Journal of Substance Abuse Treatment 119. https://doi.org/10.1016/j.jsat.2020.108139

del Pozo, Brandon, Lawrence S. Krasner, and Sarah F. George. 2020. "Decriminalization of Diverted Buprenorphine in Burlington, Vermont, and Philadelphia: An Intervention to 
Reduce Opioid Overdose Deaths." The Journal of Law, Medicine \& Ethics 48(2): 373375. https://doi.org/10.1177/1073110520935353

Department of Health and Human Services. 2001. "Opioid Drugs in Maintenance and Detoxification Treatment of Opiate Addiction.” Federal Register 66: 2076-4102. Accessed October 18, 2019.

Diaper, A. M., F. D. Law, and J. K. Melichar. 2014. "Pharmacological Strategies for Detoxification.” British Journal of Clinical Pharmacology 77(2): 302-314.

Evans, Elizabeth A., Yuhui Zhu, Caroline Yoo, David Huang, and Yih-Ing Hser. 2019. "Criminal Justice Outcomes over 5 Years after Randomization to BuprenorphineNaloxone or Methadone Treatment for Opioid Use Disorder." Addiction 114: 13961404. https://doi.org/10.1111/add.14620

Faul, Mark, Michele Bohm, and Caleb Alexander. 2017. "Methadone Prescribing and Overdose and the Association with Medicaid Preferred Drug List Policies-United States, 20072014." MMWR Morb Mortal Wkly Rep 2017(66): 320-323. http://dx.doi.org/10.15585/mmwr.mm6612a2external icon

Flavin, Lila, Monica Malowney, Nikhil A. Patel, Michael D. Alpert, Elisa Cheng, Gaddy Do Noy, Sarah Samuelson, Nina Sreshta, and J. Wesley Boyd. 2020. "Availability of Buprenorphine Treatment in the 10 States with the Highest Drug Overdose Death Rates in the United States." Journal of Psychiatric Practice 26(1): 17- 22. 10.1097/PRA.0000000000000437

Gibson, Amy, Louisa Degenhardt, Richard P. Mattick, Robert Ali, Jason White, and Susannah O’Brien. 2008. "Exposure to Opioid Maintenance Treatment Reduces Long-term Mortality. Addiction 103(3): 462-468. https://doi.org/10.1111/j.1360-0443.2007.02090.x

Green, Traci C., Jennifer Clarke, Lauren Brinkley-Rubinstein, Brandon D. L. Marshall, Nicole Alexander-Scott, Rebecca Boss, and Josiah D. Rich. 2018. "Postincarceration Fatal Overdoses after Implementing Medications for Addiction Treatment in a Statewide Correctional System.” JAMA Psychiatry 75(4): 405-407.

Grimm, Christi A. 2020. "Geographic Disparities Affect Access to Buprenorphine Services for Opioid Use Disorder," U.S. Department of Health and Human Services, Office of Inspector General. January 2020.

Gudin, Jeffrey A., Shanthi Mogali, Jermaine D. Jones, and Sandra D. Comer. 2013. "Risks, Management, and Monitoring of Combination Opioid, Benzodiazepines, and/or Alcohol Use." Postgraduate Medicine 125(4): 115-130. https://doi.org/10.3810/pgm.2013.07.2684

Ho, Chuong, and Charlene Argáez. 2018. "Telehealth-delivered Opioid Agonist Therapy for the Treatment of Adults with Opioid Use Disorder: Review of Clinical Effectiveness, Costeffectiveness, and Guidelines." Canadian Agency for Drugs and Technologies in Health. https://www.ncbi.nlm.nih.gov/books/NBK537877/

Institute of Medicine. 1995. Federal Regulation of Methadone Treatment. Washington, DC: The National Academies Press. https://doi.org/10.17226/4899 
Jaeger, Stephen, and Brian Fuehrlein. 2020. "Buprenorphine Initiation to Treat Opioid Use Disorder in Emergency Rooms." Journal of the Neurological Sciences 411. https://doi.org/10.1016/j.jns.2020.116716

Jarvis, Brantley P., August F. Holtyn, Shrinidhi Subramaniam, D. Andrew Tompkins, Emmanuel A. Oga, George E. Bigelow, and Kenneth Silverman. 2018. "Extended-release Injectable Naltrexone for Opioid Use Disorder: A Systematic Review.” Addiction 113(7): 11881209. https://doi.org/10.1111/add.14180

Johnson, Quentin, Brian Mund, and Paul J. Joudrey. 2018. "Improving Rural Access to Opioid Treatment Programs.” Journal of Law, Medicine \& Ethics 46(2): 437-439. https://doi.org/10.1177/1073110518782951

Jones, Christopher M., Melinda Campopiano, Grant Baldwin, and Elinore McCance-Katz. 2015. "National and State Treatment Need and Capacity for Opioid Agonist Medicationassisted Treatment." American Journal of Public Health 105(8): 55-63.

Kaiser Family Foundation. 2020. “Opioid Overdose Death Rates and All Drug Overdose Death Rates per 100,000 Population (Age-Adjusted).” Kaiser Family Foundation. February 13, 2020.

Kelty, Erin, and Gary Hulse. 2017. "Fatal and Non-fatal Opioid Overdose in Opioid Dependent Patients Treated with Methadone, Buprenorphine or Implant Naltrexone." The International Journal on Drug Policy 46: 54-60. https://doi.org/10.1016/j.drugpo.2017.05.039

Krantz, Mori J., and Philip S. Mehler. 2004. "Treating Opioid Dependence: Growing Implications for Primary Care.” Archives of Internal Medicine 164(3): 277-288. https://doi.org/10.1001/archinte.164.3.277

Knudsen, Hannah K., Paul M. Roman, and Carrie B. Oser. 2011. "Facilitating Factors and Barriers to the Use of Medications in Publicly Funded Addiction Treatment Organizations," Journal of Addiction Medicine 4(2): 99-107. https://doi.org/10.1097/ADM.0b013e3181b41a32

Knudsen, Hannah K., and Jamie L. Studts. 2019. "Physicians as Mediators of Health Policy: Acceptance of Medicaid in the Context of Buprenorphine Treatment." The Journal of Behavioral Health Services and Research 46(1): 151-163. https://doi.org/10.1007/s11414-018-9629-4

Larochelle, Marc R., Dana Bernson, Thomas Land, Thomas J. Stopka, Na Wang, Ziming Xuan, Sarah M. Bagley, Jane M. Liebschutz, and Alexander Y. Walley. 2018. "Medication for Opioid Use Disorder after Nonfatal Opioid Overdose and Association with Mortality." Annals of Internal Medicine 169(3): 137-145. https://doi.org/10.7326/M17-3107

Lee J.D., E.V. Nunes, P Novo, et al. 2018. “Comparative Effectiveness of Extended-release Naltrexone Versus Buprenorphine-naloxone for Opioid Relapse Rrevention (X:BOT): A Multicentre, Open-label, Randomised Controlled Trial.” Lancet 391(10118): 309-318. https://doi.org/10.1016/S0140-6736(17)32812-X

Ma, Jun, Yan-Ping Bao, Ru-Jia Wang, Meng-Fan Su, Mo-Xuan Liu, Jin-Qiao Li, Louisa Degenhardt, Michael Farrell, Frederic C. Blow, Mark Ilgen, Jie Shi, and Lin Lu. 
2018. "Effects of Medication-assisted Treatment on Mortality among Opioids Users: A Systematic Review and Meta-analysis" Molecular Psychiatry 24(12): 18681883. https://doi.org/10.1038/s41380-018-0094-5

Maclean, Johanna Catherine, and Brendan Saloner. 2017. "The Effect of Public Insurance Expansions on Substance Use Disorder Treatment: Evidence from the Affordable Care Act.” NBER Working Paper 23342.

Manchester, Joyce, and Riley Sullivan. 2019. "Exploring Causes of and Responses to the Opioid Epidemic in New England.” New England Public Policy Center Policy Reports 19-2. Federal Reserve Bank of Boston.

McBain, Ryan K., Andrew Dick, Mark Sorbero, and Bradley D. Stein. 2020. "Growth and Distribution of Buprenorphine-waivered Providers in the United States, 2007-2017." Annals of Internal Medicine 172(7): 504-506. https://doi.org/10.7326/M19-2403

Mohlman, Mary Kate, Beth Tanzman, Karl Finison, Melanie Pinette, and Craig Jones. 2016. "Impact of Medication-assisted Treatment for Opioid Addiction on Medicaid Expenditures and Health Services Utilization in Vermont." Journal of Substance Abuse 67: 9-14. https://doi.org/10.1016/j.jsat.2016.05.002

National Academies of Sciences, Engineering, and Medicine. 2019. Medications for Opioid Use Disorder Save Lives. Washington, DC: The National Academies Press. https://doi.org/10.17226/25310

National Institute on Drug Abuse. 2020. "How Effective Are Medications to Treat Opioid Use Disorder?” National Institute on Drug Abuse Research Report. June 17, 2020.

National Institute on Drug Abuse. 2018. "Principles of Drug Addiction Treatment: A ResearchBased Guide (Third Edition).” National Institute on Drug Abuse, National Institutes of Health, Department of Health and Human Services.

Netherland, Julie, Michael Botsko, James E. Egan, Andrew J. Saxon, Chinazo O. Cunningham, Ruth Finkelstein, Mark N. Gourevitch, John A. Renner, Nancy Sohler, Lynn E. Sullivan, Linda Weiss, and David A. Fiellin. 2009. "Factors Affecting Willingness to Provide Buprenorphine Treatment." Journal of Substance Abuse Treatment 36(3): 244-251. https://doi.org/10.1016/j.jsat.2008.06.006

Neighbors, Charles J., Sugy Choi, Shannon Healy, Rajeev Yerneni, Tong Sun, and Liudmila Shapoval. 2019. "Age Related Medication for Addiction Treatment (MAT) Use for Opioid Use Disorder among Medicaid-insured Patients in New York.".Substance Abuse Treatment, Prevention, and Policy 14: article number 28. https://doi.org/10.1186/s13011019-0215-4

Pierce, Mathias, Sheila M. Bird, Matthew Hickman, John Marsden, Graham Dunn, Andrew Jones, and Tim Millar. 2015. "Impact of Treatment for Opioid Dependence on Fatal Drug-related Poisoning: A National Cohort Study in England.” Addiction 111: 298308. 10.1111/add.13193

Pullen, Ellen, and Carrie Oser. 2014. "Barriers to Substance Abuse Treatment in Rural and Urban Communities: A Counselor Perspective," Substance Use \& Misuse 49(7): 891901. https://dx.doi.org/10.3109/10826084.2014.891615 
Rough, Kathryn, Brian T. Bateman, Elisabeth Patorno, Rish J. Desai, Younyoung Park, Sonia Hernandez-Diaz, Krista F. Huybrechts. 2016. "Suppression of Substance Abuse Claims in Medicaid Data and Rates of Diagnoses for Non-Substance Abuse Conditions." JAMA 315(11): 1164-1166. doi:10.1001/jama.2015.18417

Saloner, Brendan and Shankar Karthikeyan. 2015. "Changes in Substance Abuse Treatment Use among Individuals with Opioid Use Disorders in the United States, 2004-2013." JAMA 314(14): 1515-1517. https://doi.org/10.1001/jama.2015.10345

Shapiro, Aaron, Lisa R. Villarroel, and Paul George. 2019. "A Call to Maximize Impact of the SUPPORT for Patients and Communities Act through Standard Inclusion of Opioid Use Disorder Treatment Curricula in Medical Schools." Advances in Medical Education and Practice 10: 581-583. 10.2147/AMEP.S205946

Sordo, Luis, Gregorio Barrio, Maria J Bravo, B. Iciar Indave, Louisa Degenhardt, Lucas Wiessing, Marica Ferri, and Roberto Pastor-Bariusso. 2017. "Mortality Risk during and after Opioid Substitution Treatment: Systematic Review and Meta-analysis of Cohort Studies." The BMJ 357: j1550. 10.1136/bmj.j1550

Stein, Bradley D., Mark Sorbero, Andrew W. Dick, Rosalie Liccardo Pacula, Rachel M. Burns, and Adam J. Gordon. 2016. "Physician Capacity to Treat Opioid Use Disorder with Buprenorphine-Assisted Treatment.” JAMA 316(11): 1211-1212.

Thomas, Cindy Parks, Erin Doyle, Peter W. Kreiner, Christopher M. Jones, Joel Dubenitz, Alexis Horan, and Bradley D. Stein. 2017. "Prescribing Patterns of Buprenorphine Waivered Physicians." Drug and Alcohol Dependence 181: 213-218. https://doi.org/10.1016/j.drugalcdep.2017.10.002

Volkow, Nora D., George F. Koob, and A. Thomas McLellan. 2016. "Neurobiologic Advances from Brain Disease Model of Addiction." The New England Journal of Medicine 374: $363-371$.

Walley, Alexander Y., Debbie Cheng, C.E. Pierce, Clara Chen, T. Filippell, Jeffrey H. Samet, and D.P. Alford. 2012. "Methadone Dose, Take Home Status, and Hospital Admission among Methadone Maintenance Patients.” Journal of Addiction Medicine 6: 186-190.

Wen, Hefei, Jason M Hockenberry, Tyrone F. Borders, and Benjamin G. Druss. 2017. "Impact of Medicaid Expansion on Medicaid-covered Utilization of Buprenorphine for Opioid Use Disorder Treatment." Medical Care 55(4): 336-341.

Wen, Hefei, Jason M. Hockenberry, and Harold A. Pollack. 2018. "Association of Buprenorphine-waivered Physician Supply with Buprenorphine Treatment Use and Prescription Opioid Use in Medicaid Enrollees." JAMA Network Open 1(5). https://doi.org/10.1001/jamanetworkopen.2018.2943

Williams, Arthur Robin, Hillary Samples, Stephen Crystal, and Mark Olfson. 2020. “Acute Care, Prescription Opioid Use, and Overdose following Discontinuation of Long-Term Buprenorphine Treatment for Opioid Use Disorder." The American Journal of Psychiatry 177(2): 117-124. 10.1176/appi.ajp.2019.19060612 
World Health Organization. 2009. "Guidelines for the Psychosocially Assisted Pharmacological Treatment of Opioid Dependence." 
Figure 1: Age-adjusted Opioid Overdose Mortality Rates per 100,000 Persons, 1999-2018

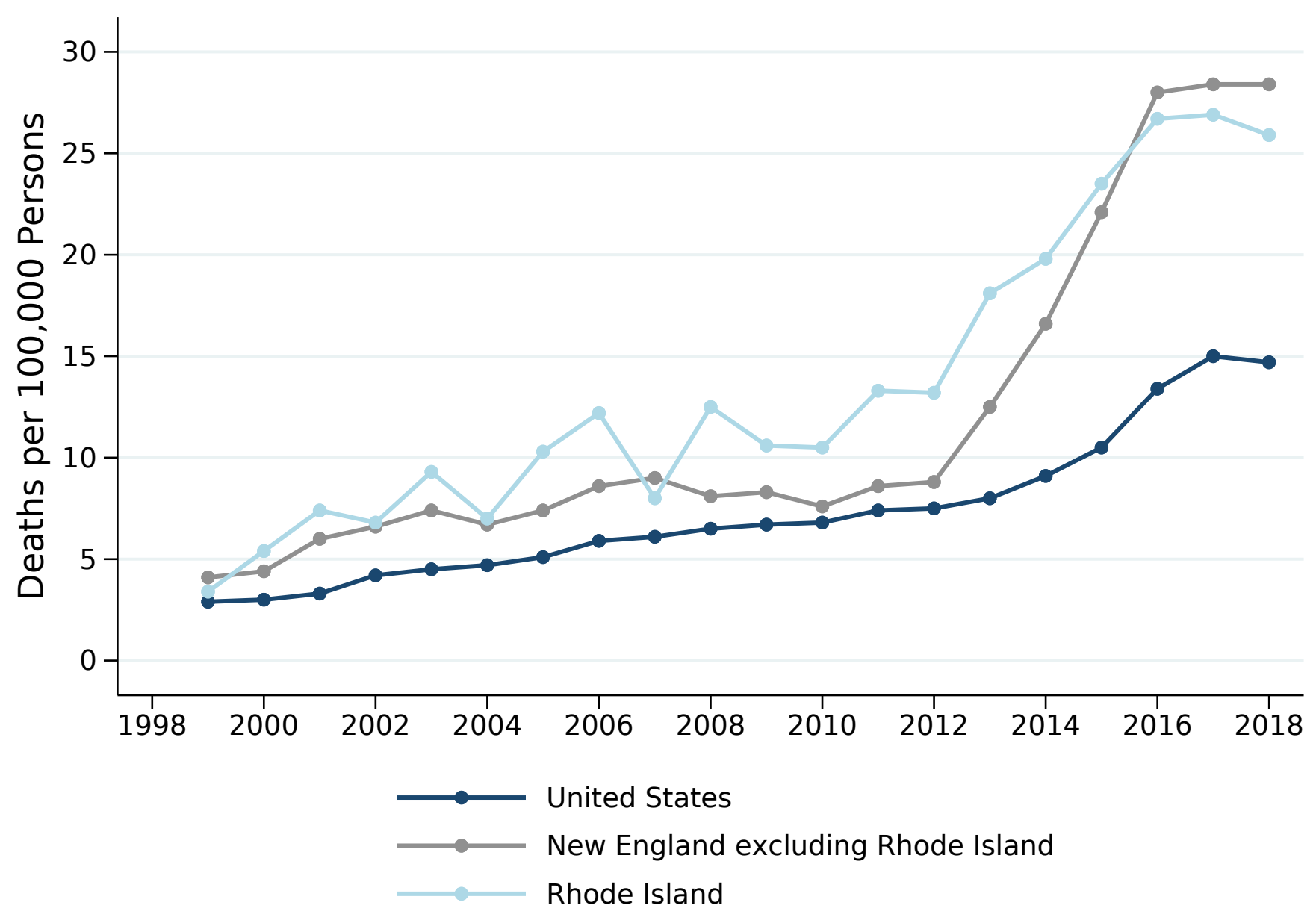

Source: Centers for Disease Control and Prevention.

Notes: The base population in each geographic area includes all residents. Values for New England excluding Rhode Island are population-weighted average mortality rates per year among Connecticut, Maine, Massachusetts, New Hampshire, and Vermont. 
Figure 2: Average Monthly HealthFacts RI Enrollees vs. All Rhode Island Residents

Fiscal Years 2012-2018

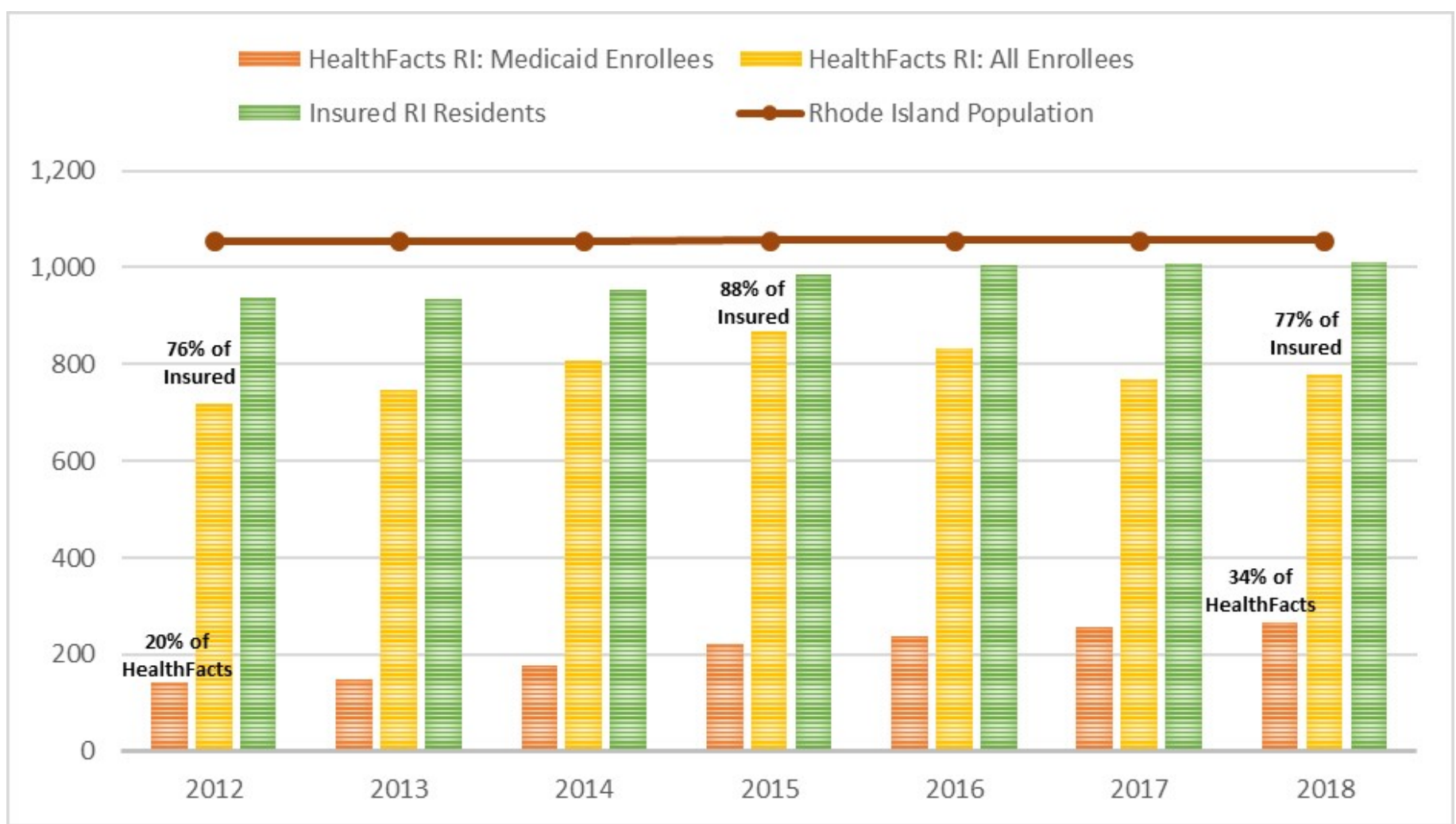

Sources: Authors' calculations using data from HealthFacts RI, US Census Bureau/Haver Analytics, and American Community Survey.

Notes: All numbers are in thousands of individuals. Values represent actual or estimated monthly averages for the given fiscal year of the number of individuals in each population or subgroup. HealthFacts RI Medicaid enrollees exclude those with both Medicaid and Medicare insurance. The number of insured Rhode Island residents was calculated as the percentage of Rhode Islanders with health insurance (estimated for the fiscal year based on the American Community Survey) times the monthly average RI population in the fiscal year. 


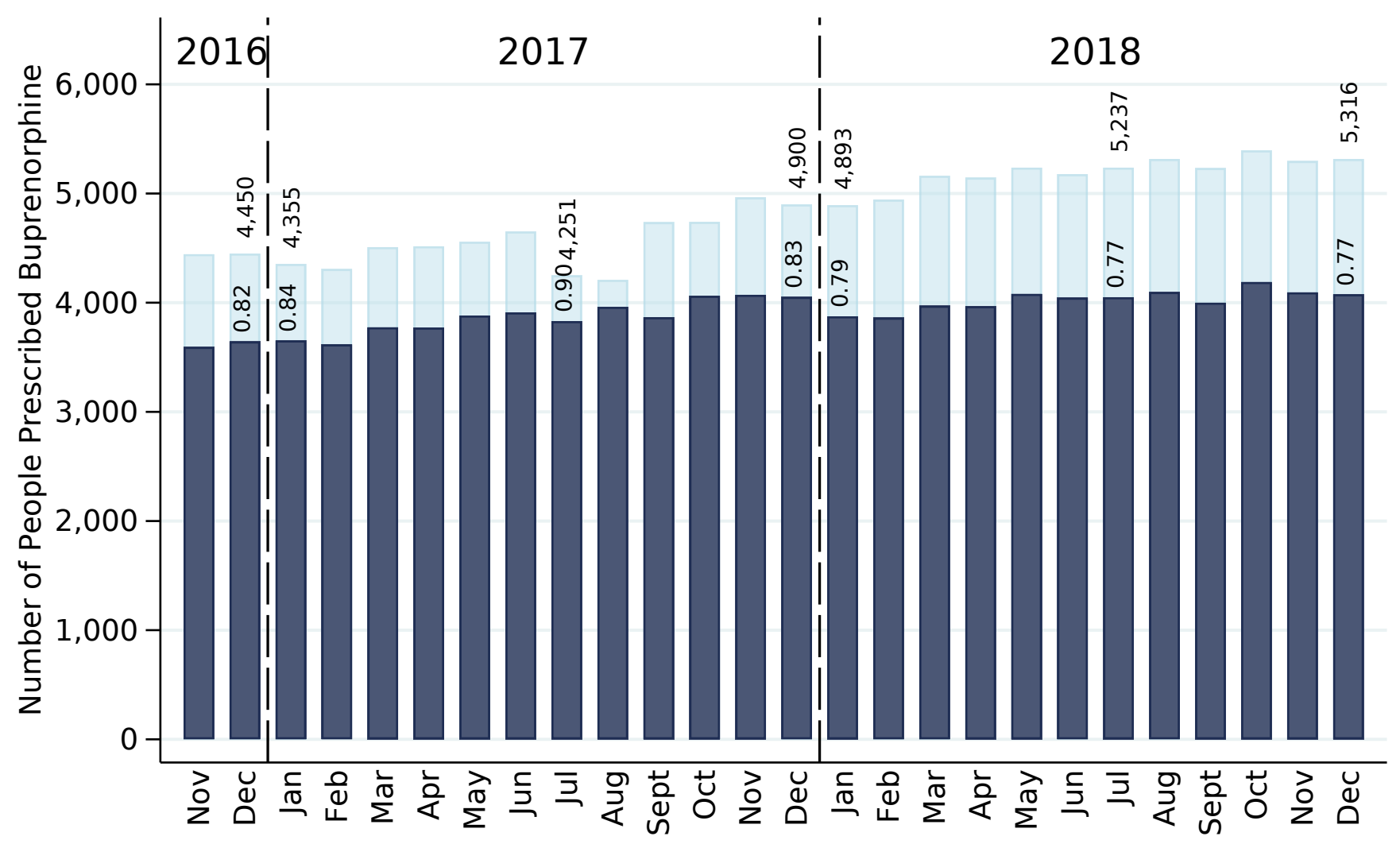

All RI Buprenorphine Patients

HealthFacts RI Buprenorphine Patients

Sources: Authors' calculations using HealthFacts RI and Prevent Overdose RI.

Notes: "HealthFacts RI buprenorphine patients" refers to the number of unique buprenorphine recipients in the month (restricted to patients aged 18 and older with RI Zip codes and non-denied pharmacy claims). "All RI buprenorphine patients" refers to the number of buprenorphine recipients as reported on the Prevent Overdose RI website. The numbers reported on that website draw on data from the Rhode Island Department of Health (RIDOH), Substance Abuse and Mental Health Services Administration (SAMHSA), and Rhode Island Department of Behavioral Healthcare, Developmental Disabilities and Hospitals (BHDDH). The numbers placed above selected HealthFacts RI bars present the number of buprenorphine patients captured by HealthFacts RI as a share of all Rhode Island buprenorphine patients. 


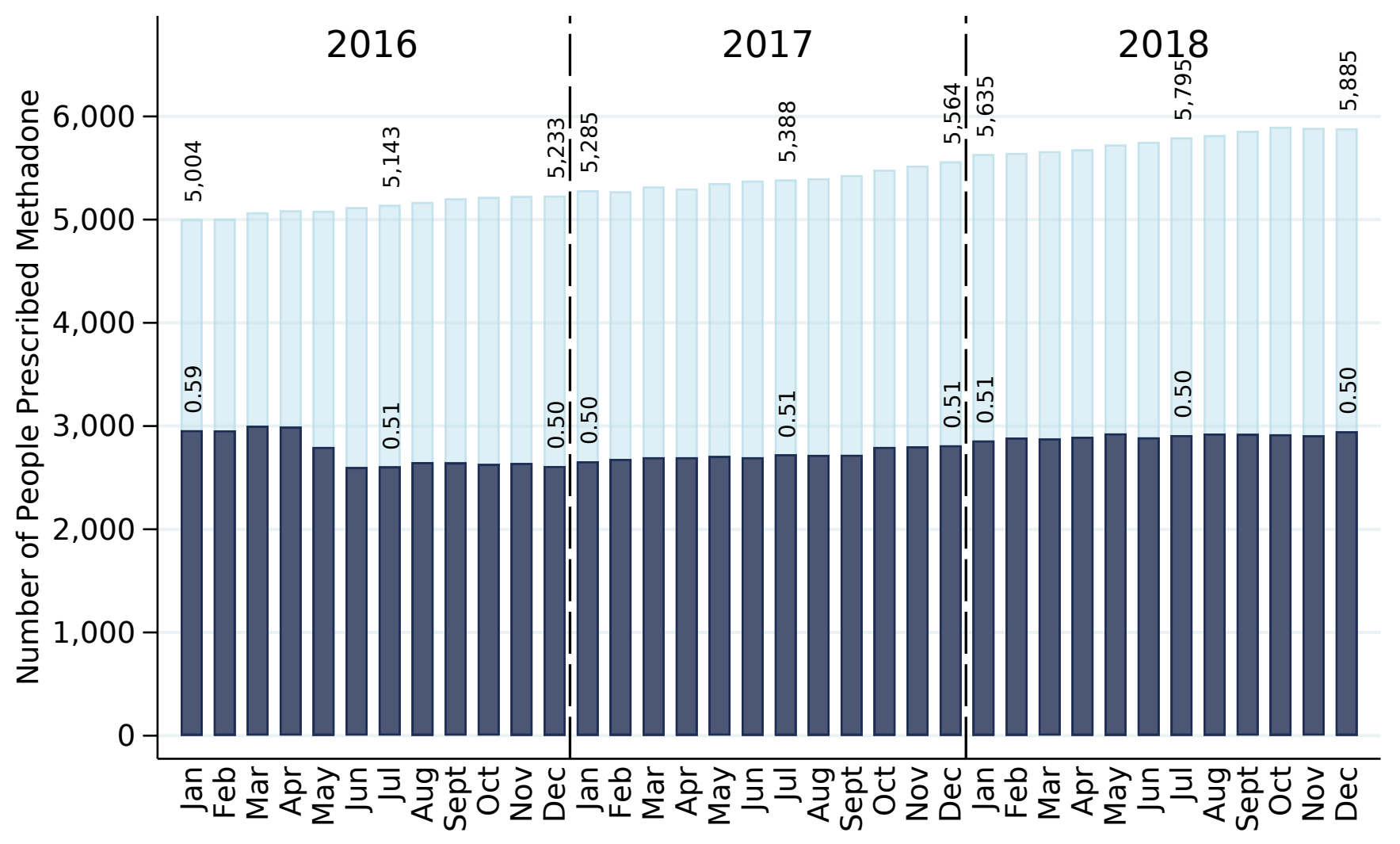

\section{All RI Methadone Patients \\ HealthFacts RI Methadone Patients}

Sources: Authors' calculations using HealthFacts RI and Prevent Overdose RI.

Notes: "HealthFacts RI methadone patients" refers to the number of unique methadone treament recipients in the month (restricted to patients aged 18 and older with RI Zip codes). "All RI methadone patients" refers to the number of methadone treatment recipients as reported on the Prevent Overdose RI website. The numbers reported on that website draw on data from the Rhode Island Department of Health (RIDOH), Substance Abuse and Mental Health Services Administration (SAMHSA), and Rhode Island Department of Behavioral Healthcare, Developmental Disabilities and Hospitals (BHDDH). The numbers placed above selected HealthFacts RI bars present the number of methadone patients captured by HealthFacts $\mathrm{RI}$ as a share of all Rhode Island methadone patients. 


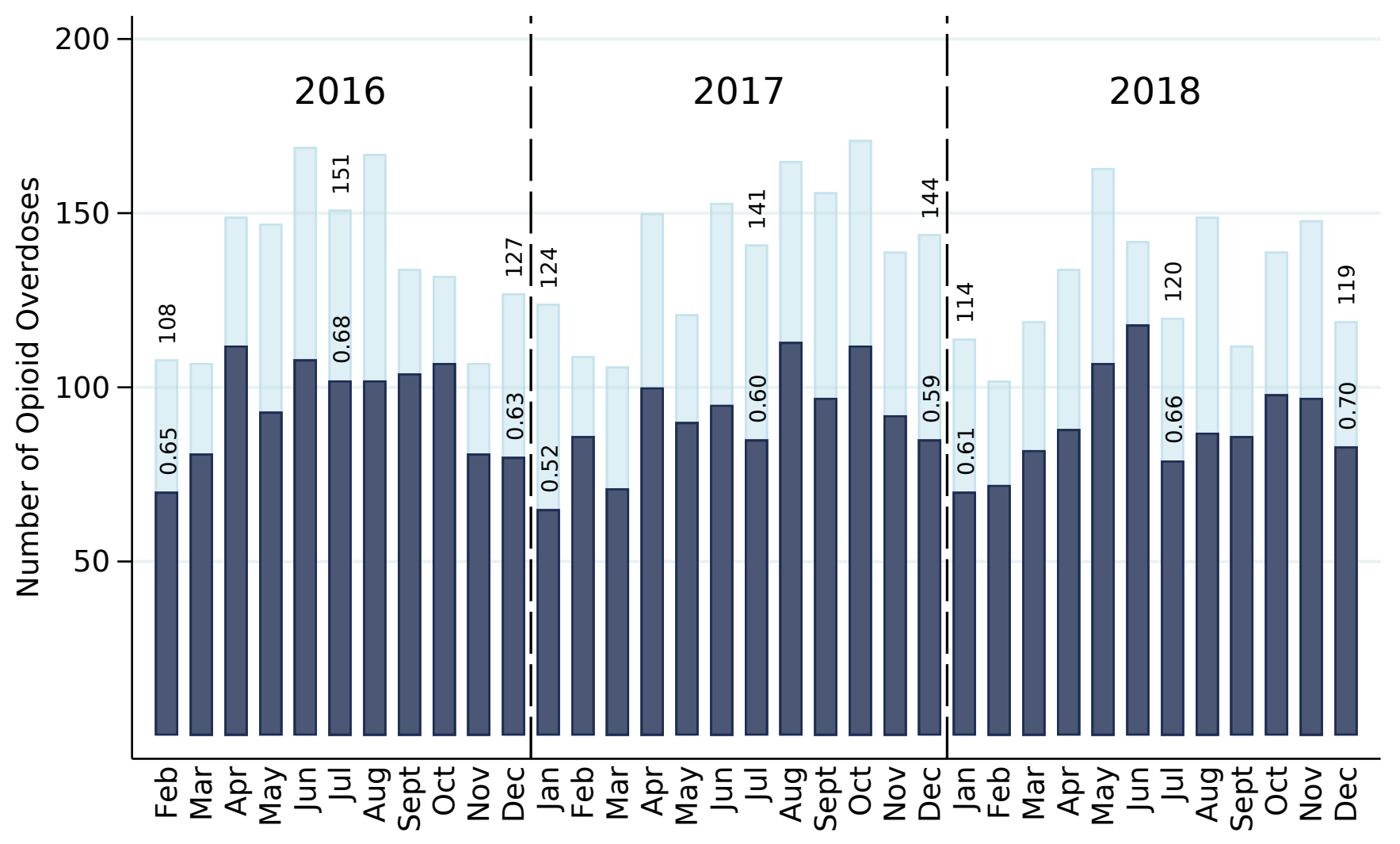

All RI Opioid Overdoses

HealthFacts RI Opioid Overdoses

Sources: Authors' calculations using HealthFacts RI and Prevent Overdose RI.

Notes: "HealthFacts RI opioid overdoses" refers to the number of opioid overdoses in the month (restricted to emergency department visits observed in HealthFacts RI among patients aged 18 and older with RI Zip codes). A given patient may account for more than one overdose in a month provided a given overdose occurs at least two days after the previous one. "All RI opioid overdoses" refers to the number of opioid overdoses per month (restricted to emergency department visits) as reported on the Prevent Overdose RI website. The numbers reported on that website draw on data from the Rhode Island Department of Health (RIDOH), Substance Abuse and Mental Health Services Administration (SAMHSA), and Rhode Island Department of Behavioral Healthcare, Developmental Disabilities and Hospitals (BHDDH). The numbers placed above selected HealthFacts RI bars present the number of opioid overdoses captured by HealthFacts RI as a share of all Rhode Island opioid overdoses. 
Table 1: Percentage of Sample with Selected Characteristics, by Number of Overdoses Nonfatal Opioid Overdose Sample

\begin{tabular}{|c|c|c|c|}
\hline & All & One Overdose & Two or More Overdoses \\
\hline Receives MAT (Ever)* & 47.8 & 41 & 67 \\
\hline Number of Last 3 Months on MAT & 0.5 & 0.5 & 0.6 \\
\hline Starts MAT Before First Overdose & 32.3 & 29.4 & 40.8 \\
\hline Starts MAT After First Overdose & 15.4 & 11.7 & 26.2 \\
\hline Alcohol Use Disorder Diagnosis (Ever) & 55.3 & 50.1 & 70.1 \\
\hline Opioid Dependence Diagnosis (Ever) ${ }^{* *}$ & 68.8 & 63.2 & 85 \\
\hline Opioid Use/Abuse Diagnosis (Ever, At Most) ${ }^{* *}$ & 5.9 & 6.4 & 4.6 \\
\hline Hepatitis C Diagnosis (Ever) & 32.8 & 28.2 & 46 \\
\hline Depression Diagnosis (Ever) & 88.9 & 87.8 & 92.2 \\
\hline Anxiety Diagnosis (Ever) & 88.5 & 86.7 & 93.5 \\
\hline Other Mental Illness Diagnosis (Ever) & 92.8 & 91.5 & 96.5 \\
\hline Other Substance Abuse Disorder Diagnosis (Ever) & 85.1 & 82 & 93.9 \\
\hline Fills Both Opioid and Benzodiazepine Rxs Same Month (Ever) & 52.9 & 52.6 & 53.8 \\
\hline Behavioral Treatment (Ever) & 67.1 & 64.4 & 74.6 \\
\hline First Overdose Age 18-30 & 15 & 12.5 & 22.1 \\
\hline First Overdose Age 31-42 & 23.9 & 22.8 & 26.9 \\
\hline First Overdose Age 43-52 & 23.6 & 23.8 & 23.2 \\
\hline First Overdose Age 53-90 & 37.6 & 41 & 27.8 \\
\hline Male & 53.2 & 52.7 & 54.4 \\
\hline Female & 46.8 & 47.3 & 45.6 \\
\hline Medicaid $^{* * *}$ & 44.1 & 41 & 52.9 \\
\hline Non-Medicaid*** & 55.9 & 59 & 47.1 \\
\hline Enters Sample 2011 & 91.7 & 93 & 88.1 \\
\hline Enters Sample 2012 & 8.3 & 7 & 11.9 \\
\hline Sample Size & 2079 & 1540 & 539 \\
\hline
\end{tabular}

Source: Authors' calculations using HealthFacts RI.

Notes: The term "overdose" always refers to one that involved opioids. In most rows the values represent the percentage of the given group (either the entire sample or the subset with a specific number of overdoses) with the given characteristic. The bottom row shows the number of unique individuals in each group. "Number of last 3 months on MAT" shows the average number of the preceding three months in which an individual either filled a buprenorphine prescription or received methadone maintenance treatment within a given group. Opioid overdoses include those treated in an emergency department or in an inpatient setting. The sample consists of HealthFacts RI enrollees who had at least one nonfatal opioid overdose between April 2011 and May 2019 and at least three months of consecutive observations immediately following their first overdose. These individuals were selected from the larger "incumbent panel," consisting of enrollees who were observed, at a minimum, in all months from January 2013 through December 2015 (excepting as many as three months) and who did not switch between Medicaid and non-Medicaid insurance plans before January 2017. All sample members were at least 18 years of age at the time of their first overdose.

* "Receives MAT (ever)" is defined as receiving methadone maintenance treatment or filling a buprenorphine prescription in at least one month among all of an individual's observations.

** "Opioid use/abuse diagnosis (ever, at most)" is defined as having at least one observation with a diagnosis of either opioid use or opioid abuse, but never having a diagnosis of the more serious condition of opioid dependence. "Opioid dependence diagnosis (ever)" is defined as having at least one observation with a diagnosis of opioid dependence. Other diagnosis-related variables are defined similarly by requiring at least one observation with the given diagnosis. The complete list of ICD-9 and ICD-10 codes used to identify all diagnoses, including opioid overdoses, is provided in the appendix.

*** Medicaid and non-Medicaid status are as of January 2015 for each individual. Individuals who carry both Medicare and Medicaid insurance ("dual eligibles") are classified as non-Medicaid enrollees. 
Table 2: Associations between Selected Characteristics and Time to Second Overdose Nonfatal Opioid Overdose Sample

(1)

(2)

(3)

(4)

Repeated OD Repeated OD Repeated OD Repeated OD

Number of Past 3 Months with MAT

0.962

$0.846^{* * *}$

$0.839^{* * *}$

(0.038)

(0.036)

$(0.037)$

$(0.051)$

Number of Past 3 Months with Behavioral Therapy

0.986

(0.056)

0.974

1.119

(0.058)

(0.213)

0.995

(0.160)

Receives MAT (Ever)

Behavioral Therapy (Ever)

Fills High-dose Opioid Rx (Ever)

Fills Both Opioid and Benzodiazepine Rx Same Month (Ever)

Opioid Use Disorder Diagnosis (Ever)

Alcohol Use Disorder Diagnosis (Ever)

Anxiety Diagnosis (Ever)

Depression Diagnosis (Ever)

Other Mental Illness Diagnosis (Ever)

Other Substance Abuse Disorder Diagnosis (Ever)

Hepatitis C Diagnosis (Ever)

Medicaid

First Overdose Age 31-42

First Overdose Age 43-52

First Overdose Age 53-90

Enters Sample 2012 (vs. 2011)

Number of Next 3 Months with MAT

Number Next 3 Months with Behavioral Therapy

\begin{tabular}{|c|c|c|c|c|}
\hline & & & & $(0.075)$ \\
\hline Fiscal Year Indicators & Yes & Yes & Yes & Yes \\
\hline Total Individuals in Sample & 2079 & 2079 & 2079 & 2079 \\
\hline Individuals with Two or More Overdoses & 539 & 539 & 539 & 539 \\
\hline Observations (Person-by-Month) & 70886 & 70886 & 70886 & 70886 \\
\hline
\end{tabular}

Source: Authors' calculations using HealthFacts RI.

Notes: The term "overdose" always refers to one that involved opioids. The dependent variable "repeated OD" is an indicator of having a second opioid overdose a given number of months after an initial (nonfatal) opioid overdose. All coefficients represent hazard ratios using Cox proportional hazard models. Standard errors are given in parentheses. A hazard ratio that is greater than 1.0 means that the factor is associated with an increased risk of having a repeated overdose relative to the baseline risk, as of any given number of months after the first overdose. The baseline hazard function was estimated separately (stratified) by gender. The estimation employs the exact partial likelihood method for treating tied second-overdose times across individuals. The sample is as described in the Notes to Table 1. All diagnosis-related variables, such as "alcohol use disorder (ever)," are defined as having at least one observation (either before or after the first overdose) with the given diagnosis. "Opioid use disorder" is defined as having at least one diagnosis corresponding to "opioid use," "opioid abuse," or "opioid dependence." The omitted category for first overdose age is ages 18 through 30. 
Figure 6: Cumulative Hazards (Probabilities) of Second Overdose for Women, by Selected Characteristics Nonfatal Opioid Overdose Sample

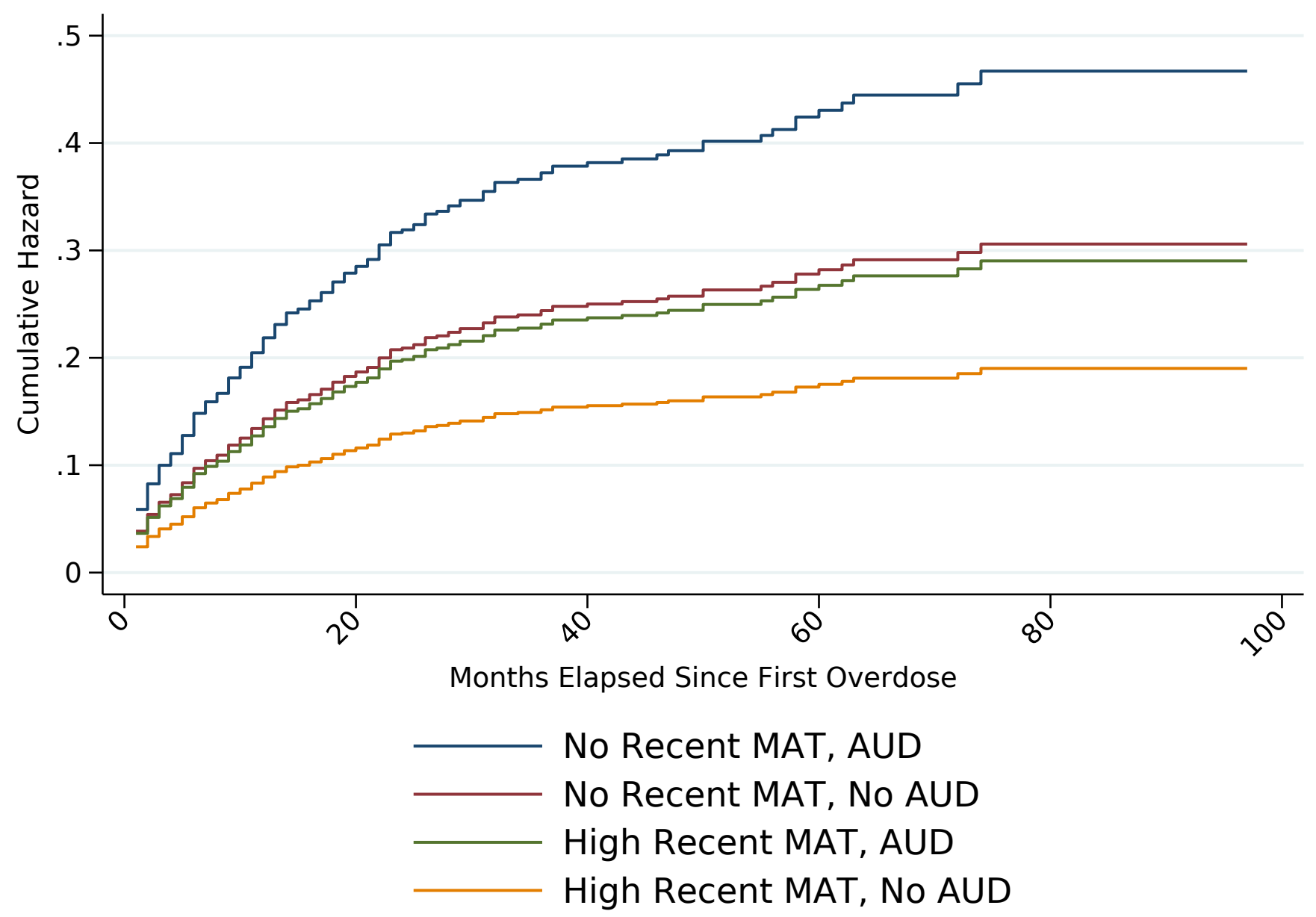

Source: Authors' calculations using HealthFacts RI.

Notes: The term "overdose" always refers to one that involved opioids. See Table 1 Notes for a description of the nonfatal opioid overdose sample. The cumulative hazard at a given point on a given line represents the estimated probability that an individual with the given characteristics will have had a second overdose within the given amount of elapsed time after the first overdose, based on a Cox proportional hazard model. "No recent MAT" means the individual did not receive either methadone or buprenorphine in any of the preceding three months, as of any amount of elapsed time. "High recent MAT" means the individual received either methadone or buprenorphine in all three of the preceding months, as of any amount of elapsed time. "AUD" means the individual received a diagnosis of alcohol use disorder at least once among all their observations, and "no AUD" means the individual never had a diagnosis of alcohol use disorder. Aside from the characteristics that vary in the figure, characteristics were held constant at the following values: female, diagnosed with opioid use disorder (ever), received either methadone or buprenorphine (ever), age at first overdose 18 through 30, and received no other diagnoses. All other variables were held at their baseline values, which are described in Section IV. 
Figure 7: Cumulative Hazards (Probabilities) of Second Overdose for Men, by Selected Characteristics Nonfatal Opioid Overdose Sample

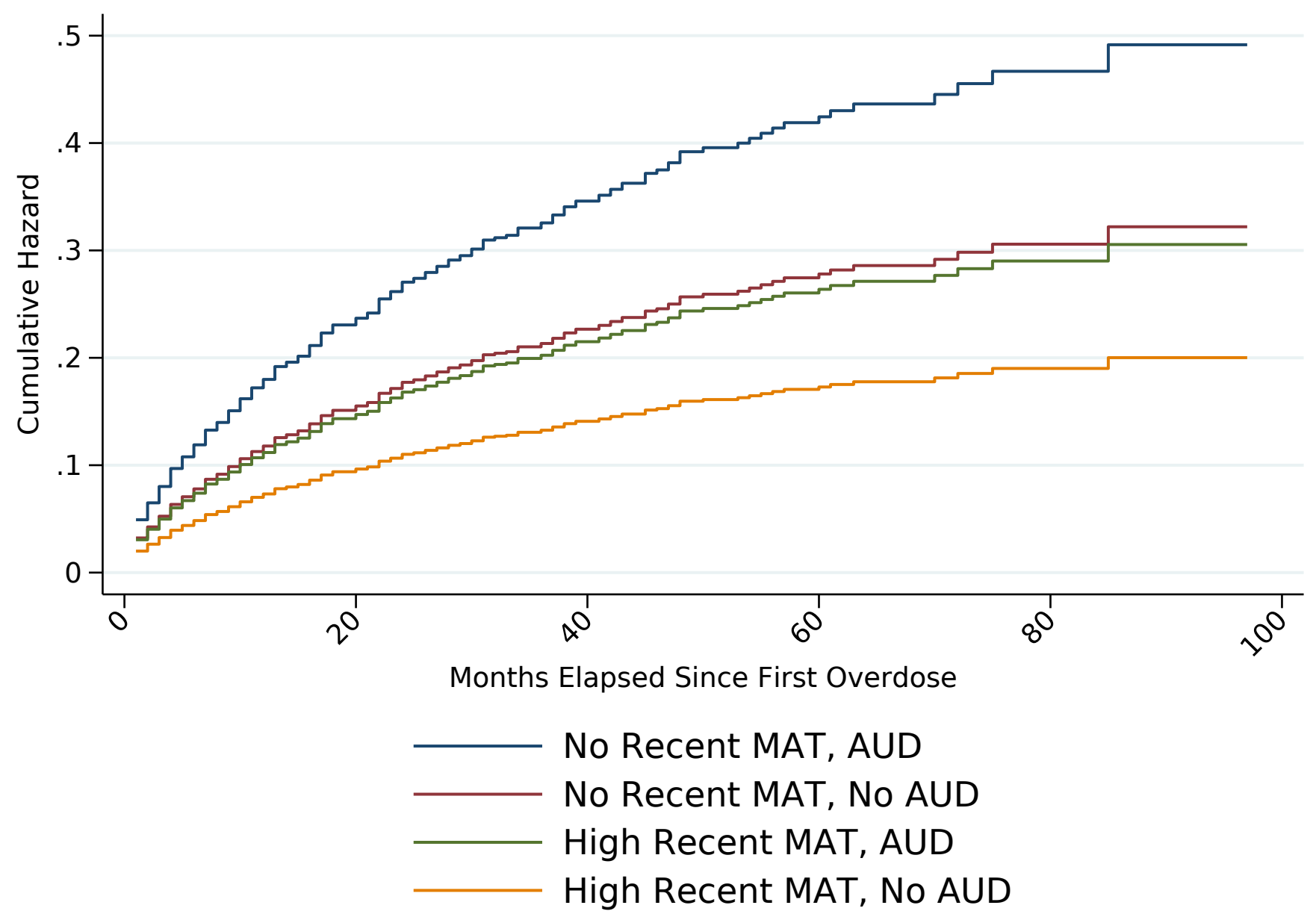

Source: Authors' calculations using HealthFacts RI.

Notes: The term "overdose" always refers to one that involved opioids. See Table 1 Notes for a description of the nonfatal opioid overdose sample. The cumulative hazard at a given point on a given line represents the estimated probability that an individual with the given characteristics will have had a second overdose within the given amount of elapsed time after the first overdose, based on a Cox proportional hazard model. "No recent MAT" means the individual did not receive either methadone or buprenorphine in any of the preceding three months, as of any amount of elapsed time. "High recent MAT" means the individual received either methadone or buprenorphine in all three of the preceding months, as of any amount of elapsed time. "AUD" means the individual received a diagnosis of alcohol use disorder at least once among all their observations, and "no AUD" means the individual never had a diagnosis of alcohol use disorder. Aside from the characteristics that vary in the figure, characteristics were held constant at the following values: male, diagnosed with opioid use disorder (ever), received either methadone or buprenorphine (ever), age at first overdose 18 through 30, and received no other diagnoses. All other variables were held at their baseline values, which are described in Section IV. 
Table 3: Percentage of Sample with Selected Characteristics Restricted Incumbent Panel and Subset with Opioid Dependence

\begin{tabular}{|c|c|c|}
\hline & All & Opioid Dependence (Ever) \\
\hline Received Buprenorphine* & 0.9 & 29.3 \\
\hline Received Methadone* & 0.6 & 19.7 \\
\hline Received MAT* & 1.3 & 42.7 \\
\hline Enters Sample 2011 & 92.5 & 90.5 \\
\hline Enters Sample 2012 & 7.5 & 9.5 \\
\hline Initial Age 18-44 & 34.6 & 52.8 \\
\hline Initial Age 45-64 & 36.8 & 40.8 \\
\hline Initial Age 65+ & 28.6 & 6.4 \\
\hline Medicaid & 7.6 & 31.5 \\
\hline Non-Medicaid & 92.4 & 68.5 \\
\hline Female & 55.2 & 46.9 \\
\hline Male & 44.8 & 53.1 \\
\hline Alcohol Use Disorder (Ever) & 7.3 & 41.1 \\
\hline Opioid Overdose (Ever) & 0.5 & 10.4 \\
\hline Hepatitis C (Ever) & 1.6 & 18.4 \\
\hline Depression (Ever) & 33.8 & 78.8 \\
\hline Anxiety (Ever) & 41.7 & 81 \\
\hline Other Mental Illness (Ever) & 42.1 & 85.1 \\
\hline Opioid Dependence (Ever) & 3.1 & 100 \\
\hline Other Substance Use Disorder (Ever) & 14.4 & 75.6 \\
\hline High Poverty Zip Code (Most Months) & 13.7 & 22.2 \\
\hline Median of Average Buprenorphine Spell Length & . & 15 \\
\hline Median of Average Methadone Spell Length & . & 26 \\
\hline Median of Average MAT Spell Length & . & 25.7 \\
\hline Sample Size & 288331 & 8951 \\
\hline
\end{tabular}

Source: Authors' calculations using HealthFacts RI.

Notes: The restricted incumbent panel consists of individuals who were observed, at a minimum, in most months (missing as many as three) from January 2013 through December 2015, who were at least age 19 as of January 2013, and who did not switch between Medicaid and non-Medicaid insurance plans between entry into the APCD and December 2016. The panel further excludes observations with United Healthcare insurance, observations with an unknown medical insurance carrier, out-of-state observations, observations with age under 18 , and individuals with missing poverty indicators in more than half of their observations. The opioid dependence (ever) sample consists of individuals in the restricted incumbent panel who ever received an opioid dependence diagnosis. Characteristics followed by an asterisk $\left({ }^{*}\right)$ are defined as having received buprenorphine/methadone/any MAT, respectively, in three or more months. Initial age is an individual's age as of their first observation in the given sample. Medicaid and non-Medicaid status are as of the earliest observation in 2013 for each individual. All diagnosis-related variables, such as "alcohol use disorder (ever)," are defined as having at least one observation with the given diagnosis. "High poverty Zip code (most months)" means that, for more than half of the months in which someone was observed, they resided in a Zip code in which 20 percent or more of households had incomes at or below the federal poverty level. In calculating median spell lengths for buprenorphine, methadone, and MAT, respectively, we drop all individuals with an average spell length of zero for the given medication. 
Table 4: Probit Models of the Probability of Having at Least Three Months of Buprenorphine/Methadone Treatment Opioid Dependence Sample within the Restricted Incumbent Panel

\begin{tabular}{|c|c|c|}
\hline & $\begin{array}{c}(1) \\
\text { Methadone (At Least } 3 \text { Months) }\end{array}$ & $\begin{array}{c}(2) \\
\text { Buprenorphine (At Least } 3 \text { Months) }\end{array}$ \\
\hline Initial Age 45-64 & $\begin{array}{c}-0.329^{* * *} \\
(0.037)\end{array}$ & $\begin{array}{c}-0.367^{* * *} \\
(0.032)\end{array}$ \\
\hline Initial Age 65+ & $\begin{array}{c}-0.851^{* * *} \\
\quad(0.115)\end{array}$ & $\begin{array}{c}-1.019^{* * *} \\
(0.086)\end{array}$ \\
\hline Medicaid & $\begin{array}{l}0.701^{* * *} \\
(0.037)\end{array}$ & $\begin{array}{c}0.033 \\
(0.033)\end{array}$ \\
\hline Female & $\begin{array}{c}-0.079^{* *} \\
(0.036)\end{array}$ & $\begin{array}{l}-0.106^{* * *} \\
(0.030)\end{array}$ \\
\hline Alcohol Use Disorder (Ever) & $\begin{array}{c}-0.379^{* * *} \\
(0.037)\end{array}$ & $\begin{array}{l}-0.004 \\
(0.031)\end{array}$ \\
\hline Opioid Overdose (Ever) & $\begin{array}{c}0.321^{* * *} \\
(0.052)\end{array}$ & $\begin{array}{c}0.249^{* * *} \\
(0.046)\end{array}$ \\
\hline Hepatitis C (Ever) & $\begin{array}{l}0.963^{* * *} \\
(0.041)\end{array}$ & $\begin{array}{c}0.214^{* * *} \\
(0.038)\end{array}$ \\
\hline Depression (Ever) & $\begin{array}{c}0.014 \\
(0.050)\end{array}$ & $\begin{array}{l}-0.010 \\
(0.042)\end{array}$ \\
\hline Anxiety (Ever) & $\begin{array}{l}-0.067 \\
(0.049)\end{array}$ & $\begin{array}{c}0.054 \\
(0.043)\end{array}$ \\
\hline Other Mental Illness (Ever) & $\begin{array}{c}0.035 \\
(0.056)\end{array}$ & $\begin{array}{l}-0.008 \\
(0.048)\end{array}$ \\
\hline Other Substance Use Disorder (Ever) & $\begin{array}{l}0.239^{* * *} \\
(0.046)\end{array}$ & $\begin{array}{c}0.510^{* * *} \\
(0.040)\end{array}$ \\
\hline High-poverty Zip Code & $\begin{array}{c}0.110^{* * *} \\
(0.040)\end{array}$ & $\begin{array}{c}-0.182^{* * *} \\
(0.036)\end{array}$ \\
\hline Constant & $\begin{array}{c}-1.267^{* * *} \\
\quad(0.061)\end{array}$ & $\begin{array}{c}-0.780^{* * *} \\
(0.052)\end{array}$ \\
\hline Observations & 8951 & 8951 \\
\hline
\end{tabular}

Source: Authors' calculations using HealthFacts RI.

Notes: Robust standard errors in parentheses. See Notes to Table 3 for a description of the sample. Initial age is an individual's age as of their first observation in the analysis sample. Medicaid status is as of the earliest observation in 2013 for each individual. All diagnosis-related variables, such as "Alcohol use disorder (ever)," are defined as having at least one observation with the given diagnosis. "High-poverty Zip code" means that, as of January 2015, the individual resided in a Zip code in which 20 percent or more of households had incomes at or below the federal poverty level. 
Figure 8: Predicted Probability of Receiving Methadone Treatment

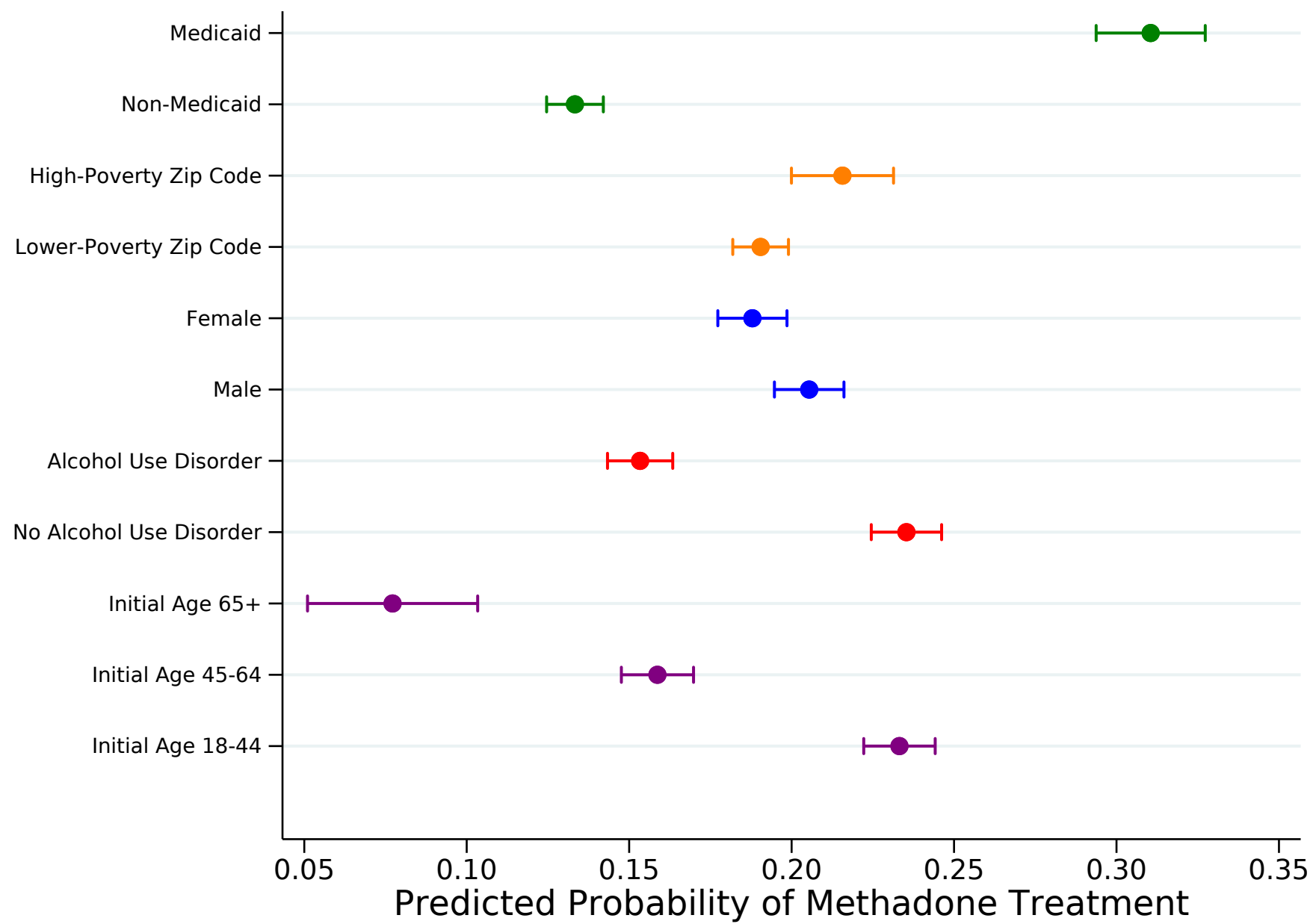

Source: Authors' calculations using HealthFacts RI.

Notes: Probabilities are based on a multivariate probit model of receiving methadone treatment in at least three separate months. Sample consists of patients in the restricted incumbent panel who were diagnosed with opioid dependence. "High-poverty Zip code" means that, for more than half of the months in which someone was observed, they resided in a Zip code in which 20 percent or more of households had incomes at or below the federal poverty level. "Lower-poverty Zip code" means that the individual did not meet the criterion for "high-poverty Zip code." "Alcohol use disorder" means the individual received a diagnosis of alcohol use disorder at least once among all their observations, and "no alcohol use disorder" means the individual never had a diagnosis of alcohol use disorder. Initial age is an individual's age as of their first observation in the analysis sample. 
Figure 9: Predicted Probability of Receiving Buprenorphine Prescriptions

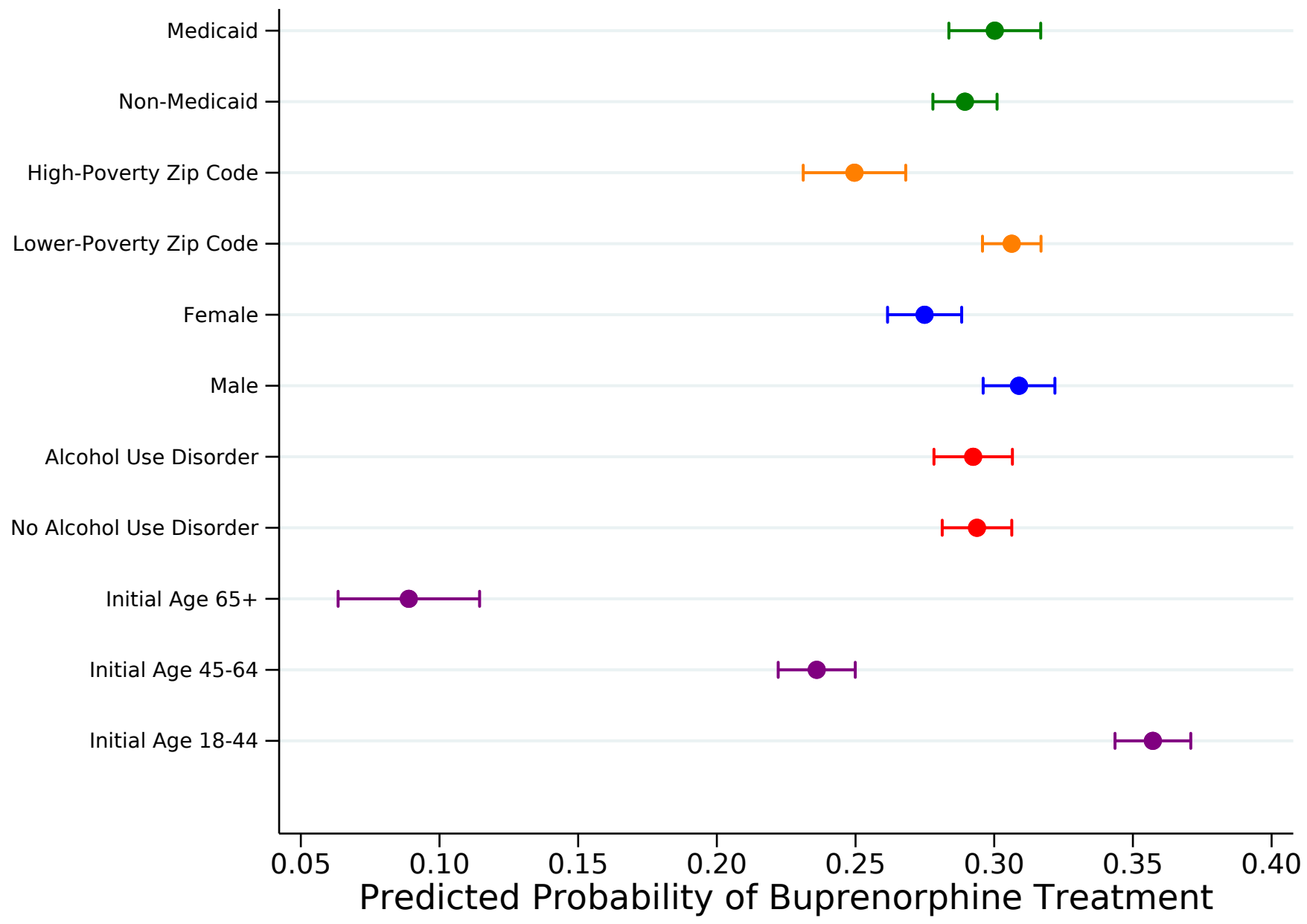

Source: Authors' calculations using HealthFacts RI.

Notes: Probabilities are based on a multivariate probit model of receiving a buprenorphine prescription in at least three separate months. Sample consists of patients in the restricted incumbent panel who were diagnosed with opioid dependence. "High-poverty Zip code" means that, for more than half of the months in which someone was observed, they resided in a Zip code in which 20 percent or more of households had incomes at or below the federal poverty level. "Lower-poverty Zip code" means that the individual did not meet the criterion for "high-poverty Zip code." "Alcohol use disorder" means the individual received a diagnosis of alcohol use disorder at least once among all their observations, and "no alcohol use disorder" means the individual never had a diagnosis of alcohol use disorder. Initial age is an individual's age as of their first observation in the analysis sample. 
Figure 10: Six-month Average Buprenorphine Patient Loads, September 2015-August 2017

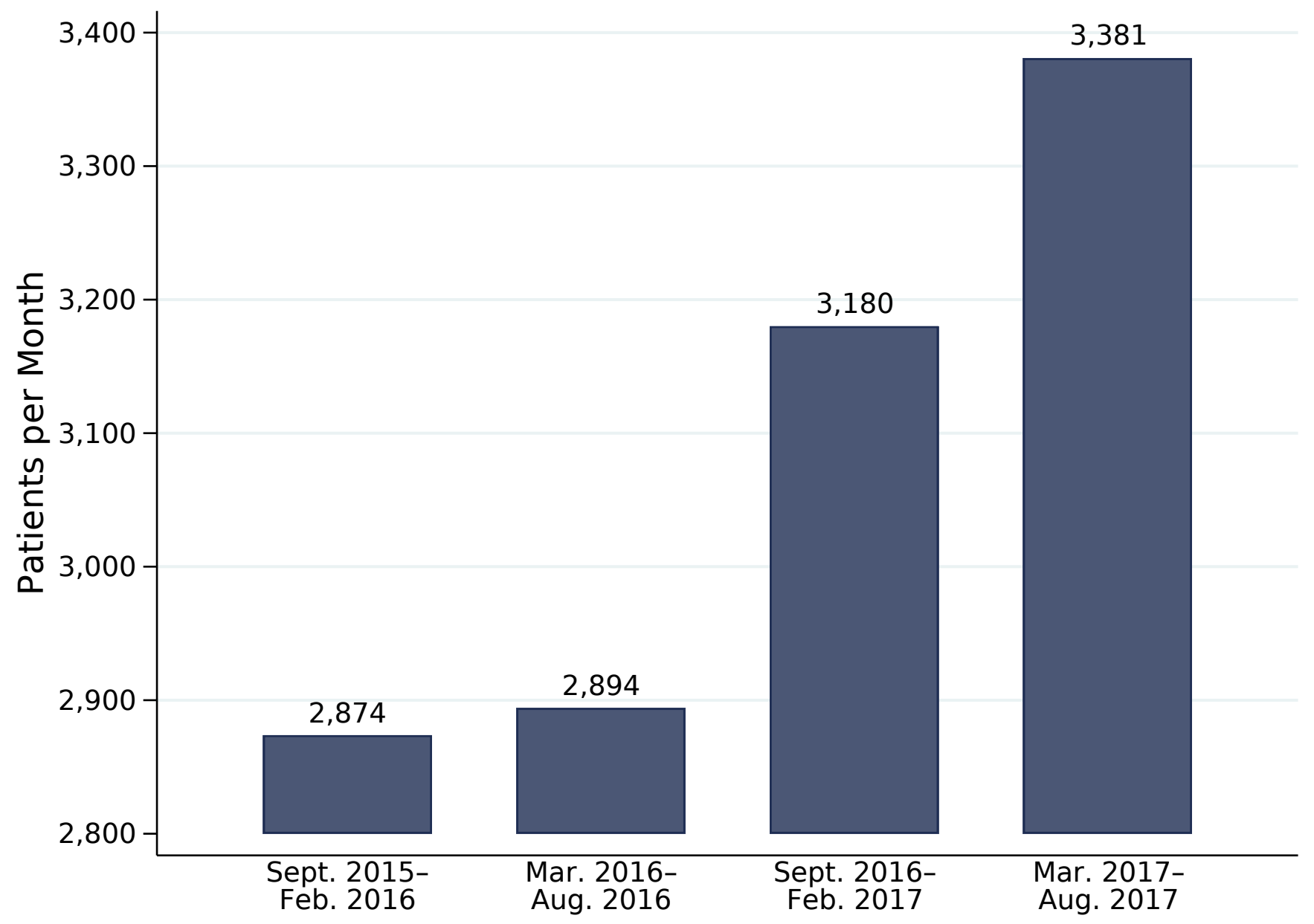

Source: Authors' calculations using HealthFacts RI.

Notes: The patient load in a given month is the number of unique enrollees (ages 18 and older and residing in Rhode Island) who filled an allowable buprenorphine prescription in that month. A patient is counted at most once per month regardless of how many prescriptions they filled. An allowable prescription involves a buprenorphine formulation that is FDA-approved to treat OUD and for which the insurance claim was not denied. The prescriber on the claim must be an individual (not an organization) with a Rhode Island Zip code. The activity of one prescriber with excessive patient loads was excluded. 
Figure 11: Buprenorphine Prescriber Stock and Average Number of Buprenorphine Patients per Prescriber Before and After September 2016

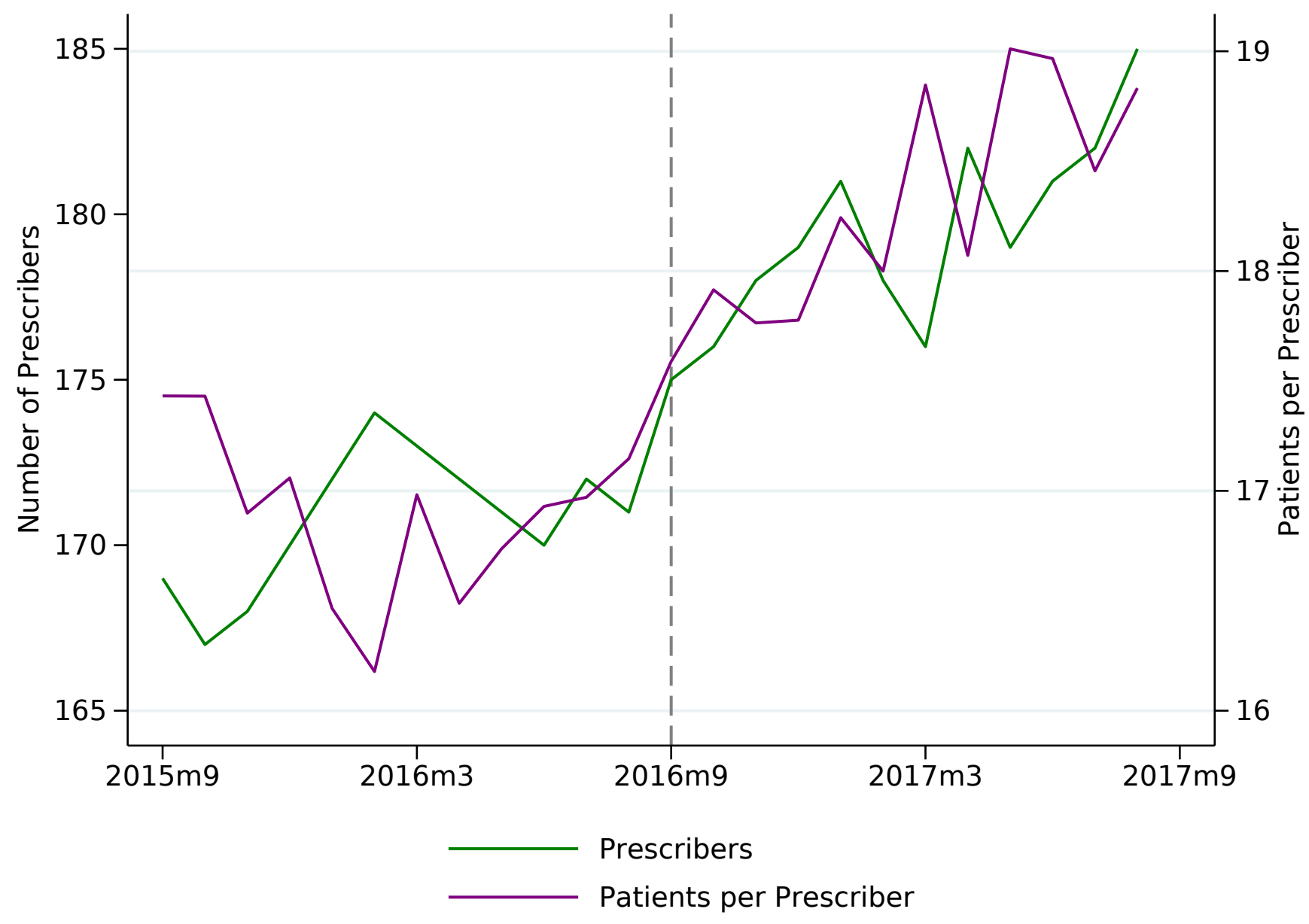

Source: Authors' calculations using HealthFacts RI.

Notes: The vertical dashed line at September 2016 demarks the first month following changes in federal buprenorphine prescribing policies. The monthly prescriber stock includes only active prescribers: The prescriber must appear on a buprenorphine claim dated in that month or on claims dated both before and after the given month. Patients per prescriber is the ratio of the patient load to the prescriber stock. See Figure 10 Notes for the methods of counting the patient load per month. 
Figure 12: True Buprenorphine Patient Load and Counterfactual Buprenorphine Patient Loads Before and After September 2016

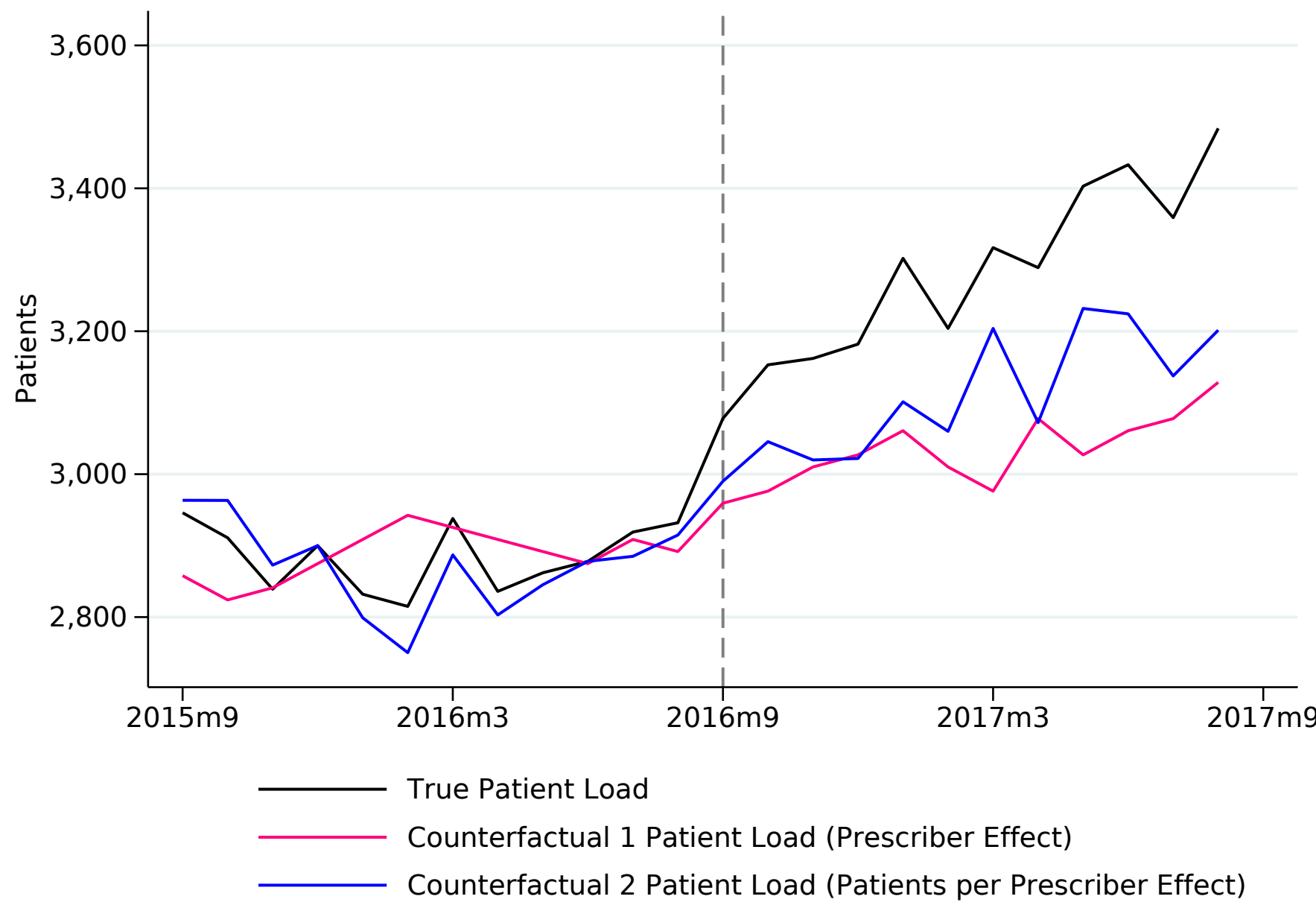

Source: Authors' calculations using HealthFacts RI.

Notes: The vertical dashed line at September 2016 demarks the first month following changes in federal buprenorphine prescribing policies. See Figure 10 Notes for the method of calculating the patient load and Figure 11 Notes for the methods of calculating the prescriber stock and patients per prescriber. To calculate counterfactual 1, the value of patients per prescriber in a given month was multiplied by the fixed baseline value of the prescriber stock. The latter was set at 170, the average for September 2015 through February 2016. To calculate counterfactual 2, the prescriber stock in a given month was multiplied by the fixed baseline value of patients per prescriber. The latter was set at 16.9, the average for September 2015 through February 2016. 
Figure 13: Percentiles of the Patients-per-Prescriber Distribution, Before and After September 2016

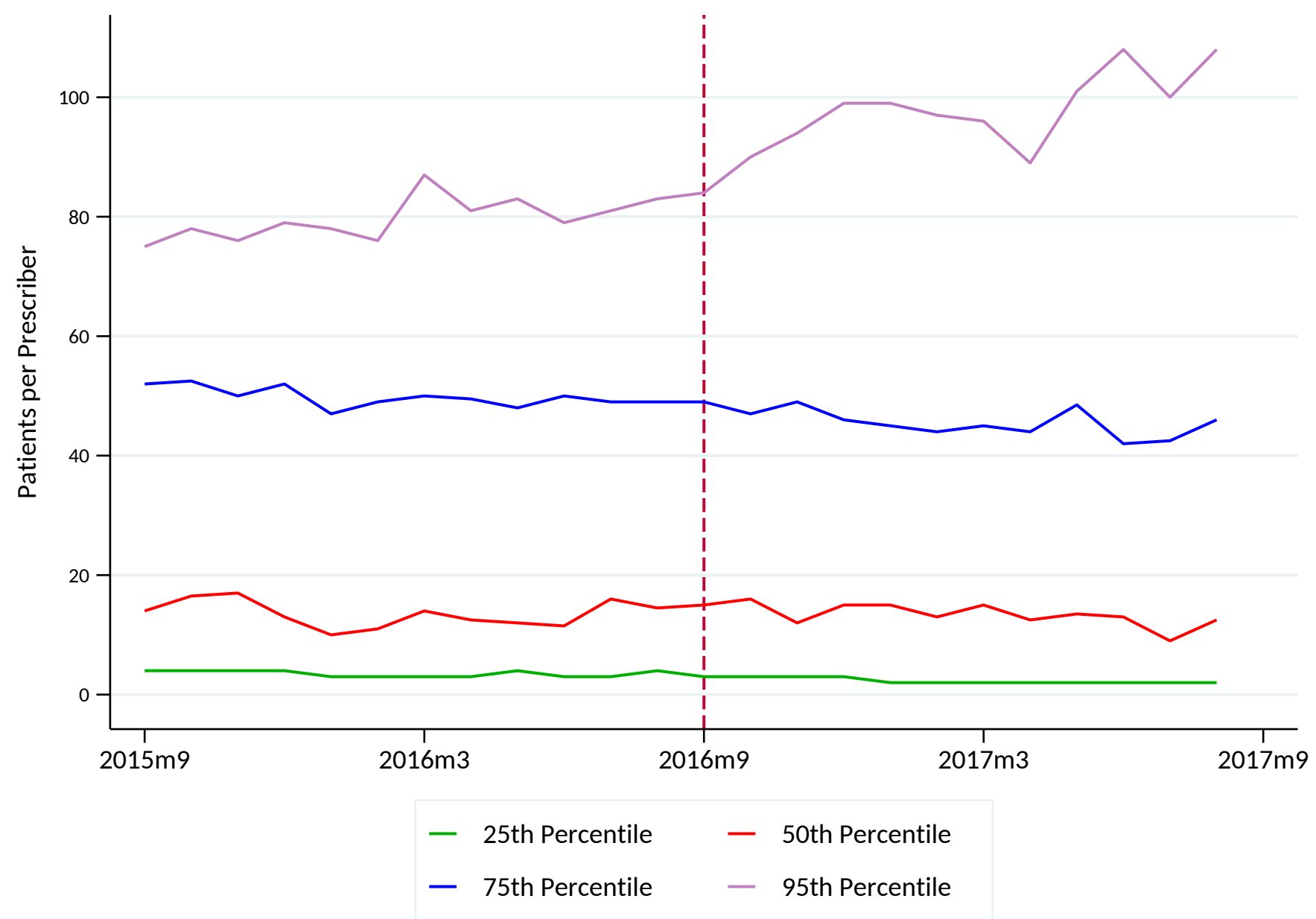

Source: Authors' calculations using HealthFacts RI.

Notes: The vertical dashed line at September 2016 demarks the first month following changes in federal buprenorphine prescribing policies. See Figure 11 Notes for the method of calculating the patients per prescriber in a given month. In calculating the monthly percentiles, we include only Rhode Island-based prescribers that wrote at least one prescription for buprenorphine in the given month. We exclude organizations and one prescriber with excessive patient loads. 
Figure 14: Composition of Credentials among Buprenorphine Prescribers Before and After September 2016 By Patient's Initial Treatment Date

Prescribers to Incumbent Patients

September 2015-August 2016

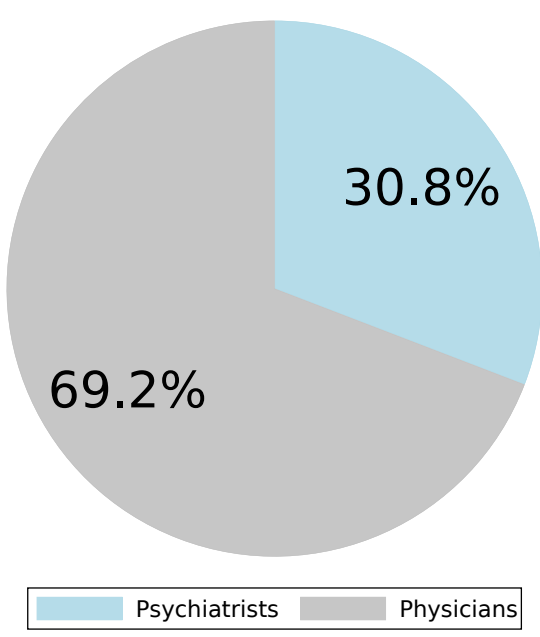

Prescribers to Incumbent Patients

September 2016-August 2017

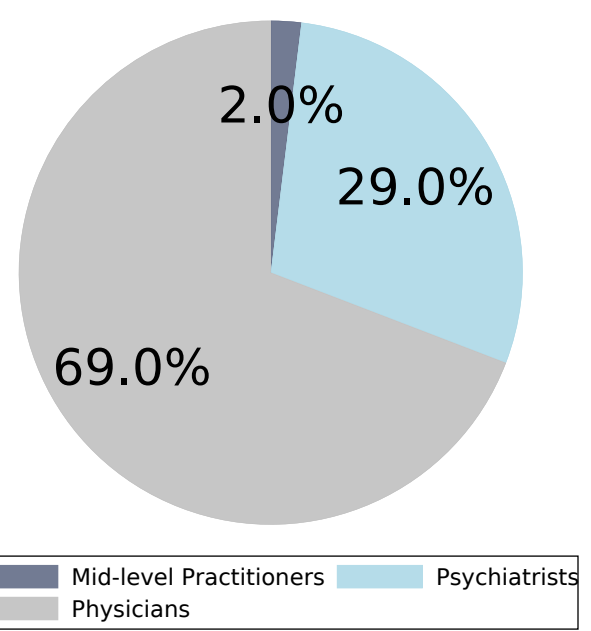

Prescribers to Newly Treated Patients

September 2016-August 2017

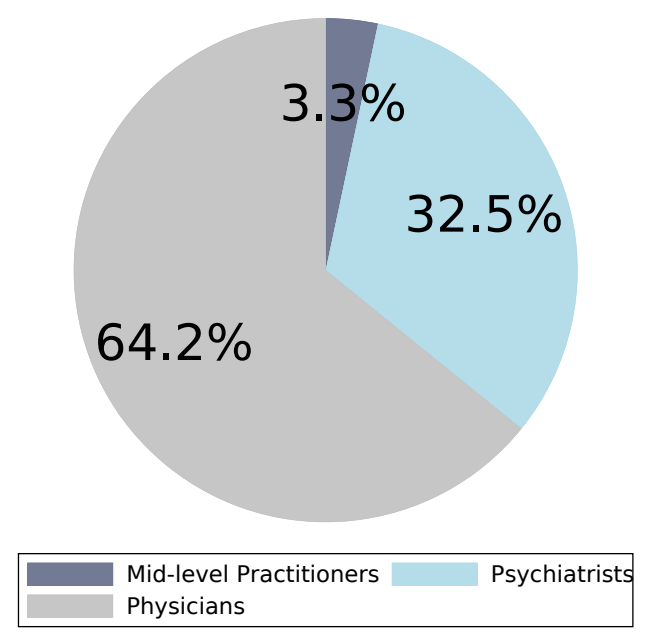

Source: Authors' calculations using HealthFacts RI.

Notes: September 2016 was the first month following changes in federal buprenorphine prescribing policies. In a given pie, the universe of observations is the set of unique patient-provider pairs associated with the buprenorphine prescriptions observed in the given time period for patients with the relevant characteristic. A given slice represents the share of observations in which the provider has the relevant credential. Incumbent patients are those first observed with a buprenorphine prescription prior to September 2016, and newly treated patients are those who first receive buprenorphine in September 2016 or later. The set of prescribers excludes organizations and one prescriber with excessive patients loads. The category of physicians includes nonpsychiatrists with either an MD degree (medical doctor) or DO degree (doctor of osteopathic medicine). Mid-level practitioners include physician assistants and nurse practitioners. 
Figure 15: Composition of Credentials among Buprenorphine Prescribers Before and After September 2016 By Patient's Zip Code Poverty Status

Prescribers to Patients in Lower-Poverty Zip Codes September 2015-August 2016

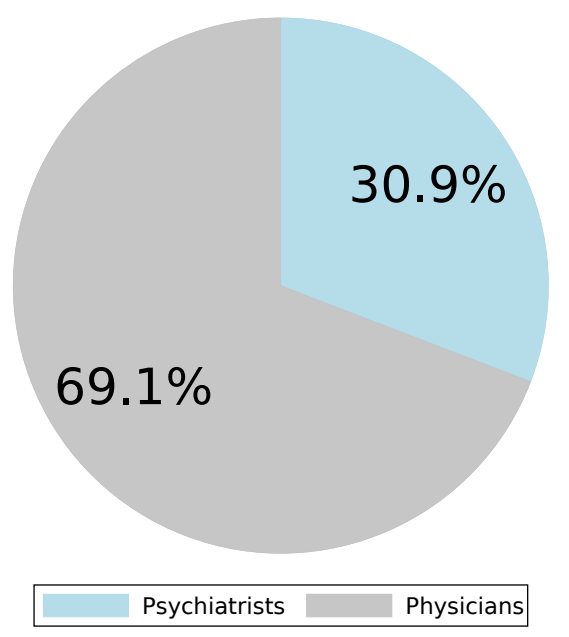

Prescribers to Patients in High-Poverty Zip Codes September 2015-August 2016

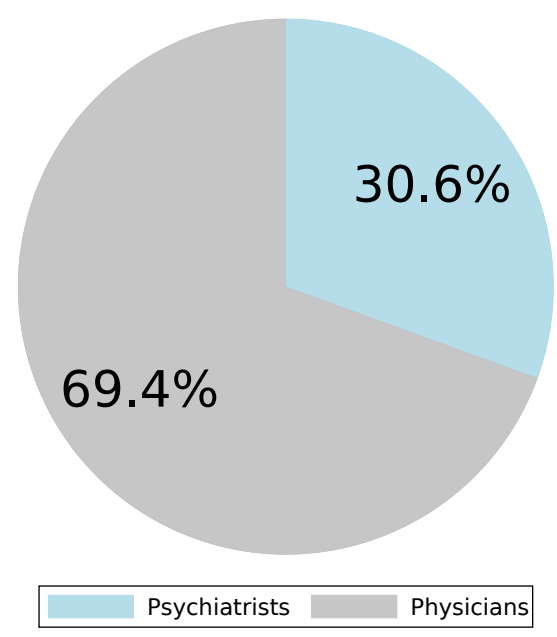

Prescribers to Patients in Lower-Poverty Zip Codes September 2016-August 2017

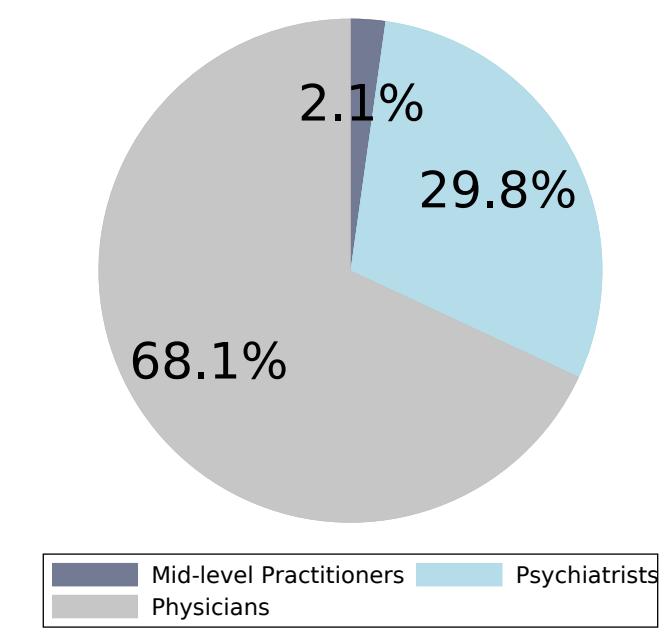

Prescribers to Patients in High-Poverty Zip Codes September 2016-August 2017

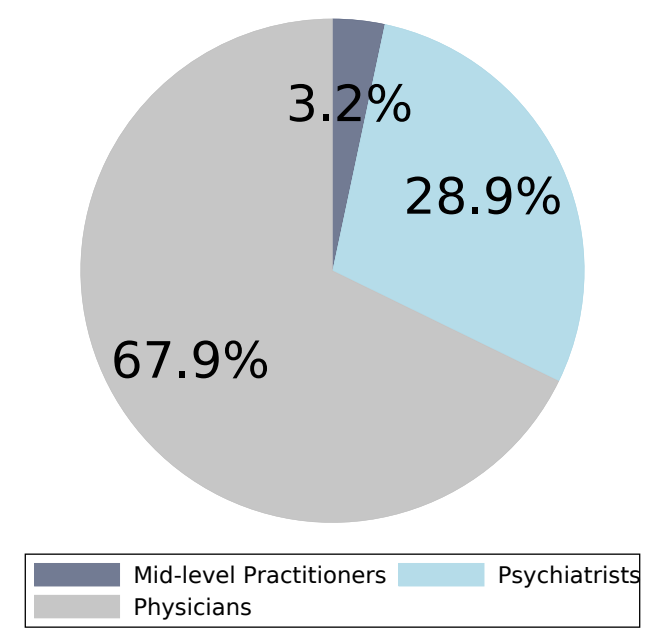

Sources: Authors' calculations using HealthFacts RI and the American Community Survey.

Notes: September 2016 was the first month following changes in federal buprenorphine prescribing policies. In a given pie, the universe of observations is the set of unique patient-provider pairs associated with the buprenorphine prescriptions observed in the given time period for patients with the relevant characteristic. A given slice represents the share of observations in which the provider has the relevant credential. Lower-poverty Zip codes are those in which less than 20 percent of households had incomes at or below the federal poverty level in the given year, and high-poverty Zip codes are those in which 20 percent or more of households had poverty-level or lower incomes in the given year. The set of prescribers excludes organizations and one prescriber with excessive patient loads. The category of physicians includes non-psychiatrists with either an MD degree (medical doctor) or DO degree (doctor of osteopathic medicine). Mid-level practitioners include physician assistants and nurse practitioners. 
Figure 16: Annual Buprenorphine Prescribers: HealthFacts RI vs. All RI Prescribers

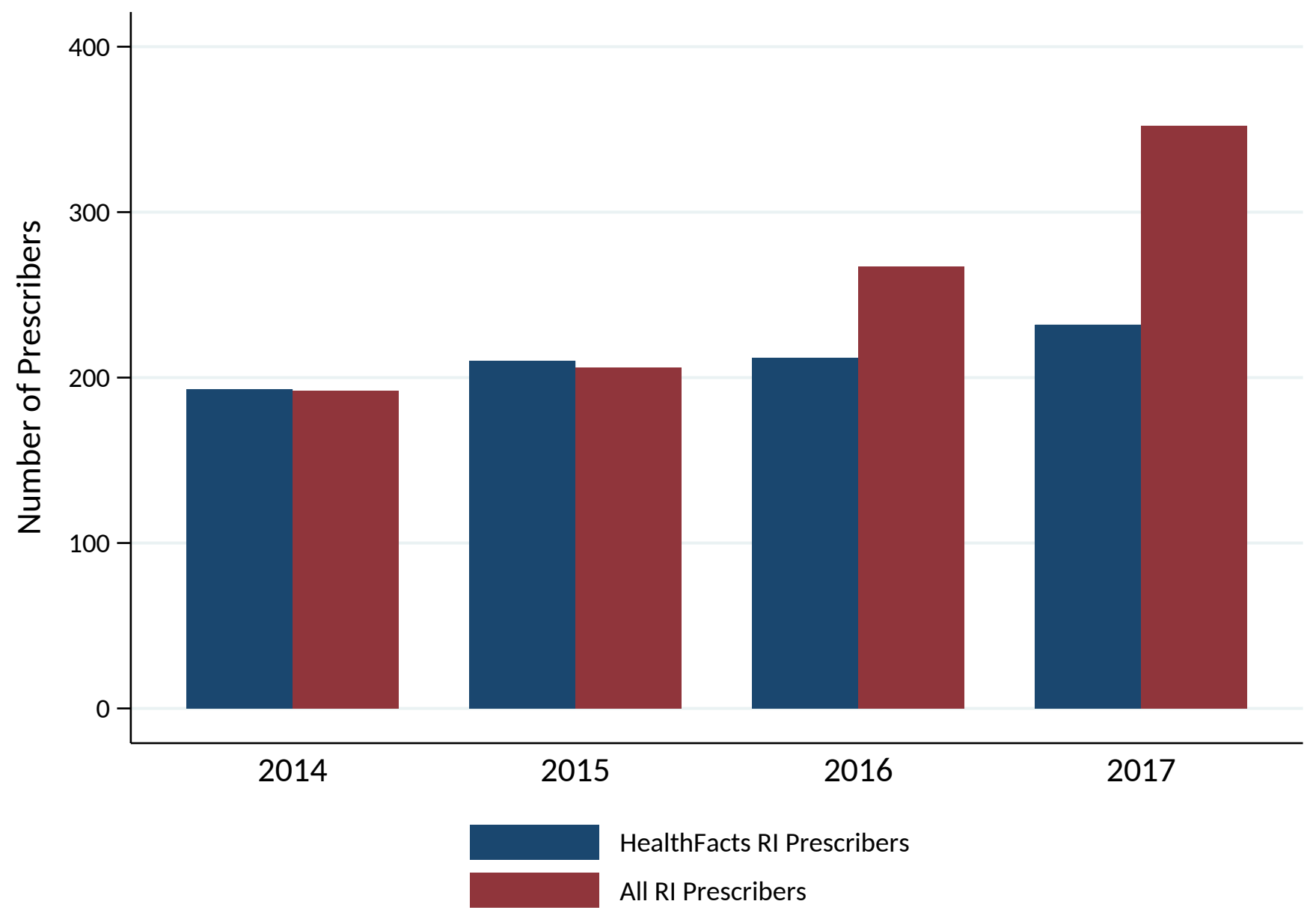

Sources: Authors' calculations using HealthFacts RI and Rhode Island Department of Health.

Notes: "HealthFacts RI prescribers" in a given year refers to the number of Rhode Island-based prescribers associated with at least one buprenorphine prescription observed in the database in that year, excluding organizations and one prescriber with excessive patient loads. "All RI prescribers" refers to the total number of waivered Rhode Island prescribers in the state in a given year. 
Appendix for "Who Gets Medication-assisted Treatment for Opioid Use Disorder and Does It Reduce Overdose Risk? Evidence from the Rhode Island All-payer Claims Database."

Mary A. Burke, Riley Sullivan, Katherine Carman, Hefei Wen, J. Frank Wharam, and Hao Yu 
Table A1: Associations between Selected Characteristics and Time to Second Overdose Robustness Tests

\begin{tabular}{|c|c|c|c|c|}
\hline & $\begin{array}{c}(1) \\
\text { Repeated OD }\end{array}$ & $\begin{array}{c}(2) \\
\text { Repeated OD }\end{array}$ & $\begin{array}{c}(3) \\
\text { Repeated OD }\end{array}$ & $\begin{array}{c}(4) \\
\text { Repeated OD }\end{array}$ \\
\hline Number of Past 3 Months with MAT & $\begin{array}{c}0.857^{* * *} \\
(0.036)\end{array}$ & $\begin{array}{c}0.850^{* * * *} \\
(0.037)\end{array}$ & $\begin{array}{c}0.807^{* * *} \\
(0.045)\end{array}$ & $\begin{array}{c}0.843^{* * *} \\
(0.036)\end{array}$ \\
\hline Number of Past 3 Months with Behavioral Therapy & $\begin{array}{c}0.971 \\
(0.056)\end{array}$ & $\begin{array}{c}0.982 \\
(0.059)\end{array}$ & $\begin{array}{c}0.994 \\
(0.068)\end{array}$ & $\begin{array}{c}0.904 \\
(0.069)\end{array}$ \\
\hline Fills High-dose Opioid Rx in Current Month & $\begin{array}{c}1.383 \\
(0.278)\end{array}$ & $\begin{array}{c}1.394 \\
(0.283)\end{array}$ & $\begin{array}{c}1.152 \\
(0.335)\end{array}$ & $\begin{array}{c}1.119 \\
(0.384)\end{array}$ \\
\hline Fills Both Opioid and Benzodiazepine Rx in Current Month & $\begin{array}{c}1.221 \\
(0.206)\end{array}$ & $\begin{array}{c}1.224 \\
(0.208)\end{array}$ & $\begin{array}{c}1.329 \\
(0.291)\end{array}$ & $\begin{array}{c}1.257 \\
(0.314)\end{array}$ \\
\hline Receives MAT (Ever) & $\begin{array}{c}1.813^{* * *} \\
(0.206)\end{array}$ & $\begin{array}{c}1.828^{* * *} \\
(0.219)\end{array}$ & $\begin{array}{c}2.041^{* * *} \\
(0.315)\end{array}$ & \\
\hline Behavioral Therapy (Ever) & $\begin{array}{c}1.027 \\
(0.111)\end{array}$ & $\begin{array}{c}1.014 \\
(0.116)\end{array}$ & $\begin{array}{c}1.137 \\
(0.206)\end{array}$ & $\begin{array}{c}0.973 \\
(0.140)\end{array}$ \\
\hline Fills High-dose Opioid Rx (Ever) & $\begin{array}{c}1.059 \\
(0.110)\end{array}$ & $\begin{array}{c}1.066 \\
(0.114)\end{array}$ & $\begin{array}{c}1.115 \\
(0.168)\end{array}$ & $\begin{array}{c}0.860 \\
(0.114)\end{array}$ \\
\hline Fills Both Opioid and Benzodiazepine Rx Same Month (Ever) & $\begin{array}{c}0.968 \\
(0.098)\end{array}$ & $\begin{array}{c}0.958 \\
(0.100)\end{array}$ & $\begin{array}{c}0.952 \\
(0.127)\end{array}$ & $\begin{array}{c}1.036 \\
(0.128)\end{array}$ \\
\hline Opioid Use Disorder Diagnosis (Ever) & $\begin{array}{c}1.616^{* * *} \\
(0.282)\end{array}$ & $\begin{array}{c}1.648^{* * *} \\
(0.292)\end{array}$ & $\begin{array}{l}1.470^{*} \\
(0.321)\end{array}$ & \\
\hline Alcohol Use Disorder Diagnosis (Ever) & $\begin{array}{c}1.513^{* * *} \\
(0.153)\end{array}$ & $\begin{array}{c}1.497^{* * *} \\
(0.159)\end{array}$ & $\begin{array}{c}1.867^{* * *} \\
(0.271)\end{array}$ & $\begin{array}{c}1.725^{* * *} \\
(0.237)\end{array}$ \\
\hline Anxiety Diagnosis (Ever) & $\begin{array}{c}1.213 \\
(0.245)\end{array}$ & $\begin{array}{c}1.384 \\
(0.295)\end{array}$ & $\begin{array}{c}1.273 \\
(0.337)\end{array}$ & $\begin{array}{c}1.012 \\
(0.286)\end{array}$ \\
\hline Depression Diagnosis (Ever) & $\begin{array}{c}0.889 \\
(0.167)\end{array}$ & $\begin{array}{c}0.908 \\
(0.175)\end{array}$ & $\begin{array}{c}0.672 \\
(0.164)\end{array}$ & $\begin{array}{c}1.519 \\
(0.474)\end{array}$ \\
\hline Other Mental Illness Diagnosis (Ever) & $\begin{array}{c}1.429 \\
(0.378)\end{array}$ & $\begin{array}{c}1.302 \\
(0.334)\end{array}$ & $\begin{array}{c}1.554 \\
(0.569)\end{array}$ & $\begin{array}{c}1.001 \\
(0.368)\end{array}$ \\
\hline Other Substance Abuse Disorder Diagnosis (Ever) & $\begin{array}{c}1.098 \\
(0.228)\end{array}$ & $\begin{array}{c}1.096 \\
(0.231)\end{array}$ & $\begin{array}{c}0.954 \\
(0.256)\end{array}$ & $\begin{array}{c}1.806 \\
(1.071)\end{array}$ \\
\hline Hepatitis C Diagnosis (Ever) & $\begin{array}{c}1.396^{* * *} \\
(0.128)\end{array}$ & $\begin{array}{c}1.416^{* * *} \\
(0.137)\end{array}$ & $\begin{array}{c}1.568^{* * *} \\
(0.196)\end{array}$ & $\begin{array}{c}1.606^{* * *} \\
(0.185)\end{array}$ \\
\hline Medicaid & $\begin{array}{c}0.995 \\
(0.098)\end{array}$ & $\begin{array}{c}1.014 \\
(0.104)\end{array}$ & $\begin{array}{c}1.025 \\
(0.140)\end{array}$ & $\begin{array}{c}0.853 \\
(0.104)\end{array}$ \\
\hline First Overdose Age 31-42 & $\begin{array}{l}0.744^{* *} \\
(0.091)\end{array}$ & $\begin{array}{c}0.765^{* *} \\
(0.100)\end{array}$ & $\begin{array}{l}0.733^{*} \\
(0.119)\end{array}$ & $\begin{array}{l}0.696^{* *} \\
(0.101)\end{array}$ \\
\hline First Overdose Age 43-52 & $\begin{array}{c}0.616^{* * *} \\
(0.080)\end{array}$ & $\begin{array}{c}0.613^{* * *} \\
(0.086)\end{array}$ & $\begin{array}{c}0.537^{* * *} \\
(0.098)\end{array}$ & $\begin{array}{c}0.553^{* * *} \\
(0.088)\end{array}$ \\
\hline First Overdose Age 53-90 & $\begin{array}{c}0.648^{* * *} \\
(0.088)\end{array}$ & $\begin{array}{c}0.657^{* * *} \\
(0.094)\end{array}$ & $\begin{array}{c}0.598^{* * *} \\
(0.111)\end{array}$ & $\begin{array}{c}0.536^{* * *} \\
(0.094)\end{array}$ \\
\hline Enters Sample 2012 (vs. 2011) & $\begin{array}{l}1.338^{* *} \\
(0.197)\end{array}$ & $\begin{array}{l}1.305^{*} \\
(0.195)\end{array}$ & $\begin{array}{l}1.412^{*} \\
(0.277)\end{array}$ & $\begin{array}{l}1.359^{*} \\
(0.230)\end{array}$ \\
\hline Fiscal Year Indicators & Yes & Yes & Yes & Yes \\
\hline Total Individuals in Sample & 2079 & 1981 & 1362 & 993 \\
\hline Individuals with Two or More Overdoses & 539 & 528 & 318 & 361 \\
\hline Observations (Person-by-Month) & 70886 & 70324 & 46785 & 30790 \\
\hline
\end{tabular}

Source: Authors' calculations using HealthFacts RI.

Notes: All coefficients represent hazard ratios using Cox proportional hazard models stratified by gender (see Notes to Table 2 for a discussion of the model and definitions of variables). Standard errors are given in parentheses. All columns display estimation results for the third model in Table 2 using the sample described in the Notes to Table 1, with the following exceptions: column 1 uses the Breslow method to handle tied second-overdose times across individuals and reports robust standard errors. Column 2 requires that a patient be observed in at least six consecutive months following their first overdose. Column 3 excludes observations with United Healthcare insurance. Column 4 is restricted to those individuals who received MAT at least once (all but two such individuals had an OUD diagnosis at least once, therefore the OUD covariate is omitted from the model). 
Table A2: Probit Models of the Probability of Receiving Methadone/Buprenorphine Treatment Robustness Tests

\begin{tabular}{lcc|cc}
\hline & \multicolumn{2}{c}{ Methadone } & \multicolumn{2}{c}{ Buprenorphine } \\
& $(1)$ & $(2)$ & $(3)$ & $(4)$ \\
& One Month & Three Months & One Month & Three Months \\
& (Incumbent Sample) & (Basic Sample) & (Incumbent Sample) & $($ Basic Sample) \\
\hline Initial Age 45-64 & $-0.323^{* * *}$ & $-0.315^{* * *}$ & $-0.389^{* * *}$ & $-0.326^{* * *}$ \\
& $(0.036)$ & $(0.023)$ & $(0.031)$ & $(0.020)$ \\
Initial Age 65+ & $-0.911^{* * *}$ & $-0.712^{* * *}$ & $-0.997^{* * *}$ & $-1.006^{* * *}$ \\
& $(0.115)$ & $(0.070)$ & $(0.081)$ & $(0.061)$ \\
Medicaid & $0.710^{* * *}$ & $0.610^{* * *}$ & $0.071^{* *}$ & $-0.043^{* *}$ \\
& $(0.036)$ & $(0.021)$ & $(0.033)$ & $(0.019)$ \\
Female & $-0.089^{* *}$ & $-0.073^{* * *}$ & $-0.115^{* * *}$ & $-0.075^{* * *}$ \\
& $(0.035)$ & $(0.021)$ & $(0.030)$ & $(0.019)$ \\
Alcohol Use Disorder (Ever) & $-0.345^{* * *}$ & $-0.392^{* * *}$ & 0.001 & $-0.069^{* * *}$ \\
& $(0.037)$ & $(0.022)$ & $(0.031)$ & $(0.019)$ \\
Opioid Overdose (Ever) & $0.396^{* * *}$ & $0.352^{* * *}$ & $0.335^{* * *}$ & $0.279^{* * *}$ \\
& $(0.051)$ & $(0.031)$ & $(0.046)$ & $(0.028)$ \\
Hepatitis C (Ever) & $0.939^{* * *}$ & $0.853^{* * *}$ & $0.217^{* * *}$ & $0.164^{* * *}$ \\
& $(0.041)$ & $(0.025)$ & $(0.038)$ & $(0.024)$ \\
Depression (Ever) & -0.022 & 0.041 & 0.026 & -0.011 \\
& $(0.048)$ & $(0.026)$ & $(0.041)$ & $(0.024)$ \\
Anxiety (Ever) & -0.077 & $-0.102^{* * *}$ & 0.044 & $0.107^{* * *}$ \\
Other Mental Illness (Ever) & $(0.048)$ & $(0.026)$ & $(0.042)$ & $(0.024)$ \\
Other Substance Use Disorder (Ever) & 0.031 & -0.001 & -0.023 & -0.018 \\
& $(0.054)$ & $(0.027)$ & $(0.047)$ & $(0.025)$ \\
High-poverty Zip Code & $0.238^{* * *}$ & $0.043^{*}$ & $0.523^{* * *}$ & $0.388^{* * *}$ \\
& $(0.045)$ & $(0.025)$ & $(0.039)$ & $(0.024)$ \\
Constant & $0.104^{* * *}$ & $0.045^{*}$ & $-0.136^{* * *}$ & $-0.205^{* * *}$ \\
& $(0.039)$ & $(0.024)$ & $(0.035)$ & $(0.022)$ \\
Observations & $-1.175^{* * *}$ & $-0.974^{* * *}$ & $-0.701^{* * *}$ & $-0.651^{* * *}$ \\
\hline & $(0.059)$ & $(0.030)$ & $(0.051)$ & $(0.027)$ \\
\hline
\end{tabular}

Source: Authors' calculations using HealthFacts RI.

Notes: Robust standard errors in parentheses. In columns 1 and 3, the dependent variable is an indicator of receiving methadone treatment or a buprenorphine prescription, respectively, in at least one month. In columns 2 and 4, the dependent variable is an indicator of receiving methadone treatment or a buprenorphine prescription in at least three months. "Incumbent sample" is shorthand for the opioid dependence sample within the restricted incumbent sample, described in the Notes to Table 3. "Basic sample" is shorthand for a larger sample consisting of all Rhode Island residents at least 18 years of age, not insured by United Healthcare, with at least six consecutive monthly observations, and who had an opioid dependence diagnosis at least once. Both samples further exclude observations with an unknown medical insurance carrier and individuals with missing poverty indicators in more than half of their observations. In columns 1 and 3, Medicaid status is defined as of an individual's earliest observation in 2013, which agrees with the same individual's Medicaid status in most of their other observations. In columns 2 and 4, Medicaid status is defined as having Medicaid insurance in most of one's observations. Medicaid does not include those who hold both Medicare and Medicaid insurance simultaneously. See Notes to Table 4 for definitions of "initial age," "alcohol use disorder (ever)," and "high-poverty Zip code." 
Table A3: Probit Models of the Probability of Having at Least Three Months of Methadone/Buprenorphine Treatment Including Indicators of Pregnancy and Benzodiazepine Receipt

\begin{tabular}{|c|c|c|c|c|}
\hline & \multicolumn{2}{|r|}{ Methadone } & \multicolumn{2}{|c|}{ Buprenorphine } \\
\hline & (1) & (2) & (3) & (4) \\
\hline & All & Females Ages 18-44 & All & Females Ages 18-44 \\
\hline Initial Age 45-64 & $\begin{array}{c}-0.314^{* * *} \\
(0.038)\end{array}$ & & $\begin{array}{c}-0.362^{* * *} \\
(0.032)\end{array}$ & \\
\hline Initial Age 65+ & $\begin{array}{c}-0.837^{* * *} \\
(0.116)\end{array}$ & & $\begin{array}{c}-1.010^{* * *} \\
(0.086)\end{array}$ & \\
\hline Medicaid & $\begin{array}{c}0.694^{* * *} \\
(0.037)\end{array}$ & $\begin{array}{c}0.669^{* * *} \\
(0.067)\end{array}$ & $\begin{array}{c}0.030 \\
(0.034)\end{array}$ & $\begin{array}{c}0.211^{\text {*** }} \\
(0.059)\end{array}$ \\
\hline Female, Never Pregnant & $\begin{array}{c}-0.101^{* * *} \\
(0.038)\end{array}$ & & $\begin{array}{c}-0.146^{* * *} \\
(0.033)\end{array}$ & \\
\hline Female, Ever Pregnant & $\begin{array}{c}0.008 \\
(0.059)\end{array}$ & & $\begin{array}{l}-0.027 \\
(0.053)\end{array}$ & \\
\hline Pregnant (Ever) & & $\begin{array}{c}0.081 \\
(0.065)\end{array}$ & & $\begin{array}{c}0.090 \\
(0.060)\end{array}$ \\
\hline Alcohol Use Disorder (Ever) & $\begin{array}{c}-0.379^{* * *} \\
(0.037)\end{array}$ & $\begin{array}{c}-0.321^{* * *} \\
(0.067)\end{array}$ & $\begin{array}{l}-0.003 \\
(0.031)\end{array}$ & $\begin{array}{l}-0.007 \\
(0.060)\end{array}$ \\
\hline Opioid Overdose (Ever) & $\begin{array}{c}0.322^{* * *} \\
(0.052)\end{array}$ & $\begin{array}{c}0.403^{* * *} \\
(0.097)\end{array}$ & $\begin{array}{c}0.245^{* * *} \\
(0.046)\end{array}$ & $\begin{array}{c}0.345^{* * *} \\
(0.092)\end{array}$ \\
\hline Hepatitis C (Ever) & $\begin{array}{c}0.963^{* * *} \\
(0.041)\end{array}$ & $\begin{array}{c}0.977^{* * *} \\
(0.084)\end{array}$ & $\begin{array}{c}0.217^{* * *} \\
(0.038)\end{array}$ & $\begin{array}{c}0.326^{* * *} \\
(0.081)\end{array}$ \\
\hline Depression (Ever) & $\begin{array}{c}0.014 \\
(0.050)\end{array}$ & $\begin{array}{c}0.025 \\
(0.103)\end{array}$ & $\begin{array}{l}-0.024 \\
(0.042)\end{array}$ & $\begin{array}{l}-0.079 \\
(0.095)\end{array}$ \\
\hline Anxiety (Ever) & $\begin{array}{l}-0.062 \\
(0.050)\end{array}$ & $\begin{array}{l}-0.093 \\
(0.109)\end{array}$ & $\begin{array}{c}0.008 \\
(0.044)\end{array}$ & $\begin{array}{l}-0.030 \\
(0.100)\end{array}$ \\
\hline Other Mental Illness (Ever) & $\begin{array}{c}0.034 \\
(0.056)\end{array}$ & $\begin{array}{c}0.063 \\
(0.120)\end{array}$ & $\begin{array}{l}-0.021 \\
(0.048)\end{array}$ & $\begin{array}{c}0.059 \\
(0.111)\end{array}$ \\
\hline Other Substance Use Disorder (Ever) & $\begin{array}{c}0.235^{* * *} \\
(0.046)\end{array}$ & $\begin{array}{c}0.326^{* * *} \\
(0.089)\end{array}$ & $\begin{array}{c}0.502^{* * *} \\
(0.041)\end{array}$ & $\begin{array}{c}0.536^{* * *} \\
(0.084)\end{array}$ \\
\hline High-poverty Zip Code & $\begin{array}{c}0.111^{* * *} \\
(0.040)\end{array}$ & $\begin{array}{c}0.082 \\
(0.069)\end{array}$ & $\begin{array}{c}-0.174^{* * *} \\
(0.036)\end{array}$ & $\begin{array}{c}-0.281^{* * *} \\
(0.066)\end{array}$ \\
\hline Received Benzodiazepines (Ever) & $\begin{array}{l}-0.008 \\
(0.037)\end{array}$ & $\begin{array}{l}-0.003 \\
(0.070)\end{array}$ & $\begin{array}{c}0.135^{* * *} \\
(0.033)\end{array}$ & $\begin{array}{l}-0.022 \\
(0.064)\end{array}$ \\
\hline Constant & $\begin{array}{c}-1.266^{* * *} \\
(0.061)\end{array}$ & $\begin{array}{c}-1.432^{* * *} \\
(0.138)\end{array}$ & $\begin{array}{c}-0.797^{* * *} \\
(0.053)\end{array}$ & $\begin{array}{c}-0.936^{* * *} \\
(0.119)\end{array}$ \\
\hline Observations & 8951 & 2262 & 8951 & 2262 \\
\hline
\end{tabular}

Source: Authors' calculations using HealthFacts RI.

Notes: Robust standard errors in parentheses. "All" is shorthand for the opioid dependence sample within the restricted incumbent sample, described in the Notes to Table 3. In columns 2 and 4, this sample is further restricted to females who were 18 to 44 years old as of their first observation in the analysis sample. See Notes to Table 4 for definitions of "initial age," "alcohol use disorder (ever)," and "high-poverty Zip code." 
Table A4: Probit Models of the Probability of Having at Least Three Months of Methadone/Buprenorphine Treatment Including Interactions between Age Group and Medicaid Status

$(1)$

(2)

Methadone (At Least 3 Months) Buprenorphine (At Least 3 Months)

\begin{tabular}{|c|c|c|}
\hline 64 & $\begin{array}{c}-0.359^{* * *} \\
(0.068)\end{array}$ & $\begin{array}{c}-0.358^{* * *} \\
(0.044)\end{array}$ \\
\hline 90 & $\begin{array}{c}-0.918^{* * *} \\
(0.202)\end{array}$ & $\begin{array}{c}-1.175^{\text {*** }} \\
(0.111)\end{array}$ \\
\hline Medicaid & $\begin{array}{c}0.961^{* * *} \\
(0.053)\end{array}$ & $\begin{array}{l}-0.038 \\
(0.041)\end{array}$ \\
\hline $64 \times$ Medicaid & $\begin{array}{c}-0.077 \\
(0.079)\end{array}$ & $\begin{array}{l}-0.047 \\
(0.061)\end{array}$ \\
\hline $90 \times$ Medicaid & $\begin{array}{l}-0.258 \\
(0.247)\end{array}$ & $\begin{array}{c}0.362^{* *} \\
(0.175)\end{array}$ \\
\hline Female & $\begin{array}{c}-0.104^{* * *} \\
(0.036)\end{array}$ & $\begin{array}{c}-0.096^{* * *} \\
(0.030)\end{array}$ \\
\hline Alcohol Use Disorder (Ever) & $\begin{array}{c}-0.373^{* * *} \\
(0.038)\end{array}$ & $\begin{array}{l}-0.004 \\
(0.031)\end{array}$ \\
\hline Opioid Overdose (Ever) & $\begin{array}{c}0.319^{* * *} \\
(0.052)\end{array}$ & $\begin{array}{c}0.252^{* * *} \\
(0.046)\end{array}$ \\
\hline Hepatitis C (Ever) & $\begin{array}{c}0.885^{* * *} \\
(0.042)\end{array}$ & $\begin{array}{c}0.234^{* * *} \\
(0.039)\end{array}$ \\
\hline Depression (Ever) & $\begin{array}{l}-0.042 \\
(0.052)\end{array}$ & $\begin{array}{l}-0.005 \\
(0.042)\end{array}$ \\
\hline Anxiety (Ever) & $\begin{array}{c}-0.112^{* *} \\
(0.050)\end{array}$ & $\begin{array}{c}0.054 \\
(0.043)\end{array}$ \\
\hline Other Mental Illness (Ever) & $\begin{array}{l}-0.040 \\
(0.058)\end{array}$ & $\begin{array}{l}-0.006 \\
(0.048)\end{array}$ \\
\hline Other Substance Use Disorder (Ever) & $\begin{array}{c}0.153^{* * *} \\
(0.048)\end{array}$ & $\begin{array}{c}0.524^{* * *} \\
(0.041)\end{array}$ \\
\hline High-poverty Zip Code & $\begin{array}{c}0.039 \\
(0.039)\end{array}$ & $\begin{array}{c}-0.161^{* * *} \\
(0.037)\end{array}$ \\
\hline Constant & $\begin{array}{c}-1.314^{* * *} \\
(0.067)\end{array}$ & $\begin{array}{c}-0.774^{* * *} \\
(0.054)\end{array}$ \\
\hline Observations & 8951 & 8951 \\
\hline
\end{tabular}

Source: Authors' calculations using HealthFacts RI.

Notes: Robust standard errors in parentheses. See Notes to Table 3 for a description of the opioid dependence sample within the restricted incumbent panel. Health insurance status is as of January 2013. The Medicaid category includes those who carry both Medicare and Medicaid insurance (dual eligibles). The omitted category is non-Medicaid insurance, and the omitted initial age group is 18 to 44 . See Notes to Table 4 for definitions of "initial age," "alcohol use disorder (ever)," and "highpoverty Zip code." 
Figure A1: Predicted Probability of Receiving Methadone Treatment

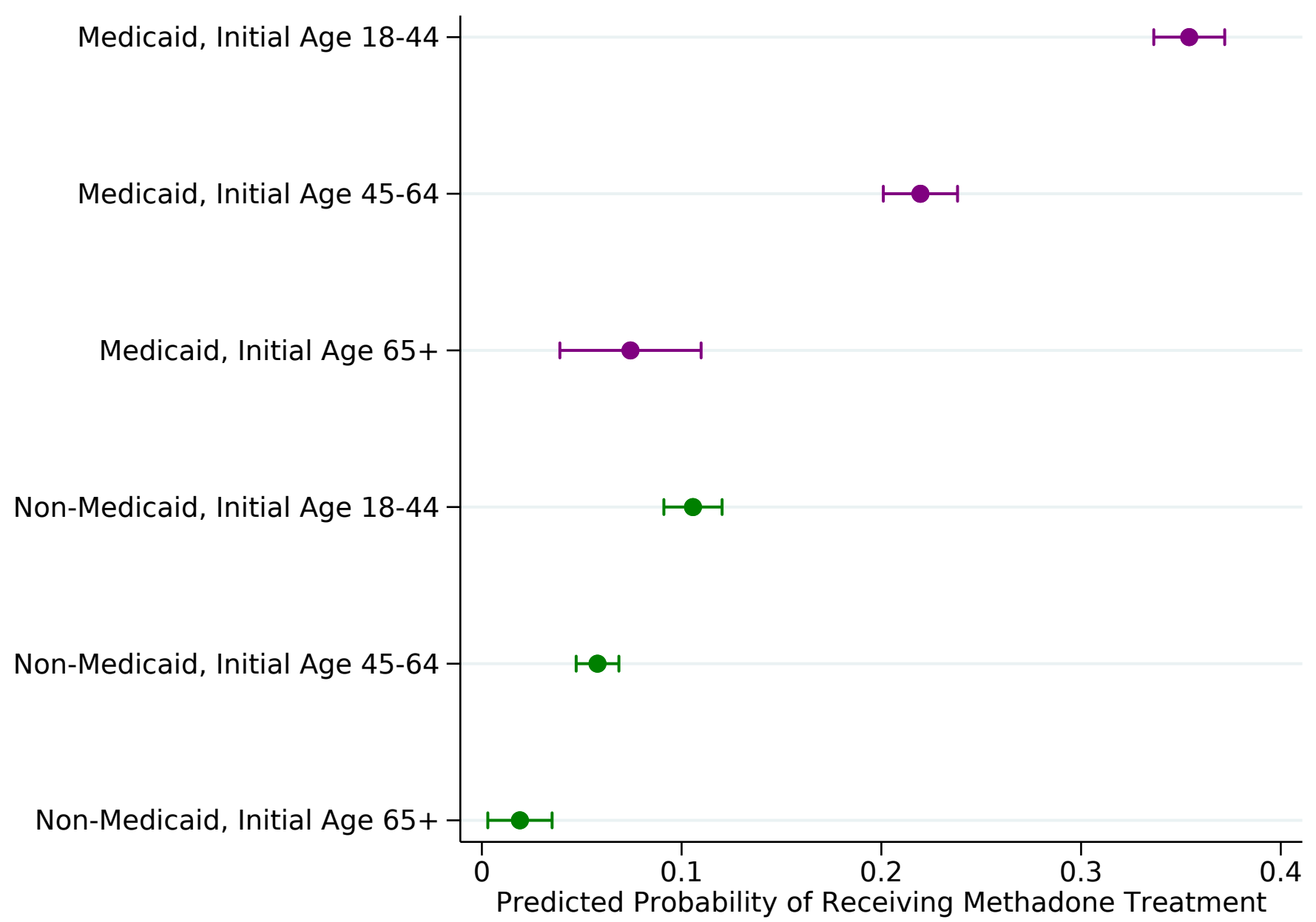

Source: Authors' calculations using HealthFacts RI.

Notes: Probabilities are based on the multivariate probit model of receiving methadone treatment in at least three separate months, shown in Table A4. Sample consists of patients in the restricted incumbent panel who were diagnosed with opioid dependence, see Notes to Tables 3 and 4 for additional details. The Medicaid category includes those who carry both Medicare and Medicaid insurance (dual eligibles). 
Table A5: Probit Models of the Probability of Having at Least Three Months of Buprenorphine/Methadone Treatment, with Rural Indicator

\begin{tabular}{|c|c|c|}
\hline & $\begin{array}{c}\text { (1) } \\
\text { Methadone (At Least } 3 \text { Months) }\end{array}$ & $\begin{array}{c}(2) \\
\text { Buprenorphine (At Least } 3 \text { Months) }\end{array}$ \\
\hline Initial Age 45-64 & $\begin{array}{l}-0.329^{* * *} \\
(0.037)\end{array}$ & $\begin{array}{c}-0.367^{* * *} \\
(0.032)\end{array}$ \\
\hline Initial Age 65+ & $\begin{array}{c}-0.851^{* * *} \\
(0.116)\end{array}$ & $\begin{array}{c}-1.019^{* * *} \\
(0.086)\end{array}$ \\
\hline Medicaid & $\begin{array}{c}0.701^{* * *} \\
(0.037)\end{array}$ & $\begin{array}{c}0.033 \\
(0.033)\end{array}$ \\
\hline Female & $\begin{array}{c}-0.079^{* *} \\
(0.036)\end{array}$ & $\begin{array}{l}-0.107^{* * *} \\
(0.030)\end{array}$ \\
\hline Alcohol Use Disorder (Ever) & $\begin{array}{c}-0.379^{* * *} \\
(0.037)\end{array}$ & $\begin{array}{l}-0.004 \\
(0.031)\end{array}$ \\
\hline Opioid Overdose (Ever) & $\begin{array}{c}0.321^{* * *} \\
(0.052)\end{array}$ & $\begin{array}{c}0.249^{* * *} \\
(0.046)\end{array}$ \\
\hline Hepatitis C (Ever) & $\begin{array}{c}0.962^{* * *} \\
(0.041)\end{array}$ & $\begin{array}{l}0.214^{* * *} \\
(0.038)\end{array}$ \\
\hline Depression (Ever) & $\begin{array}{c}0.014 \\
(0.050)\end{array}$ & $\begin{array}{l}-0.010 \\
(0.042)\end{array}$ \\
\hline Anxiety (Ever) & $\begin{array}{l}-0.067 \\
(0.049)\end{array}$ & $\begin{array}{c}0.054 \\
(0.043)\end{array}$ \\
\hline Other Mental Illness (Ever) & $\begin{array}{c}0.035 \\
(0.056)\end{array}$ & $\begin{array}{l}-0.008 \\
(0.048)\end{array}$ \\
\hline Other Substance Use Disorder (Ever) & $\begin{array}{c}0.239^{* * *} \\
(0.046)\end{array}$ & $\begin{array}{l}0.510^{* * *} \\
(0.040)\end{array}$ \\
\hline High-poverty Zip Code & $\begin{array}{l}0.109^{* * *} \\
(0.040)\end{array}$ & $\begin{array}{c}-0.182^{* * *} \\
(0.037)\end{array}$ \\
\hline Rural Zip Code & $\begin{array}{l}-0.021 \\
(0.081)\end{array}$ & $\begin{array}{l}-0.004 \\
(0.066)\end{array}$ \\
\hline Constant & $\begin{array}{l}-1.265^{* * *} \\
\quad(0.062)\end{array}$ & $\begin{array}{l}-0.780^{* * *} \\
(0.053)\end{array}$ \\
\hline Observations & 8951 & 8951 \\
\hline
\end{tabular}

Source: Authors' calculations using HealthFacts RI.

Notes: Robust standard errors in parentheses. See Notes to Table 3 for a description of the opioid dependence sample within the restricted incumbent panel. "Rural Zip code" indicates that the individual lived in a rural Zip code in the majority of their observations. A rural Zip code is one in which fewer than 50 percent of residents live in a city or town with a population of at least 50,000. See Notes to Table 4 for definitions of "initial age," "alcohol use disorder (ever)," and "high-poverty Zip code." 


\section{List of Diagnosis, Procedure, and Drug Codes}

\section{ICD-9 and ICD-10 Diagnosis Codes}

Descriptions follow tables of numerical codes.

Opioid Use Disorder (OUD)

\begin{tabular}{|l|l|l|l|l|l|l|l|}
\hline 30400 & 30553 & F11220 & F11251 & F1111 & F11151 & F11920 & F11951 \\
\hline 30401 & 30470 & F11221 & F11259 & F11120 & F11159 & F11921 & F11959 \\
\hline 30402 & 30471 & F11222 & F11281 & F11121 & F11181 & F11922 & F11981 \\
\hline 30403 & 30472 & F11229 & F11282 & F11122 & F11182 & F11929 & F11982 \\
\hline 30550 & 30473 & F1123 & F11288 & F11129 & F11188 & F1193 & F11988 \\
\hline 30551 & F1120 & F1124 & F1129 & F1114 & F1119 & F1194 & F1199 \\
\hline 30552 & F1121 & F11250 & F1110 & F11150 & F1190 & F11950 & \\
\hline
\end{tabular}

Opioid Overdose

\begin{tabular}{|c|c|c|c|c|c|c|}
\hline 96500 & E8501 & T400X4A & T402X1A & T403X2A & T404X3A & T40604A \\
\hline 96501 & E8502 & T401X1A & T402X2A & T403X3A & T404X4A & T40691A \\
\hline 96502 & T400X1A & T401X2A & T402X3A & T403X4A & T40601A & T40692A \\
\hline 96509 & T400X2A & T401X3A & T402X4A & T404X1A & T40602A & T40693A \\
\hline E8500 & T400X3A & T401X4A & T403X1A & T404X2A & T40603A & T40694A \\
\hline
\end{tabular}

$\underline{\text { Alcohol Use Disorder (AUD) }}$

\begin{tabular}{|l|l|l|l|l|l|l|l|}
\hline 30300 & 30392 & F1010 & F10150 & F10188 & F10229 & F10250 & F10281 \\
\hline 30301 & 30393 & F1011 & F10151 & F1019 & F10230 & F10251 & F10282 \\
\hline 30302 & 30500 & F10120 & F10159 & F1020 & F10231 & F10259 & F10288 \\
\hline 30303 & 30501 & F10121 & F10180 & F1021 & F10232 & F1026 & F1029 \\
\hline 30390 & 30502 & F10129 & F10181 & F10220 & F10239 & F1027 & \\
\hline 30391 & 30503 & F1014 & F10182 & F10221 & F1024 & F10280 & \\
\hline
\end{tabular}

Hepatitis C

\begin{tabular}{|c|c|c|c|c|c|}
\hline 07041 & 07051 & 07070 & B1710 & B182 & B1921 \\
\hline 07044 & 07054 & 07071 & B1711 & B1920 & \\
\hline
\end{tabular}

Anxiety

\begin{tabular}{|c|c|c|c|c|c|c|c|}
\hline 3009 & 30023 & 3130 & 3005 & F40240 & F419 & F4310 & R455 \\
\hline 30000 & 29384 & 30001 & F064 & F40298 & F42 & F4311 & R456 \\
\hline 30981 & 3089 & 3082 & F4000 & F408 & F422 & F4312 & R4581 \\
\hline 30021 & 3083 & 31322 & F4001 & F409 & F423 & F488 & \\
\hline 30002 & 30009 & 31383 & F4002 & F410 & F424 & F489 & \\
\hline 3003 & 3133 & 30020 & F4010 & F411 & F428 & R452 & \\
\hline 30029 & 30010 & 30022 & F4011 & F413 & F429 & R453 & \\
\hline 3080 & 30089 & 3081 & F40232 & F418 & F430 & R454 & \\
\hline
\end{tabular}


Depression

\begin{tabular}{|l|l|l|l|l|l|l|l|}
\hline 29383 & 29624 & 29632 & 3004 & F323 & F3289 & F333 & F339 \\
\hline 29620 & 29625 & 29633 & 311 & F324 & F329 & F3340 & F341 \\
\hline 29621 & 29626 & 29634 & F320 & F328 & F330 & F3341 & \\
\hline 29622 & 29630 & 29635 & F321 & F328 & F331 & F3342 & \\
\hline 29623 & 29631 & 29636 & F322 & F3281 & F332 & F338 & \\
\hline
\end{tabular}

Other Substance Use Disorder (SUD)

\begin{tabular}{|c|c|c|c|c|c|c|c|}
\hline 2920 & 30463 & 30590 & F1299 & F1420 & F1529 & F18951 & F19959 \\
\hline 29211 & 30470 & 30591 & F1310 & F1421 & F1590 & F1910 & F19980 \\
\hline 29212 & 30471 & 30592 & F1311 & F14220 & F15921 & F1911 & F19982 \\
\hline 2922 & 30472 & 30593 & F13120 & F14229 & F15922 & F19120 & F1999 \\
\hline 29281 & 30473 & 64830 & F13121 & F1423 & F1594 & F19121 & F558 \\
\hline 29283 & 30480 & 64831 & F13129 & F1424 & F15950 & F19129 & O355XX0 \\
\hline 29284 & 30481 & 64833 & F13188 & F14259 & F15951 & F1914 & O99320 \\
\hline 29285 & 30482 & 65553 & F1320 & F14280 & F15959 & F19150 & O99321 \\
\hline 29289 & 30483 & 76072 & F1321 & F14288 & F1599 & F19159 & O99322 \\
\hline 2929 & 30490 & F1210 & F13220 & F1429 & F1610 & F19188 & O99323 \\
\hline 30410 & 30491 & F1211 & F13221 & F1490 & F1611 & F1920 & O99324 \\
\hline 30411 & 30492 & F12120 & F13230 & F14921 & F16121 & F1921 & O99325 \\
\hline 30420 & 30493 & F12122 & F13231 & F14922 & F1614 & F19221 & T401X1D \\
\hline 30421 & 30520 & F12129 & F13232 & F14929 & F16151 & F19229 & T401X1S \\
\hline 30422 & 30521 & F12150 & F13239 & F1494 & F1620 & F19230 & T405X1A \\
\hline 30423 & 30522 & F12151 & F1390 & F14959 & F1621 & F19231 & T405X4A \\
\hline 30430 & 30523 & F12180 & F1394 & F1499 & F16951 & F19239 & T405X5A \\
\hline 30431 & 30530 & F12188 & F13980 & F1510 & F16959 & F1924 & T407X1A \\
\hline 30432 & 30540 & F1220 & F1399 & F15120 & $\mathrm{F} 17200$ & F19251 & T407X4A \\
\hline 30433 & 30541 & F1221 & F1410 & F15122 & F17203 & F1929 & T407X5A \\
\hline 30440 & 30543 & F12229 & F1411 & F15150 & F17208 & F1990 & T408X1A \\
\hline 30441 & 30560 & $\mathrm{~F} 12250$ & F14120 & F15159 & F17209 & F19920 & T408X4A \\
\hline 30443 & 30561 & F12288 & F14122 & F1519 & F17210 & F19921 & T40991A \\
\hline 30450 & 30562 & F1229 & F14129 & F1520 & F17213 & F19929 & \\
\hline 30451 & 30563 & F1290 & F1414 & F1521 & F17223 & F19931 & \\
\hline 30453 & 30570 & F12920 & F14159 & F15220 & F17290 & F19939 & \\
\hline 30460 & 30571 & F12921 & F14180 & F1523 & F1810 & F1994 & \\
\hline 30461 & 30572 & F12929 & F14188 & F1524 & F18159 & F19950 & \\
\hline 30462 & 30580 & F12988 & F1419 & F15259 & F1820 & F19951 & \\
\hline
\end{tabular}


Other Mental Illness

\begin{tabular}{|c|c|c|c|c|c|c|c|c|c|}
\hline 2900 & 29571 & 29664 & 30182 & 30923 & F0631 & F3173 & F5105 & F939 & T43212A \\
\hline 29010 & 29572 & 29665 & 30183 & 30924 & F0632 & F3174 & F5109 & F941 & T43222A \\
\hline 29011 & 29573 & 29666 & 30189 & 30928 & F0633 & F3177 & F5113 & F952 & T43222S \\
\hline 29021 & 29574 & 2967 & 3019 & 30929 & F0634 & F3181 & F514 & F959 & T43292A \\
\hline 2903 & 29575 & 29681 & 3022 & 3093 & F068 & F3189 & F515 & F980 & T433X2A \\
\hline 29040 & 29580 & 29690 & 30250 & 3094 & F070 & F319 & F519 & F984 & T43502A \\
\hline 2909 & 29582 & 29699 & 3026 & 30982 & F0781 & F3281 & F520 & F988 & T43592A \\
\hline 2930 & 29584 & 2970 & 30270 & 30983 & F09 & F3289 & F5221 & F989 & T43612A \\
\hline 2931 & 29590 & 2971 & 30272 & 30989 & F200 & F338 & F524 & F99 & T43622A \\
\hline 29381 & 29591 & 2978 & 30275 & 3099 & F201 & F340 & F53 & G301 & T438X2A \\
\hline 29382 & 29592 & 2979 & 30281 & 3100 & F202 & F341 & F600 & G309 & T446X2A \\
\hline 29383 & 29594 & 2981 & 30285 & 3101 & F203 & F348 & F601 & G311 & T447X2A \\
\hline 29389 & 29595 & 2982 & 3029 & 3102 & F205 & F3481 & F602 & G3183 & $\mathrm{T} 450 \mathrm{X} 2 \mathrm{~A}$ \\
\hline 2939 & 29600 & 2983 & 3051 & 31230 & F2081 & F3489 & F603 & R4181 & $\mathrm{T} 452 \mathrm{X} 2 \mathrm{~A}$ \\
\hline 2940 & 29601 & 2988 & 3061 & 31231 & F2089 & F349 & F604 & R45850 & T45512A \\
\hline 29410 & 29602 & 2989 & 3063 & 31232 & F209 & F39 & F605 & R45851 & T461X2A \\
\hline 29411 & 29603 & 29900 & 3064 & 31234 & F22 & F440 & F607 & R4586 & T463X2A \\
\hline 29420 & 29604 & 29910 & 30652 & 31239 & F23 & F444 & F6081 & R4589 & T465X2A \\
\hline 29421 & 29605 & 29911 & 30653 & 31323 & F24 & F445 & F6089 & R780 & T481X2A \\
\hline 2948 & 29606 & 29980 & 3069 & 3139 & F250 & F447 & F609 & R781 & T483X2A \\
\hline 2949 & 29610 & 29990 & 3071 & 31389 & F258 & F4481 & F630 & R784 & T484X2A \\
\hline 29500 & 29612 & 30011 & 30720 & 316 & F259 & F449 & F632 & R785 & T490X2A \\
\hline 29502 & 29613 & 30013 & 30721 & 3310 & F28 & F450 & F633 & T381X2A & T507X2A \\
\hline 29503 & 29615 & 30014 & 30722 & 33111 & F29 & F451 & F6381 & T383X2A & T508X2A \\
\hline 29510 & 29630 & 30015 & 30723 & 3312 & F3010 & F4520 & F6389 & T39012A & T50902A \\
\hline 29512 & 29631 & 30016 & 30740 & 33182 & F3012 & F4521 & F639 & T391X2A & T50902D \\
\hline 29514 & 29632 & 30081 & 30741 & 33392 & F302 & F4522 & F640 & T39312A & T50992A \\
\hline 29520 & 29633 & 30082 & 30742 & 64840 & F308 & F4541 & F641 & T39392A & T510X2A \\
\hline 29525 & 29634 & 3006 & 30743 & 64841 & F310 & F4542 & F649 & T398X2A & T518X2A \\
\hline 29530 & 29635 & 3007 & 30745 & 64843 & F3110 & F458 & F654 & T401X2S & T5192XA \\
\hline 29531 & 29636 & 3010 & 30746 & 64844 & F3111 & F459 & F659 & T405X2A & T524X2A \\
\hline 29532 & 29640 & 30110 & 30747 & 797 & F3112 & F481 & F66 & T4142XA & T528X2A \\
\hline 29533 & 29641 & 30112 & 30750 & F0150 & F3113 & F5002 & F6810 & T420X2A & T5292XA \\
\hline 29534 & 29642 & 30113 & 30751 & F0151 & F312 & F502 & F6811 & T421X2A & T5802XA \\
\hline 29535 & 29643 & 30120 & 30752 & F0280 & F3160 & F508 & F688 & T424X2A & T5892XA \\
\hline 29540 & 29644 & 30122 & 30754 & F0281 & F3161 & F5081 & F69 & T426X2A & T65222D \\
\hline 29544 & 29645 & 3013 & 30759 & F0390 & F3162 & F5089 & F840 & T4272XA & T6592XA \\
\hline 29552 & 29646 & 3014 & 3076 & F0391 & F3163 & F509 & F845 & T428X2A & T71162A \\
\hline 29560 & 29660 & 30150 & 30780 & F05 & F3164 & F5101 & F848 & T43012A & \\
\hline 29562 & 29661 & 3016 & 30781 & F060 & F3170 & F5102 & F849 & T43012S & \\
\hline 29564 & 29662 & 3017 & 30789 & F062 & F3171 & F5103 & F930 & $\mathrm{T} 43022 \mathrm{~A}$ & \\
\hline 29570 & 29663 & 30181 & 30921 & F0630 & F3172 & F5104 & F938 & $\mathrm{T} 43202 \mathrm{~A}$ & \\
\hline
\end{tabular}


Pregnancy (ICD-9/10 and CPT Codes)

\begin{tabular}{|c|c|c|c|c|c|c|c|}
\hline V39 & V235 & 64844 & Z370 & O09813 & O133 & O360110 & O4412 \\
\hline V30 & V235 & 64630 & Z372 & O09819 & O139 & O360120 & O4412 \\
\hline V289 & V2349 & 64510 & Z373 & O09891 & O24419 & O360130 & O4413 \\
\hline V283 & V2342 & 64243 & Z3750 & O09892 & O1400 & O360190 & O480 \\
\hline V282 & V2341 & 64240 & Z3751 & O09893 & O1402 & O365910 & O001 \\
\hline V280 & V232 & 64233 & Z371 & O09899 & O1403 & O365920 & O009 \\
\hline V2631 & V231 & 64230 & Z374 & Z3400 & O2620 & O365930 & O019 \\
\hline V2629 & V230 & 64113 & Z377 & Z3401 & O2621 & O365990 & O020 \\
\hline V242 & V222 & 64100 & O09511 & Z3402 & O2622 & O3660X0 & O021 \\
\hline V239 & V221 & 64000 & O09512 & Z3403 & O2623 & O3661X0 & O039 \\
\hline V2389 & V220 & 64844 & O09513 & Z3490 & O26851 & O3662X0 & O200 \\
\hline V2387 & 65660 & 63790 & O09519 & Z3491 & O26852 & O3663X0 & O364XX0 \\
\hline V2386 & 65650 & 63490 & O09521 & Z3492 & O26853 & O4400 & O99345 \\
\hline V2384 & 65640 & 63390 & O09522 & Z3493 & O26859 & O4401 & \\
\hline V2383 & 65610 & 63310 & O09523 & Z36 & O30001 & O4402 & CPT: \\
\hline V2382 & 65100 & 63200 & O09529 & Z392 & O30002 & O4403 & 59400 \\
\hline V2381 & 64950 & 63180 & O09811 & O131 & O30003 & O4410 & 59510 \\
\hline V237 & 64880 & 630 & O09812 & O132 & O30009 & O4411 & 59610 \\
\hline
\end{tabular}

\section{CPT/HCPCS Procedure Codes}

Methadone Maintenance Treatment

\section{$\mathrm{H} 0020$}

Behavioral Therapy

\begin{tabular}{|l|l|l|l|l|l|}
\hline 90832 & 90836 & 90846 & 90853 & 99411 & H0005 \\
\hline 90833 & 90837 & 90847 & 90875 & 99412 & T1006 \\
\hline 90834 & 90838 & 90849 & 90876 & H0004 & T1012 \\
\hline
\end{tabular}




\section{National Drug Codes}

\section{Buprenorphine}

Buprenorphine National Drug Codes were pulled from two data sources:

1) US Food and Drug Administration's National Drug Code Directory (updated daily).

Drugs were pulled on a text search for buprenorphine/

buprenorphine-naloxone combinations and manually reviewed.

2) 2018 CDC Opioid National Drug Code (NDC) and Oral Morphine Milligram

Equivalent (MME) Conversion File.

The following formulations were excluded based on their proprietary name: Buprenex, Butrans, and Belbuca. Non-proprietary (generic) versions of these formulations were excluded, based on the following national drug codes.

Buprenex (generic):

\begin{tabular}{|l|l|l|l|}
\hline 00517072505 & 00517072501 & 00409201232 & 42023017905 \\
\hline
\end{tabular}

Butrans (generic):

\begin{tabular}{|l|l|l|l|}
\hline 00093365640 & 00093365740 & 00093365840 & 00093365940 \\
\hline
\end{tabular}

All buprenorphine formulations with the dosage form field set to "solution" or "patch, extended release" were excluded.

\section{Opioids}

Opioid National Drug Codes were pulled from the 2018 CDC Opioid NDC and Oral MME Conversion File.

\section{Benzodiazepines}

Benzodiazepine National Drug Codes were pulled from the 2018 CDC Opioid NDC and Oral MME Conversion File. 